TRANSACTIONS OF THE

AMERICAN MATHEMATICAL SOCIETY

Volume 360, Number 7, July 2008, Pages 3645-3686

S 0002-9947(08)04341-9

Article electronically published on February 13, 2008

\title{
AFFINE GEOMETRIC CRYSTALS AND LIMIT OF PERFECT CRYSTALS
}

\author{
MASAKI KASHIWARA, TOSHIKI NAKASHIMA, AND MASATO OKADO
}

\begin{abstract}
For every non-exceptional affine Lie algebra, we explicitly construct a positive geometric crystal associated with a fundamental representation. We also show that its ultra-discretization is isomorphic to the limit of certain perfect crystals of the Langlands dual affine Lie algebra.
\end{abstract}

\section{INTRODUCTION}

The theory of perfect crystals was introduced in [7, 8 in order to study certain physical models, called vertex models, which are associated with quantum $R$ matrices. A perfect crystal is a (pseudo-)crystal base of a finite-dimensional module of a quantum affine algebra $U_{q}(\mathfrak{g})$ (for more details, see 3.3), and it is labeled by a positive integer $l$, called the level (Definition 3.6). One of the most important properties of perfect crystals is as follows: for a perfect crystal $B$ of level $l$ and the crystal $B(\lambda)$ of the irreducible $U_{q}(\mathfrak{g})$-module with a dominant integral weight $\lambda$ of level $l$ as a highest weight, there exist a unique dominant integral weight $\mu$ of level $l$ and an isomorphism of crystals:

$$
B(\lambda) \cong B(\mu) \otimes B
$$

By iterating this isomorphism, we obtain the path realization of $B(\lambda)$, which plays a crucial role in solving the vertex models. In 8 , for each $\mathfrak{g}=A_{n}^{(1)}, B_{n}^{(1)}, C_{n}^{(1)}$, $D_{n}^{(1)}, A_{2 n-1}^{(2)}, D_{n+1}^{(2)}, A_{2 n}^{(2)}$, perfect crystals are constructed explicitly by patching two classical crystals. It is conjectured that certain "Kirillov-Reshetikhin modules" have perfect crystal bases and all perfect crystals are obtained as a tensor product of those perfect crystal bases ([2], 3]), but, it is still far from the complete classification of perfect crystals.

Let $\left\{B_{l}\right\}_{l \geq 1}$ be a family of perfect crystals. If it satisfies certain conditions (Definition [3.7), there exists a limit $B_{\infty}$ of $\left\{B_{l}\right\}_{l \geq 1}$. In such a case the family $\left\{B_{l}\right\}_{l \geq 1}$ is called a coherent family of perfect crystals $(\underline{6})$. Let $B(\infty)$ be the crystal of the lower nilpotent subalgebras $U_{q}^{-}(\mathfrak{g})$ of the quantum algebra $U_{q}(\mathfrak{g})$. Then, similarly to perfect crystals, we have an isomorphism of crystals:

$$
B(\infty) \otimes B_{\infty} \cong B(\infty)
$$

Received by the editors December 29, 2005 and, in revised form, May 11, 2006.

2000 Mathematics Subject Classification. Primary 17B37, 17B67; Secondary 22E65, 14M15. 
By iterating this isomorphism, the path realization of $B(\infty)$ is obtained. In [6], for $\mathfrak{g}=A_{n}^{(1)}, B_{n}^{(1)}, C_{n}^{(1)}, D_{n}^{(1)}, A_{2 n-1}^{(2)}, D_{n+1}^{(2)}, A_{2 n}^{(2)}$, explicit forms of the limit of perfect crystals are described, which are also reviewed in $\oint 3$ below.

A. Berenstein and D. Kazhdan introduced the theory of geometric crystals ([1]), which is a structure on geometric objects analogous to crystals. Let $I$ be the index set of simple roots of $\mathfrak{g}$. A geometric crystal consists of a variety $X$, a rational $\mathbb{C}^{\times}$- action $e_{i}: \mathbb{C}^{\times} \times X \longrightarrow X$ and rational functions $\gamma_{i}, \varepsilon_{i}: X \longrightarrow \mathbb{C}$ which satisfy certain conditions (see Definition 2.1). This structure resembles the one of crystals; for instance, we have the tensor product of a pair of geometric crystals similarly to the tensor product of crystals. There is a more direct and remarkable relation between crystals and geometric crystals, that is, the ultra-discretization functor $\mathcal{U D}$. This is a functor from the category of positive geometric crystals to that of crystals, where a positive geometric crystal is a geometric crystal equipped with a positive structure (see 22.4 ). Applying this functor, positive rational functions are transferred to piecewise linear functions by the following simple correspondence:

$$
x \times y \mapsto x+y, \quad \frac{x}{y} \mapsto x-y, \quad x+y \mapsto \max (x, y) .
$$

The purpose of this paper is to construct a positive affine geometric crystal $\mathcal{V}(\mathfrak{g})$, whose ultra-discretization is isomorphic to a limit of perfect crystals $B_{\infty}\left(\mathfrak{g}^{L}\right)$, where $\mathfrak{g}^{L}$ is the Langlands dual of $\mathfrak{g}$.

Let $G$ (resp. $\mathfrak{g}$ ) be the affine Kac-Moody group (resp. algebra) associated with a generalized Cartan matrix $A=\left(a_{i j}\right)_{i, j \in I}$. Let $B^{ \pm}$be the Borel subgroup and $T$ the maximal torus. Set $y_{i}(c):=\exp \left(c f_{i}\right)$, and let $\alpha_{i}^{\vee}(c) \in T$ be the image of $c \in \mathbb{C}^{\times}$by the group morphism $\mathbb{C}^{\times} \rightarrow T$ induced by the simple coroot $\alpha_{i}^{\vee}$ as in 2.1 We set $Y_{i}(c):=y_{i}\left(c^{-1}\right) \alpha_{i}^{\vee}(c)=\alpha_{i}^{\vee}(c) y_{i}(c)$. Let $W$ (resp. $\widetilde{W}$ ) be the Weyl group (resp. the extended Weyl group) associated with $\mathfrak{g}$. The Schubert cell $X_{w}:=B w B / B\left(w=s_{i_{1}} \cdots s_{i_{k}} \in W\right)$ is isomorphic to the variety

$$
B_{w}^{-}:=\left\{Y_{i_{1}}\left(x_{1}\right) \cdots Y_{i_{k}}\left(x_{k}\right) \mid x_{1}, \cdots, x_{k} \in \mathbb{C}^{\times}\right\} \subset B^{-},
$$

and $X_{w}$ has a natural geometric crystal structure ([1], [14]).

We choose $0 \in I$ as in [9], and let $\left\{\varpi_{i}\right\}_{i \in I \backslash\{0\}}$ be the set of level 0 fundamental weights. Let $W\left(\varpi_{i}\right)$ be the fundamental representation of $U_{q}(\mathfrak{g})$ with $\varpi_{i}$ as an extremal weight $([9])$. Let us denote its reduction at $q=1$ by the same notation $W\left(\varpi_{i}\right)$. It is a finite-dimensional $\mathfrak{g}$-module. The module $W\left(\varpi_{i}\right)$ is irreducible over $U_{q}(\mathfrak{g})$, but its reduction at $q=1$ is not necessarily an irreducible $\mathfrak{g}$-module. We set $\mathbb{P}\left(\varpi_{i}\right):=\left(W\left(\varpi_{i}\right) \backslash\{0\}\right) / \mathbb{C}^{\times}$.

For any $i \in I$, define (see 3.2 for the definition of the inner product),

$$
c_{i}^{\vee}:=\max \left(1, \frac{2}{\left(\alpha_{i}, \alpha_{i}\right)}\right)
$$

Then the translation $t\left(c_{i}^{\vee} \varpi_{i}\right)$ belongs to $\widetilde{W}$ (see $\$ 5.1$ ). For a subset $J$ of $I$, let us denote by $\mathfrak{g}_{J}$ the subalgebra of $\mathfrak{g}$ generated by $\left\{e_{i}, f_{i}\right\}_{i \in J}$. For an integral weight $\mu$, define $I(\mu):=\left\{j \in I \mid\left\langle h_{j}, \mu\right\rangle \geq 0\right\}$.

Then we have the following conjecture $([2,3,9])$. 
Conjecture 1.1. (1) For any $i \in I \backslash\{0\}$ and any positive integer $n$, there exists a finite-dimensional irreducible $U_{q}(\mathfrak{g})$-module $W\left(n \varpi_{i}\right)$ with extremal weight $n \varpi_{i}$, called the "Kirillov-Reshetikhin module".

(2) The module $W\left(n \varpi_{i}\right)$ has a global crystal basis.

(3) Its crystal $B\left(n \varpi_{i}\right)$ is perfect if and only if $n \in c_{i}^{\vee} \mathbb{Z}$, and its level is equal to $n / c_{i}^{\vee}$.

(4) $\left\{B\left(n c_{i}^{\vee} \varpi_{i}\right)\right\}_{n}$ is a coherent family of perfect crystals. We denote by $B_{\infty}\left(\varpi_{i}\right)$ its limit crystal.

Now, let us state our conjecture.

Conjecture 1.2. For any $i \in I \backslash\{0\}$, there exist a unique variety $X$ endowed with a positive $\mathfrak{g}$-geometric crystal structure and a rational mapping $\pi: X \longrightarrow \mathbb{P}\left(\varpi_{i}\right)$ satisfying the following properties:

(1) for an arbitrary extremal vector $u \in W\left(\varpi_{i}\right)_{\mu}$, writing the translation $t\left(c_{i}^{\vee} \mu\right)$ as $\iota w \in \widetilde{W}$ with a Dynkin diagram automorphism $\iota$ and $w=s_{i_{1}} \cdots s_{i_{k}}$ (see \$.1), there exists a birational mapping $\xi: B_{w}^{-} \longrightarrow X$ such that $\xi$

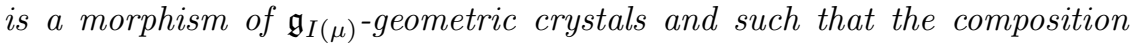
$\pi \circ \xi: B_{w}^{-} \rightarrow \mathbb{P}\left(\varpi_{i}\right)$ coincides with $Y_{i_{1}}\left(x_{1}\right) \cdots Y_{i_{k}}\left(x_{k}\right) \mapsto Y_{i_{1}}\left(x_{1}\right) \cdots Y_{i_{k}}\left(x_{k}\right) \bar{u}$, where $s_{i_{1}} \cdots s_{i_{k}}$ is a reduced expression of $w$ and $\bar{u}$ is the line including $u$,

(2) the ultra-discretization of $X$ is isomorphic to the crystal $B_{\infty}\left(\varpi_{i}\right)$ of the Langlands dual $\mathfrak{g}^{L}$.

In this paper, we construct a positive geometric crystal for some $i=1$ and for $\mathfrak{g}=A_{n}^{(1)}, B_{n}^{(1)}, C_{n}^{(1)}, D_{n}^{(1)}, A_{2 n-1}^{(2)}, D_{n+1}^{(2)}, A_{2 n}^{(2)}$, with this conjecture as a guide.

Let $W\left(\varpi_{1}\right)$ be the fundamental $\mathfrak{g}$-module as above $\left.(9]\right)$ and $u_{1}:=u_{\varpi_{1}}$ an extremal weight vector of $W\left(\varpi_{1}\right)$ with the weight $\varpi_{1}$.

Then $t\left(c_{1}^{\vee} \varpi_{1}\right)$ belongs to $\widetilde{W}$ and (see $\$ 5.1$ ) there exist $w_{1} \in W$ and a Dynkin diagram automorphism $\iota$ such that $t\left(c_{1}^{\vee} \varpi_{1}\right)=\iota \cdot w_{1}$. Associated with this $w_{1}$, we define

$$
X_{1}:=B_{w_{1}}^{-} \text {. }
$$

Then $I\left(\varpi_{1}\right)=I \backslash\{0\}$ and $X_{1}$ has a structure of a $\mathfrak{g}_{I \backslash\{0\}}$-geometric crystal.

Let us choose another extremal weight vector $u_{2}$ with extremal weight $\eta$ such that $I(\eta)=I \backslash\left\{i_{2}\right\}$ for some $i_{2} \neq 0$. Then there exists $w_{2} \in W$ such that $t\left(c_{1}^{\vee} \eta\right)=\iota \cdot w_{2}$. We define similarly

$$
X_{2}:=B_{w_{2}}^{-},
$$

which has a structure of a $\mathfrak{g}_{I \backslash\left\{i_{2}\right\}}$-geometric crystal. We have the rational mapping $X_{\nu} \rightarrow \mathbb{P}\left(\varpi_{1}\right)(\nu=1,2)$.

Then we see (by a case-by-case calculation) that there exists a unique positive birational mapping $\xi: X_{1} \rightarrow X_{2}$ such that the diagram

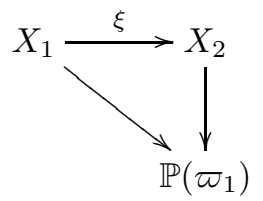

commutes and $\xi$ commutes with $e_{i}$ for $i \neq 0, i_{2}$. Moreover, $\xi$ is an isomorphism of $\mathfrak{g}_{I \backslash\left\{0, i_{2}\right\} \text {-geometric crystals. }}$. 
Now the $\mathfrak{g}$-geometric crystal $\mathcal{V}(\mathfrak{g})$ is obtained by patching $X_{1}$ and $X_{2}$ by $\xi$. The relations (1)-(4) in Definition 2.1 are obvious for $(i, j) \neq\left(0, i_{2}\right),\left(i_{2}, 0\right)$ and we check these relations for $(i, j)=\left(0, i_{2}\right),\left(i_{2}, 0\right)$ by hand. In this paper we choose $\eta=\sigma \varpi_{1}$ for some Dynkin diagram automorphism $\sigma$ except the case $\mathfrak{g}=A_{2 n}^{(2)}$.

We then show that the ultra-discretization of $\mathcal{V}(\mathfrak{g})$ is isomorphic to the crystal $B_{\infty}\left(\varpi_{1}\right)$ of the Langlands dual $\mathfrak{g}^{L}$.

The organization of the paper is as follows. In $₫ 2$ we review basic facts about geometric crystals following [1], 14, [15]. In $\oint 3$, we shall recall the notion of the limit of perfect crystals and give examples of the limit $B_{\infty}\left(\mathfrak{g}^{L}\right)$ of perfect crystals following [6. In $\$$, we present the explicit form of the fundamental representation $W\left(\varpi_{1}\right)$ for each affine Lie algebra $\mathfrak{g}$. In $₫ 5$, we shall construct the affine geometric crystal $\mathcal{V}(\mathfrak{g})$ associated with the fundamental representation $W\left(\varpi_{1}\right)$. In 86 , we prove that the ultra-discretization $\mathcal{U} D(\mathcal{V}(\mathfrak{g}))$ is isomorphic to $B_{\infty}\left(\mathfrak{g}^{L}\right)$.

\section{Geometric CRYSTALS}

In this section, we review Kac-Moody groups and geometric crystals following [1], 4], 5], 11, 14, [16.

2.1. Kac-Moody algebras and Kac-Moody groups. Fix a symmetrizable generalized Cartan matrix $A=\left(a_{i j}\right)_{i, j \in I}$ with a finite index set $I$. Let $\left(\mathfrak{t},\left\{\alpha_{i}\right\}_{i \in I}\right.$, $\left.\left\{\alpha_{i}^{\vee}\right\}_{i \in I}\right)$ be the associated root data, where $\mathfrak{t}$ is a vector space over $\mathbb{C}$ and $\left\{\alpha_{i}\right\}_{i \in I} \subset$ $\mathfrak{t}^{*}$ and $\left\{\alpha_{i}^{\vee}\right\}_{i \in I} \subset \mathfrak{t}$ are linearly independent satisfying $\alpha_{j}\left(\alpha_{i}^{\vee}\right)=a_{i j}$.

The Kac-Moody Lie algebra $\mathfrak{g}=\mathfrak{g}(A)$ associated with $A$ is the Lie algebra over $\mathbb{C}$ generated by $\mathfrak{t}$ and the Chevalley generators $e_{i}$ and $f_{i}(i \in I)$ with the usual defining relations ([5, [16]). There exists a root space decomposition $\mathfrak{g}=\bigoplus_{\alpha \in \mathfrak{t}^{*}} \mathfrak{g}_{\alpha}$. Denote the set of roots by $\Delta:=\left\{\alpha \in \mathfrak{t}^{*} \mid \alpha \neq 0, \mathfrak{g}_{\alpha} \neq(0)\right\}$. Set $Q=\sum_{i} \mathbb{Z} \alpha_{i}, Q_{+}=$ $\sum_{i} \mathbb{Z}_{\geq 0} \alpha_{i}, Q^{\vee}=\sum_{i} \mathbb{Z} \alpha_{i}^{\vee}$ and $\Delta_{+}=\Delta \cap Q_{+}$. An element of $\Delta_{+}$is called a positive root. Let $P \subset \mathfrak{t}^{*}$ be a weight lattice such that $\mathbb{C} \otimes P=\mathfrak{t}^{*}$, whose element is called a weight.

Define the simple reflections $s_{i} \in \operatorname{Aut}(\mathfrak{t})(i \in I)$ by $s_{i}(h):=h-\alpha_{i}(h) \alpha_{i}^{\vee}$, which generate the Weyl group $W$. They induce the action of $W$ on $\mathfrak{t}^{*}$ by $s_{i}(\lambda):=$ $\lambda-\lambda\left(\alpha_{i}^{\vee}\right) \alpha_{i}$. Set $\Delta^{\mathrm{re}}:=\left\{w\left(\alpha_{i}\right) \mid w \in W, i \in I\right\}$, whose elements are called real roots.

Let $G$ be the Kac-Moody group associated with $\mathfrak{g}\left([16)\right.$. Let $U_{\alpha}:=\exp \mathfrak{g}_{\alpha}$ $\left(\alpha \in \Delta^{\mathrm{re}}\right.$ ) be the one-parameter subgroup of $G$. The group $G$ is generated by $U_{\alpha}$ $\left(\alpha \in \Delta^{\mathrm{re}}\right)$. Let $U^{ \pm}$be the subgroup generated by $U_{ \pm \alpha}\left(\alpha \in \Delta_{+}^{\mathrm{re}}=\Delta^{\mathrm{re}} \cap Q_{+}\right)$, i.e., $U^{ \pm}:=\left\langle U_{ \pm \alpha} \mid \alpha \in \Delta_{+}^{\mathrm{re}}\right\rangle$.

For any $i \in I$, there exists a unique group homomorphism $\phi_{i}: S L_{2}(\mathbb{C}) \rightarrow G$ such that

$$
\phi_{i}\left(\left(\begin{array}{ll}
1 & t \\
0 & 1
\end{array}\right)\right)=\exp \left(t e_{i}\right), \phi_{i}\left(\left(\begin{array}{ll}
1 & 0 \\
t & 1
\end{array}\right)\right)=\exp \left(t f_{i}\right) \quad(t \in \mathbb{C}) .
$$

Set $\alpha_{i}^{\vee}(c):=\phi_{i}\left(\left(\begin{array}{cc}c & 0 \\ 0 & c^{-1}\end{array}\right)\right), x_{i}(t):=\exp \left(t e_{i}\right), y_{i}(t):=\exp \left(t f_{i}\right), G_{i}:=\phi_{i}\left(S L_{2}(\mathbb{C})\right)$, $T_{i}:=\alpha_{i}^{\vee}\left(\mathbb{C}^{\times}\right)$and $N_{i}:=N_{G_{i}}\left(T_{i}\right)$. Let $T$ be the subgroup of $G$ with the Lie algebra $\mathfrak{t}$, which is called a maximal torus in $G$, and let $B^{ \pm}=U^{ \pm} T$ be the Borel subgroup of $G$. Let $N$ be the subgroup of $G$ generated by the $N_{i}$ 's. Then we have the isomorphism $\phi: W \stackrel{\sim}{\longrightarrow} N / T$ defined by $\phi\left(s_{i}\right)=N_{i} T / T$. An element $\bar{s}_{i}:=x_{i}(-1) y_{i}(1) x_{i}(-1)=$ $\phi_{i}\left(\left(\begin{array}{cc}0 & -1 \\ 1 & 0\end{array}\right)\right)$ in $N_{G}(T)$ is a representative of $s_{i} \in W=N_{G}(T) / T$. 
2.2. Geometric crystals. Let $W$ be the Weyl group associated with $\mathfrak{g}$. Define $R(w)$ for $w \in W$ by

$$
R(w):=\left\{\left(i_{1}, i_{2}, \cdots, i_{l}\right) \in I^{l} \mid w=s_{i_{1}} s_{i_{2}} \cdots s_{i_{l}}\right\},
$$

where $l$ is the length of $w$, i.e., $R(w)$ is the set of reduced expressions of $w$.

Let $X$ be a variety, $\gamma_{i}: X \rightarrow \mathbb{C}$ and $\varepsilon_{i}: X \longrightarrow \mathbb{C}(i \in I)$ rational functions on $X$, and $e_{i}: \mathbb{C}^{\times} \times X \longrightarrow X\left((c, x) \mapsto e_{i}^{c}(x)\right)$ a rational $\mathbb{C}^{\times}$-action. For $w \in W$ and $\mathbf{i}=\left(i_{1}, \cdots, i_{l}\right) \in R(w)$, set $\alpha^{(j)}:=s_{i_{l}} \cdots s_{i_{j+1}}\left(\alpha_{i_{j}}\right)(1 \leq j \leq l)$ and

$$
\begin{aligned}
e_{\mathbf{i}}: T \times X & \rightarrow X \\
(t, x) & \mapsto e_{\mathbf{i}}^{t}(x):=e_{i_{1}}^{\alpha^{(1)}(t)} e_{i_{2}}^{\alpha^{(2)}(t)} \cdots e_{i_{l}}^{\alpha^{(l)}(t)}(x) .
\end{aligned}
$$

Definition 2.1. A quadruple $\left(X,\left\{e_{i}\right\}_{i \in I},\left\{\gamma_{i}\right\}_{i \in I},\left\{\varepsilon_{i}\right\}_{i \in I}\right)$ is a $G$ (or $\mathfrak{g}$ )-geometric crystal if

(1) $\{1\} \times X \subset \operatorname{dom}\left(e_{i}\right)$ for any $i \in I$.

(2) $\gamma_{j}\left(e_{i}^{c}(x)\right)=c^{a_{i j}} \gamma_{j}(x)$.

(3) $e_{\mathbf{i}}=e_{\mathbf{i}^{\prime}}$ for any $w \in W$ and $\mathbf{i}$. $\mathbf{i}^{\prime} \in R(w)$.

(4) $\varepsilon_{i}\left(e_{i}^{c}(x)\right)=c^{-1} \varepsilon_{i}(x)$.

Note that the condition (3) is equivalent to the following so-called Verma relations:

$$
\begin{array}{ll}
e_{i}^{c_{1}} e_{j}^{c_{2}}=e_{j}^{c_{2}} e_{i}^{c_{1}} & \text { if } a_{i j}=a_{j i}=0, \\
e_{i}^{c_{1}} e_{j}^{c_{1} c_{2}} e_{i}^{c_{2}}=e_{j}^{c_{2}} e_{i}^{c_{1} c_{2}} e_{j}^{c_{1}} & \text { if } a_{i j}=a_{j i}=-1, \\
e_{i}^{c_{1}} e_{j}^{c_{1}^{2} c_{2}} e_{i}^{c_{1} c_{2}} e_{j}^{c_{2}}=e_{j}^{c_{2}} e_{i}^{c_{1} c_{2}} e_{j}^{c_{1}^{2} c_{2}} e_{i}^{c_{1}} & \text { if } a_{i j}=-2, a_{j i}=-1, \\
e_{i}^{c_{1}} e_{j}^{c_{1}^{3} c_{2}} e_{i}^{c_{1}^{2} c_{2}} e_{j}^{c_{1}^{3} c_{2}^{2}} e_{i}^{c_{1} c_{2}} e_{j}^{c_{2}}=e_{j}^{c_{2}} e_{i}^{c_{1} c_{2}} e_{j}^{c_{1}^{3} c_{2}^{2}} e_{i}^{c_{1}^{2} c_{2}} e_{j}^{c_{1}^{3} c_{2}} e_{i}^{c_{1}} & \text { if } a_{i j}=-3, a_{j i}=-1
\end{array}
$$

Note that the last formula is different from the one in [1, 14, 15, which seems to be incorrect.

2.3. Geometric crystal on Schubert cell. Let $X:=G / B$ be the flag variety, which is the inductive limit of finite-dimensional projective varieties. For $w \in W$, let $X_{w}:=B w B / B \subset X$ be the Schubert cell associated with $w$, which has a natural geometric crystal structure ([1, [14]). For $\mathbf{i}=\left(i_{1}, \cdots, i_{k}\right) \in R(w)$, set

$$
B_{\mathbf{i}}^{-}:=\left\{Y_{\mathbf{i}}\left(c_{1}, \cdots, c_{k}\right):=Y_{i_{1}}\left(c_{1}\right) \cdots Y_{i_{l}}\left(c_{k}\right) \mid c_{1}, \cdots, c_{k} \in \mathbb{C}^{\times}\right\} \subset B^{-}
$$

where $Y_{i}(c):=y_{i}\left(c^{-1}\right) \alpha_{i}^{\vee}(c)$. Then $B_{\mathbf{i}}^{-}$is birationally isomorphic to $X_{w}$ and endowed with the induced geometric crystal structure. The explicit form of the action $e_{i}^{c}$ on $B_{\mathbf{i}}^{-}$is given by

$$
e_{i}^{c}\left(Y_{i_{1}}\left(c_{1}\right) \cdots Y_{i_{l}}\left(c_{k}\right)\right)=Y_{i_{1}}\left(\mathcal{C}_{1}\right) \cdots Y_{i_{l}}\left(\mathcal{C}_{k}\right)
$$

where

$$
\mathcal{C}_{j}:=c_{j} \cdot \frac{\sum_{1 \leq m \leq j, i_{m}=i} \frac{c}{c_{1}^{a_{i_{1}, i}} \cdots c_{m-1}^{a_{i_{m}-1, i}} c_{m}}+\sum_{j<m \leq k, i_{m}=i} \frac{1}{c_{1 \leq m<j, i_{m}=i}^{a_{i_{1}, i}} \cdots c_{m-1}^{a_{i_{m}-1, i}} c_{m}}}{\frac{c}{c_{1}^{a_{i_{1}, i}} \cdots c_{m-1}^{a_{i_{m}, i}} c_{m}}+\sum_{j \leq m \leq k, i_{m}=i} \frac{1}{c_{1}^{a_{i_{1}, i}} \cdots c_{m-1}^{a_{i_{m}-1, i}} c_{m}}}
$$


We also have the explicit forms of the rational functions $\varepsilon_{i}$ and $\gamma_{i}$ :

$$
\begin{gathered}
\varepsilon_{i}\left(Y_{i_{1}}\left(c_{1}\right) \cdots Y_{i_{l}}\left(c_{k}\right)\right)=\sum_{1 \leq m \leq k, i_{m}=i} \frac{1}{c_{1}^{a_{i_{1}, i}} \cdots c_{m-1}^{a_{i_{m}-1} i} c_{m}}, \\
\gamma_{i}\left(Y_{i_{1}}\left(c_{1}\right) \cdots Y_{i_{l}}\left(c_{k}\right)\right)=c_{1}^{a_{i_{1}, i}} \cdots c_{k}^{a_{i_{k}, i}} .
\end{gathered}
$$

2.4. Positive structure, ultra-discretization and tropicalization. Let us recall the notions of positive structure and ultra-discretization/tropicalization.

The setting below is the same as in 15 . Set $R:=\mathbb{C}(c)$ and define

$$
\begin{array}{cccc}
v: \quad R \backslash\{0\} & \longrightarrow & \mathbb{Z} \\
& f(c) & \mapsto & \operatorname{deg}(f(c)) .
\end{array}
$$

Here deg is the degree of the pole at $c=\infty$. Note that for $f_{1}, f_{2} \in R \backslash\{0\}$, we have

$$
v\left(f_{1} f_{2}\right)=v\left(f_{1}\right)+v\left(f_{2}\right), \quad v\left(\frac{f_{1}}{f_{2}}\right)=v\left(f_{1}\right)-v\left(f_{2}\right) .
$$

We say that a non-zero rational function $f(c) \in \mathbb{C}(c)$ is positive if $f$ can be expressed as a ratio of polynomials with positive coefficients. If $f_{1}, f_{2} \in R$ are positive, then we have

$$
v\left(f_{1}+f_{2}\right)=\max \left(v\left(f_{1}\right), v\left(f_{2}\right)\right) .
$$

Let $T \simeq\left(\mathbb{C}^{\times}\right)^{l}$ be an algebraic torus over $\mathbb{C}$ and $X^{*}(T):=\operatorname{Hom}\left(T, \mathbb{C}^{\times}\right)$(resp. $\left.X_{*}(T):=\operatorname{Hom}\left(\mathbb{C}^{\times}, T\right)\right)$ be the lattice of characters (resp. co-characters) of $T$. A non-zero rational function on an algebraic torus $T$ is called positive if it is written as $g / h$ where $g$ and $h$ are positive linear combinations of characters of $T$.

Definition 2.2. Let $f: T \rightarrow T^{\prime}$ be a rational mapping between two algebraic tori $T$ and $T^{\prime}$. We say that $f$ is positive, if $\chi \circ f$ is positive for any character $\chi: T^{\prime} \rightarrow \mathbb{C}$.

Denote by $\operatorname{Mor}^{+}\left(T, T^{\prime}\right)$ the set of positive rational mappings from $T$ to $T^{\prime}$.

Lemma 2.3 ([1]). For any $f \in \operatorname{Mor}^{+}\left(T_{1}, T_{2}\right)$ and $g \in \operatorname{Mor}^{+}\left(T_{2}, T_{3}\right)$, the composition $g \circ f$ is well-defined and belongs to $\operatorname{Mor}^{+}\left(T_{1}, T_{3}\right)$.

By Lemma 2.3. we can define a category $\mathcal{T}_{+}$whose objects are algebraic tori over $\mathbb{C}$ and whose arrows are positive rational mappings.

Let $f: T \rightarrow T^{\prime}$ be a positive rational mapping of algebraic tori $T$ and $T^{\prime}$. We define a map $\widehat{f}: X_{*}(T) \rightarrow X_{*}\left(T^{\prime}\right)$ by

$$
\langle\chi, \widehat{f}(\xi)\rangle=v(\chi \circ f \circ \xi),
$$

where $\chi \in X^{*}\left(T^{\prime}\right)$ and $\xi \in X_{*}(T)$.

Lemma 2.4 ([1). For any algebraic tori $T_{1}, T_{2}, T_{3}$, and positive rational mappings $f \in \operatorname{Mor}^{+}\left(T_{1}, T_{2}\right), g \in \operatorname{Mor}^{+}\left(T_{2}, T_{3}\right)$, we have $\widehat{g \circ f}=\widehat{g} \circ \widehat{f}$.

By this lemma, we obtain a functor

$$
\begin{array}{cccc}
\mathcal{U} D: & \mathcal{T}_{+} & & \text {Set } \\
T & & & X_{*}(T) \\
\left(f: T \rightarrow T^{\prime}\right) & \mapsto & \left(\widehat{f}: X_{*}(T) \rightarrow X_{*}\left(T^{\prime}\right)\right) .
\end{array}
$$


Let us come back to the situation in $\$ 2.2$. Hence $G$ is a Kac-Moody group and $T$ is its Cartan subgroup.

Definition 2.5 ([1]). Let $\chi=\left(X,\left\{e_{i}\right\}_{i \in I},\left\{\gamma_{i}\right\}_{i \in I},\left\{\varepsilon_{i}\right\}_{i \in I}\right)$ be a $G$-geometric crystal, $T^{\prime}$ an algebraic torus and $\theta: T^{\prime} \rightarrow X$ a birational mapping. The mapping $\theta$ is called a positive structure on $\chi$ if it satisfies

(1) for any $i \in I$ the rational functions $\gamma_{i} \circ \theta: T^{\prime} \rightarrow \mathbb{C}$ and $\varepsilon_{i} \circ \theta: T^{\prime} \rightarrow \mathbb{C}$ are positive,

(2) for any $i \in I$, the rational mapping $e_{i, \theta}: \mathbb{C}^{\times} \times T^{\prime} \rightarrow T^{\prime}$ defined by $e_{i, \theta}(c, t):=$ $\theta^{-1} \circ e_{i}^{c} \circ \theta(t)$ is positive.

Let $\theta: T^{\prime} \rightarrow X$ be a positive structure on a geometric crystal $\chi=\left(X,\left\{e_{i}\right\}_{i \in I}\right.$, $\left.\left\{\gamma_{i}\right\}_{i \in I},\left\{\varepsilon_{i}\right\}_{i \in I}\right)$. Applying the functor $\mathcal{U} D$ to the positive rational mappings $e_{i, \theta}: \mathbb{C}^{\times} \times T^{\prime} \rightarrow T^{\prime}$ and $\gamma_{i} \circ \theta, \varepsilon_{i} \circ \theta: T^{\prime} \rightarrow \mathbb{C}^{\times}$, we obtain

$$
\begin{array}{rcrl}
\tilde{e}_{i}:=\mathcal{U} D\left(e_{i, \theta}\right): & \mathbb{Z} \times X_{*}\left(T^{\prime}\right) \rightarrow X_{*}\left(T^{\prime}\right), \\
\mathrm{wt}_{i}:=\mathcal{U} D\left(\gamma_{i} \circ \theta\right), \varepsilon_{i}:=\mathcal{U} D\left(\varepsilon_{i} \circ \theta\right): & X_{*}\left(T^{\prime}\right) \rightarrow \mathbb{Z} .
\end{array}
$$

Hence the quadruple $\left(X_{*}\left(T^{\prime}\right),\left\{\tilde{e}_{i}\right\}_{i \in I},\left\{\mathrm{wt}_{i}\right\}_{i \in I},\left\{\varepsilon_{i}\right\}_{i \in I}\right)$ is a free pre-crystal structure (see [1, 2.2]) and we denote it by $\mathcal{U} D_{\theta, T^{\prime}}(\chi)$. We thus have the following theorem:

Theorem 2.6 ([1, 14]). For any geometric crystal $\chi=\left(X,\left\{e_{i}\right\}_{i \in I},\left\{\gamma_{i}\right\}_{i \in I},\left\{\varepsilon_{i}\right\}_{i \in I}\right)$ and a positive structure $\theta: T^{\prime} \rightarrow X$, the associated pre-crystalU $D_{\theta, T^{\prime}}(\chi)$ is a crystal (see [1, 2.2]).

Now, let $\mathcal{G} C^{+}$be the category whose object is a triplet $\left(\chi, T^{\prime}, \theta\right)$, where $\chi=$ $\left(X,\left\{e_{i}\right\},\left\{\gamma_{i}\right\},\left\{\varepsilon_{i}\right\}\right)$ is a geometric crystal and $\theta: T^{\prime} \rightarrow X$ is a positive structure on $\chi$, and the morphism $f:\left(\chi_{1}, T_{1}^{\prime}, \theta_{1}\right) \rightarrow\left(\chi_{2}, T_{2}^{\prime}, \theta_{2}\right)$ is given by a morphism $\varphi: X_{1} \longrightarrow X_{2}$ of geometric crystals such that

$$
f:=\theta_{2}^{-1} \circ \varphi \circ \theta_{1}: T_{1}^{\prime} \longrightarrow T_{2}^{\prime}
$$

is a positive rational mapping. Let $\mathcal{C} R$ be the category of crystals. Then by the theorem above, we have

Corollary 2.7. $\mathcal{U D}$ defines a functor

$$
\begin{array}{ccc}
\mathcal{U} D: \mathcal{G} C^{+} & \longrightarrow & \mathcal{C} R, \\
\left(\chi, T^{\prime}, \theta\right) & \mapsto & X_{*}\left(T^{\prime}\right), \\
\left(f:\left(\chi_{1}, T_{1}^{\prime}, \theta_{1}\right) \rightarrow\left(\chi_{2}, T_{2}^{\prime}, \theta_{2}\right)\right) & \mapsto & \left(\widehat{f}: X_{*}\left(T_{1}^{\prime}\right) \rightarrow X_{*}\left(T_{2}^{\prime}\right)\right) .
\end{array}
$$

We call the functor $\mathcal{U} D$ "ultra-discretization" as in [14, [15], while it is called "tropicalization" in [1. For a crystal $B$, if there exists a geometric crystal $\chi$ and a positive structure $\theta: T^{\prime} \rightarrow X$ on $\chi$ such that $\mathcal{U} D\left(\chi, T^{\prime}, \theta\right) \simeq B$ as crystals, we call an object $\left(\chi, T^{\prime}, \theta\right)$ in $\mathcal{G} C^{+}$a tropicalization of $B$. 


\section{LIMITS OF PERFECT CRYSTALS}

We review the limits of perfect crystals following [6]. (See also [7, [8.)

3.1. Crystals. First we recall the notion of crystals, which is obtained by abstracting the combinatorial properties of crystal bases.

Definition 3.1. A crystal $B$ is a set endowed with the following maps:

$$
\begin{aligned}
& \text { wt: } B \longrightarrow P, \\
& \varepsilon_{i}: B \longrightarrow \mathbb{Z} \sqcup\{-\infty\}, \quad \varphi_{i}: B \longrightarrow \mathbb{Z} \sqcup\{-\infty\} \quad \text { for } i \in I, \\
& \tilde{e}_{i}: B \sqcup\{0\} \longrightarrow B \sqcup\{0\}, \quad \tilde{f}_{i}: B \sqcup\{0\} \longrightarrow B \sqcup\{0\} \quad \text { for } \quad i \in I, \\
& \tilde{e}_{i}(0)=\tilde{f}_{i}(0)=0 .
\end{aligned}
$$

Those maps satisfy the following axioms: for all $b, b_{1}, b_{2} \in B$, we have

$$
\begin{aligned}
& \varphi_{i}(b)=\varepsilon_{i}(b)+\left\langle\alpha_{i}^{\vee}, \operatorname{wt}(b)\right\rangle, \\
& \operatorname{wt}\left(\tilde{e}_{i} b\right)=\operatorname{wt}(b)+\alpha_{i} \text { if } \tilde{e}_{i} b \in B, \\
& \operatorname{wt}\left(\tilde{f}_{i} b\right)=\operatorname{wt}(b)-\alpha_{i} \text { if } \tilde{f}_{i} b \in B, \\
& \tilde{e}_{i} b_{2}=b_{1} \Longleftrightarrow \tilde{f}_{i} b_{1}=b_{2}, \\
& \varepsilon_{i}(b)=-\infty \Longrightarrow \tilde{e}_{i} b=\tilde{f}_{i} b=0 .
\end{aligned}
$$

The following tensor product structure is one of the most crucial properties of crystals.

Theorem 3.2. Let $B_{1}$ and $B_{2}$ be crystals. Set $B_{1} \otimes B_{2}:=\left\{b_{1} \otimes b_{2} \mid b_{j} \in B_{j}(j=1,2)\right\}$. Then we have

(1) $B_{1} \otimes B_{2}$ is a crystal,

(2) for $b_{1} \in B_{1}$ and $b_{2} \in B_{2}$, we have

$$
\begin{aligned}
& \tilde{f}_{i}\left(b_{1} \otimes b_{2}\right)= \begin{cases}\tilde{f}_{i} b_{1} \otimes b_{2} & \text { if } \varphi_{i}\left(b_{1}\right)>\varepsilon_{i}\left(b_{2}\right), \\
b_{1} \otimes \tilde{f}_{i} b_{2} & \text { if } \varphi_{i}\left(b_{1}\right) \leq \varepsilon_{i}\left(b_{2}\right) ;\end{cases} \\
& \tilde{e}_{i}\left(b_{1} \otimes b_{2}\right)= \begin{cases}b_{1} \otimes \tilde{e}_{i} b_{2} & \text { if } \varphi_{i}\left(b_{1}\right)<\varepsilon_{i}\left(b_{2}\right), \\
\tilde{e}_{i} b_{1} \otimes b_{2} & \text { if } \varphi_{i}\left(b_{1}\right) \geq \varepsilon_{i}\left(b_{2}\right) .\end{cases}
\end{aligned}
$$

Definition 3.3. Let $B_{1}$ and $B_{2}$ be crystals. A morphism of crystals $\psi: B_{1} \longrightarrow B_{2}$ is a map $\psi: B_{1} \sqcup\{0\} \longrightarrow B_{2} \sqcup\{0\}$ satisfying

(1) $\psi(0)=0, \psi\left(B_{1}\right) \subset B_{2}$,

(2) for $b, b^{\prime} \in B_{1}, \tilde{f}_{i} b=b^{\prime}$ implies $\tilde{f}_{i} \psi(b)=\psi\left(b^{\prime}\right)$,

(3) $\operatorname{wt}(\psi(b))=\operatorname{wt}(b), \quad \varepsilon_{i}(\psi(b))=\varepsilon_{i}(b), \quad \varphi_{i}(\psi(b))=\varphi_{i}(b) \quad$ for any $b \in B_{1}$.

In particular, a bijective morphism is called an isomorphism of crystals.

Example 3.4. (1) If $(L, B)$ is a crystal base, then $B$ is a crystal.

(2) For the crystal base $(L(\infty), B(\infty))$ of the subalgebra $U_{q}^{-}(\mathfrak{g})$ of the quantum algebra $U_{q}(\mathfrak{g}), B(\infty)$ is a crystal.

(3) For $\lambda \in P$, set $T_{\lambda}:=\left\{t_{\lambda}\right\}$. We define a crystal structure on $T_{\lambda}$ by

$$
\tilde{e}_{i}\left(t_{\lambda}\right)=\tilde{f}_{i}\left(t_{\lambda}\right)=0, \quad \varepsilon_{i}\left(t_{\lambda}\right)=\varphi_{i}\left(t_{\lambda}\right)=-\infty, \quad \operatorname{wt}\left(t_{\lambda}\right)=\lambda .
$$


Definition 3.5. To a crystal $B$, a colored oriented graph is associated by

$$
b_{1} \stackrel{i}{\longrightarrow} b_{2} \Longleftrightarrow \tilde{f}_{i} b_{1}=b_{2} \text {. }
$$

We call this graph the crystal graph of $B$.

3.2. Affine weights. Let $\mathfrak{g}$ be an affine Lie algebra, and let the sets $\mathfrak{t},\left\{\alpha_{i}\right\}_{i \in I}$ and $\left\{\alpha_{i}^{\vee}\right\}_{i \in I}$ be as in $\$ 2.1$. We take $\mathfrak{t}$ so that $\operatorname{dim} \mathfrak{t}=\sharp I+1$. Let $\delta \in Q_{+}$be a unique element satisfying

$$
\left\{\lambda \in Q \mid\left\langle\alpha_{i}^{\vee}, \lambda\right\rangle=0 \text { for any } i \in I\right\}=\mathbb{Z} \delta,
$$

and let $\mathbf{c} \in \sum_{i} \mathbb{Z}_{\geq 0} \alpha_{i}^{\vee} \subset \mathfrak{g}$ be a unique central element satisfying

$$
\left\{h \in Q^{\vee} \mid\left\langle h, \alpha_{i}\right\rangle=0 \text { for any } i \in I\right\}=\mathbb{Z} \mathbf{c} .
$$

We write $([4,6.1])$

$$
\mathbf{c}=\sum_{i} a_{i}^{\vee} \alpha_{i}^{\vee}, \quad \delta=\sum_{i} a_{i} \alpha_{i}
$$

Let $\left(\right.$, ) be the non-degenerate $W$-invariant symmetric bilinear form on $\mathfrak{t}^{*}$ normalized by $(\delta, \lambda)=\langle\mathbf{c}, \lambda\rangle$ for $\lambda \in \mathfrak{t}^{*}$. Let us set $\mathfrak{t}_{\mathrm{cl}}^{*}:=\mathfrak{t}^{*} / \mathbb{C} \delta$ and let $\mathrm{cl}: \mathfrak{t}^{*} \longrightarrow \mathfrak{t}_{\mathrm{cl}}^{*}$ be the canonical projection. Then we have $\mathfrak{t}_{\mathrm{cl}}^{*} \cong \bigoplus_{i}\left(\mathbb{C} \alpha_{i}^{\vee}\right)^{*}$. Set $\mathfrak{t}_{0}^{*}:=\left\{\lambda \in \mathfrak{t}^{*} \mid\langle\mathbf{c}, \lambda\rangle=0\right\}$, $\left(\mathfrak{t}_{\mathrm{cl}}^{*}\right)_{0}:=\operatorname{cl}\left(\mathfrak{t}_{0}^{*}\right)$. Then we have a positive-definite symmetric form on $\left(\mathfrak{t}_{\mathrm{cl}}^{*}\right)_{0}$ induced by the one on $\mathfrak{t}^{*}$. Let $\Lambda_{i} \in \mathfrak{t}_{\mathrm{cl}}^{*}(i \in I)$ be a weight such that $\left\langle\alpha_{i}^{\vee}, \Lambda_{j}\right\rangle=\delta_{i, j}$, which is called a fundamental weight. We choose $P$ so that $P_{\mathrm{cl}}:=\mathrm{cl}(P)$ coincides with $\bigoplus_{i \in I} \mathbb{Z} \Lambda_{i}$ and we call $P_{\mathrm{cl}}$ the classical weight lattice.

3.3. Definitions of a perfect crystal and its limit. Let $\mathfrak{g}$ be an affine Lie algebra, $P_{\mathrm{cl}}$ the classical weight lattice as above and set $\left(P_{\mathrm{cl}}\right)_{l}^{+}:=\left\{\lambda \in P_{\mathrm{cl}}\langle\mathbf{c}, \lambda\rangle=l\right.$, $\left.\left\langle\alpha_{i}^{\vee}, \lambda\right\rangle \geq 0\right\}$ for $l \in \mathbb{Z}_{>0}$.

Definition 3.6. We say that a crystal $B$ is perfect of level $l$ if

(1) $B \otimes B$ is connected as a crystal graph.

(2) There exists $\lambda_{0} \in P_{\mathrm{cl}}$ such that

$$
\operatorname{wt}(B) \subset \lambda_{0}+\sum_{i \neq 0} \mathbb{Z}_{\leq 0} \operatorname{cl}\left(\alpha_{i}\right), \quad \sharp B_{\lambda_{0}}=1 .
$$

(3) There exists a finite-dimensional $U_{q}^{\prime}(\mathfrak{g})$-module $V$ with a crystal pseudobase $B_{p s}$ such that $B \cong B_{p s} / \pm 1$.

(4) The maps $\varepsilon, \varphi: B^{\text {min }}:=\{b \in B \mid\langle c, \varepsilon(b)\rangle=l\} \longrightarrow\left(P_{\mathrm{cl}}^{+}\right)_{l}$ are bijective, where $\varepsilon(b):=\sum_{i} \varepsilon_{i}(b) \Lambda_{i}$ and $\varphi(b):=\sum_{i} \varphi_{i}(b) \Lambda_{i}$.

Let $\left\{B_{l}\right\}_{l \geq 1}$ be a family of perfect crystals of level $l$ and set $J:=\{(l, b) \mid l>0$, $\left.b \in B_{l}^{\min }\right\}$.

Definition 3.7. A crystal $B_{\infty}$ with an element $b_{\infty}$ is called the limit of $\left\{B_{l}\right\}_{l \geq 1}$ if

(1) $\operatorname{wt}\left(b_{\infty}\right)=\varepsilon\left(b_{\infty}\right)=\varphi\left(b_{\infty}\right)=0$.

(2) For any $(l, b) \in J$, there exists an embedding of crystals:

$$
\begin{aligned}
f_{(l, b)}: \quad T_{\varepsilon(b)} \otimes B_{l} \otimes T_{-\varphi(b)} & \hookrightarrow B_{\infty} \\
t_{\varepsilon(b)} \otimes b \otimes t_{-\varphi(b)} & \mapsto b_{\infty} .
\end{aligned}
$$

(3) $B_{\infty}=\bigcup_{(l, b) \in J} \operatorname{Im} f_{(l, b)}$. 
As for the crystal $T_{\lambda}$, see Example 3.4 (3). If the limit of a family $\left\{B_{l}\right\}$ exists, we say that $\left\{B_{l}\right\}$ is a coherent family of perfect crystals.

The following is one of the most important properties of the limits of perfect crystals.

Proposition $3.8(\underline{6})$. Let $B(\infty)$ be the crystal as in Example 3.4 (2). Then we have the following isomorphism of crystals:

$$
B(\infty) \otimes B_{\infty} \stackrel{\sim}{\longrightarrow} B(\infty) .
$$

In the rest of this section, for each affine Lie algebra $\mathfrak{g}=A_{n}^{(1)}, B_{n}^{(1)}, C_{n}^{(1)}, D_{n}^{(1)}$, $A_{2 n-1}^{(2)}, D_{n+1}^{(2)}, A_{2 n}^{(2)}$, we explicitly describe an example of $B_{\infty}=B_{\infty}(\mathfrak{g})$ following [6].

3.4. $A_{n}^{(1)}(n \geq 2)$. The Cartan matrix $\left(a_{i j}\right)_{i, j \in I}(I:=\{0,1, \cdots, n\})$ of type $A_{n}^{(1)}$ is

$$
a_{i j}= \begin{cases}2 & \text { if } i=j, \\ -1 & \text { if } i \equiv j \pm 1 \quad \bmod n+1, \\ 0 & \text { otherwise. }\end{cases}
$$

We have $\mathbf{c}=\sum_{i \in I} \alpha_{i}^{\vee}$ and $\delta=\sum_{i \in I} \alpha_{i}$. A limit of perfect crystals is given as follows:

$$
B_{\infty}\left(A_{n}^{(1)}\right)=\left\{\left(b_{1}, \cdots, b_{n+1}\right) \in \mathbb{Z}^{n+1} \mid \sum_{i=1}^{n+1} b_{i}=0\right\},
$$

for $b=\left(b_{1}, \cdots, b_{n+1}\right) \in B_{\infty}\left(A_{n}^{(1)}\right)$. We have

$$
\left\{\begin{array}{l}
\tilde{e}_{0} b=\left(b_{1}-1, b_{2}, \cdots, b_{n}, b_{n+1}+1\right), \\
\tilde{e}_{i} b=\left(b_{1}, \cdots, b_{i}+1, b_{i+1}-1, \cdots, b_{n+1}\right)(i=1, \cdots, n), \\
\tilde{f}_{i}=\tilde{e}_{i}^{-1}
\end{array}\right.
$$

and

$$
\left\{\begin{array}{l}
\operatorname{wt}(b)=\left(b_{n+1}-b_{1}\right) \Lambda_{0}+\sum_{i=1}^{n}\left(b_{i}-b_{i+1}\right) \Lambda_{i}, \\
\varepsilon_{0}(b)=b_{1}, \quad \varepsilon_{i}(b)=b_{i+1}(i=1, \cdots, n), \\
\varphi_{0}(b)=b_{n+1}, \quad \varphi_{i}(b)=b_{i}(i=1, \cdots, n) .
\end{array}\right.
$$

3.5. $B_{n}^{(1)}(n \geq 3)$. The Cartan matrix $\left(a_{i j}\right)_{i, j \in I}(I:=\{0,1, \cdots, n\})$ of type $B_{n}^{(1)}$ is

$$
a_{i j}= \begin{cases}2 & i=j, \\ -1 & |i-j|=1 \text { and }(i, j) \neq(0,1),(1,0),(n, n-1) \text { or }(i, j)=(0,2),(2,0), \\ -2 & (i, j)=(n, n-1), \\ 0 & \text { otherwise. }\end{cases}
$$

The Dynkin diagram is

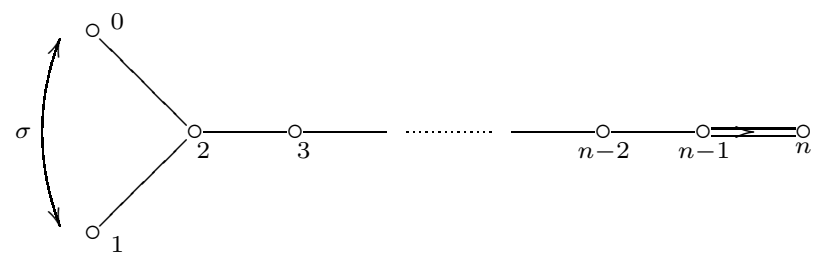


where $\sigma$ is a Dynkin diagram automorphism which we use later. We have

$$
\mathbf{c}=\alpha_{0}^{\vee}+\alpha_{1}^{\vee}+2 \sum_{i=2}^{n-1} \alpha_{i}^{\vee}+\alpha_{n}^{\vee}, \quad \delta=\alpha_{0}+\alpha_{1}+2 \sum_{i=2}^{n} \alpha_{i} .
$$

A limit of perfect crystals is given as follows:

$$
\begin{aligned}
B_{\infty}\left(B_{n}^{(1)}\right)=\left\{\left(b_{1}, \cdots, b_{n}, \bar{b}_{n}, \cdots, b_{1}\right) \in \mathbb{Z}^{n-1} \times\right. & \left(\frac{1}{2} \mathbb{Z}\right)^{2} \times \mathbb{Z}^{n-1} \mid \\
& \left.\sum_{i=1}^{n}\left(b_{i}+\bar{b}_{i}\right)=0, b_{n}+\bar{b}_{n} \in \mathbb{Z}\right\},
\end{aligned}
$$

for $b=\left(b_{1}, \cdots, b_{n}, \bar{b}_{n}, \cdots, b_{1}\right) \in B_{\infty}\left(B_{n}^{(1)}\right)$. We have

$$
\begin{aligned}
& \tilde{e}_{0} b= \begin{cases}\left(b_{1}, b_{2}-1, \cdots, \bar{b}_{2}, \bar{b}_{1}+1\right) & \text { if } b_{2}>\bar{b}_{2}, \\
\left(b_{1}-1, b_{2}, \cdots, \bar{b}_{2}+1, \bar{b}_{1}\right) & \text { if } b_{2} \leq \bar{b}_{2},\end{cases} \\
& \tilde{e}_{i} b=\left\{\begin{array}{ll}
\left(b_{1} \cdots, b_{i}+1, b_{i+1}-1, \cdots, \bar{b}_{1}\right) & \text { if } b_{i+1}>\bar{b}_{i+1}, \\
\left(b_{1} \cdots, \bar{b}_{i+1}+1, \bar{b}_{i}-1, \cdots, \bar{b}_{1}\right) & \text { if } b_{i+1} \leq \bar{b}_{i+1},
\end{array} \quad(i=1, \cdots, n-1),\right. \\
& \tilde{e}_{n} b=\left(b_{1}, \cdots, b_{n}+\frac{1}{2}, \bar{b}_{n}-\frac{1}{2}, \cdots, \bar{b}_{1}\right), \\
& \tilde{f}_{i}=\tilde{e}_{i}^{-1},
\end{aligned}
$$

and

$$
\left\{\begin{array}{l}
\operatorname{wt}(b)=\left(\bar{b}_{1}-b_{1}+\bar{b}_{2}-b_{2}\right) \Lambda_{0}+\sum_{i=1}^{n-1}\left(b_{i}-\bar{b}_{i}+\bar{b}_{i+1}-b_{i+1}\right) \Lambda_{i}+2\left(b_{n}-\bar{b}_{n}\right) \Lambda_{n}, \\
\varepsilon_{0}(b)=b_{1}+\left(b_{2}-\bar{b}_{2}\right)_{+}, \varepsilon_{i}(b)=\bar{b}_{i}+\left(b_{i+1}-\bar{b}_{i+1}\right)_{+}(i=1, \cdots, n-1), \\
\varepsilon_{n}(b)=2 \bar{b}_{n}, \varphi_{0}(b)=\bar{b}_{1}+\left(\bar{b}_{2}-b_{2}\right)_{+}, \varphi_{i}(b)=b_{i}+\left(\bar{b}_{i+1}-b_{i+1}\right)_{+}(i=1, \cdots, n-1), \\
\varphi_{n}(b)=2 b_{n}
\end{array}\right.
$$

Note that the presentation above is slightly different from the one in [6]. But we see that they are equivalent by the correspondence $\nu_{n}+\frac{1}{2} \nu_{0} \leftrightarrow b_{n}$ and $\bar{\nu}_{n}+\frac{1}{2} \nu_{0} \leftrightarrow \bar{b}_{n}$.

3.6. $\left.C_{n}^{(1)}\right)(n \geq 2)$. The Cartan matrix $\left(a_{i j}\right)_{i, j \in I}(I:=\{0,1, \cdots, n\})$ of type $C_{n}^{(1)}$ is

$$
a_{i j}= \begin{cases}2 & i=j \\ -1 & |i-j|=1 \text { and }(i, j) \neq(1,0),(n-1, n) \\ -2 & (i, j)=(1,0),(n-1, n) \\ 0 & \text { otherwise }\end{cases}
$$

Then the Dynkin diagram is

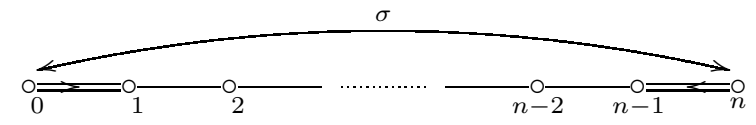

where $\sigma$ is the Dynkin diagram automorphism $\sigma: \alpha_{i} \mapsto \alpha_{n-i}$. We have

$$
\mathbf{c}=\sum_{i \in I} \alpha_{i}^{\vee}, \quad \delta=\alpha_{0}+2 \sum_{i=1}^{n-1} \alpha_{i}+\alpha_{n}
$$


A limit of perfect crystals is given as follows:

$$
B_{\infty}\left(C_{n}^{(1)}\right)=\left\{\left(b_{1}, \cdots, b_{n}, \bar{b}_{n}, \cdots, \bar{b}_{1}\right) \in \mathbb{Z}^{2 n} \mid \sum_{i=1}^{n}\left(b_{i}+\bar{b}_{i}\right) \in 2 \mathbb{Z}\right\}
$$

for $b=\left(b_{1}, \cdots, b_{n}, \bar{b}_{n}, \cdots, \bar{b}_{1}\right) \in B_{\infty}\left(C_{n}^{(1)}\right)$. We have

$$
\begin{aligned}
& \tilde{e}_{0} b= \begin{cases}\left(b_{1}-2, b_{2}, \cdots, \bar{b}_{2}, \bar{b}_{1}\right) & \text { if } b_{1}>\bar{b}_{1}+1, \\
\left(b_{1}-1, b_{2}, \cdots, \bar{b}_{2}, \bar{b}_{1}+1\right) & \text { if } b_{1}=\bar{b}_{1}+1, \\
\left(b_{1}, b_{2}, \cdots, \bar{b}_{2}, \bar{b}_{1}+2\right) & \text { if } b_{1} \leq \bar{b}_{1}\end{cases} \\
& \tilde{e}_{i} b=\left\{\begin{array}{ll}
\left(b_{1} \cdots, b_{i}+1, b_{i+1}-1, \cdots, \bar{b}_{1}\right) & \text { if } b_{i+1}>\bar{b}_{i+1}, \\
\left(b_{1} \cdots, \bar{b}_{i+1}+1, \bar{b}_{i}-1, \cdots, \bar{b}_{1}\right) & \text { if } b_{i+1} \leq \bar{b}_{i+1},
\end{array} \quad(1 \leq i<n),\right. \\
& \tilde{e}_{n} b=\left(b_{1}, \cdots, b_{n}+1, \bar{b}_{n}-1, \cdots, \bar{b}_{1}\right) \text {, } \\
& \tilde{f}_{i}=\tilde{e}_{i}^{-1} \text {, }
\end{aligned}
$$

and

$$
\left\{\begin{array}{l}
\operatorname{wt}(b)=\left(\bar{b}_{1}-b_{1}\right) \Lambda_{0}+\sum_{i=1}^{n-1}\left(b_{i}-\bar{b}_{i}+\bar{b}_{i+1}-b_{i+1}\right) \Lambda_{i}+\left(b_{n}-\bar{b}_{n}\right) \Lambda_{n}, \\
\varepsilon_{0}(b)=-\frac{1}{2} l(b)+\left(b_{1}-\bar{b}_{1}\right)_{+}, \varepsilon_{i}(b)=\bar{b}_{i}+\left(b_{i+1}-\bar{b}_{i+1}\right)_{+}(1 \leq i<n), \varepsilon_{n}(b)=\bar{b}_{n}, \\
\varphi_{0}(b)=-\frac{1}{2} l(b)+\left(\bar{b}_{1}-b_{1}\right)_{+}, \varphi_{i}(b)=b_{i}+\left(\bar{b}_{i+1}-b_{i+1}\right)_{+}(1 \leq i<n), \varphi_{n}(b)=b_{n},
\end{array}\right.
$$

where $l(b):=\sum_{i=1}^{n}\left(b_{i}+\bar{b}_{i}\right)$.

3.7. $D_{n}^{(1)}(n \geq 4)$. The Cartan matrix $\left(a_{i j}\right)_{i, j \in I}(I:=\{0,1, \cdots, n\})$ of type $D_{n}^{(1)}$ is

$$
a_{i j}= \begin{cases}2 & i=j \\ -1 & |i-j|=1 \text { and } 1 \leq i, j \leq n-1 \\ & \text { or }(i, j)=(0,2),(2,0),(n-2, n),(n, n-2) \\ 0 \quad & \text { otherwise }\end{cases}
$$

The Dynkin diagram is

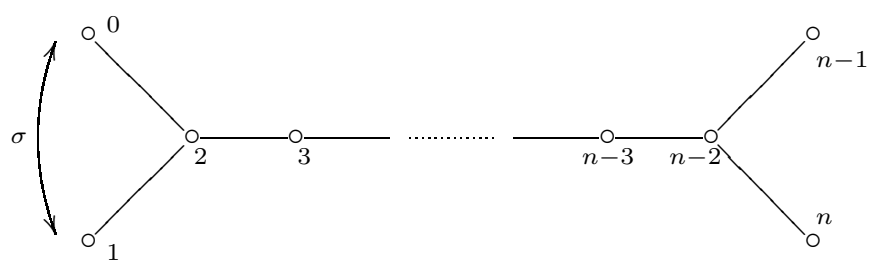

where $\sigma$ is the Dynkin diagram automorphism $\sigma: \alpha_{0} \leftrightarrow \alpha_{1}$ and $\sigma \alpha_{i}=\alpha_{i}$ for $i \neq 0,1$. We have

$$
\mathbf{c}=\alpha_{0}^{\vee}+\alpha_{1}^{\vee}+2 \sum_{i=2}^{n-2} \alpha_{i}^{\vee}+\alpha_{n-1}^{\vee}+\alpha_{n}^{\vee}, \quad \delta=\alpha_{0}+\alpha_{1}+2 \sum_{i=2}^{n-2} \alpha_{i}+\alpha_{n-1}+\alpha_{n} .
$$


A limit of perfect crystals is given as follows:

$$
B_{\infty}\left(D_{n}^{(1)}\right)=\left\{\left(b_{1}, \cdots, b_{n}, \bar{b}_{n-1}, \cdots, \bar{b}_{1}\right) \in \mathbb{Z}^{2 n-1} \mid \sum_{i=1}^{n} b_{i}+\sum_{1}^{n-1} \bar{b}_{i}=0\right\},
$$

for $b=\left(b_{1}, \cdots, b_{n}, \bar{b}_{n-1}, \cdots, \bar{b}_{1}\right) \in B_{\infty}\left(D_{n}^{(1)}\right)$. We have

$$
\begin{aligned}
& \tilde{e}_{0} b= \begin{cases}\left(b_{1}, b_{2}-1, \cdots, \bar{b}_{2}, \bar{b}_{1}+1\right) & \text { if } b_{2}>\bar{b}_{2}, \\
\left(b_{1}-1, b_{2}, \cdots, \bar{b}_{2}+1, \bar{b}_{1}\right) & \text { if } b_{2} \leq \bar{b}_{2},\end{cases} \\
& \tilde{e}_{i} b=\left\{\begin{array}{ll}
\left(b_{1} \cdots, b_{i}+1, b_{i+1}-1, \cdots, \bar{b}_{1}\right) & \text { if } b_{i+1}>\bar{b}_{i+1}, \\
\left(b_{1} \cdots, \bar{b}_{i+1}+1, \bar{b}_{i}-1, \cdots, \bar{b}_{1}\right) & \text { if } b_{i+1} \leq \bar{b}_{i+1},
\end{array}(i=1, \cdots, n-2),\right. \\
& \tilde{e}_{n-1} b=\left(b_{1}, \cdots, b_{n-1}+1, b_{n}-1, \bar{b}_{n-1}, \cdots, \bar{b}_{1}\right), \\
& \tilde{e}_{n} b=\left(b_{1}, \cdots, b_{n-1}, b_{n}+1, \bar{b}_{n-1}-1 \cdots, \bar{b}_{1}\right), \\
& \tilde{f}_{i}=\tilde{e}_{i}^{-1},
\end{aligned}
$$

and

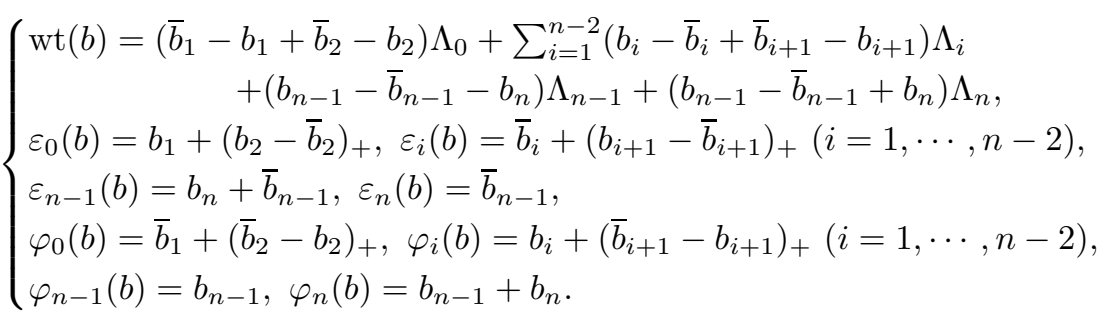

3.8. $A_{2 n-1}^{(2)}(n \geq 3)$. The Cartan matrix $\left(a_{i j}\right)_{i, j \in I}(I:=\{0,1, \cdots, n\})$ of type $A_{2 n-1}^{(2)}$ is

$$
a_{i j}= \begin{cases}2 & i=j, \\ -1 & |i-j|=1 \text { and } 1 \leq i, j \leq n-1 \text { or }(i, j)=(0,2),(2,0),(n, n-1), \\ -2 & (i, j)=(n-1, n), \\ 0 & \text { otherwise. }\end{cases}
$$

The Dynkin diagram is

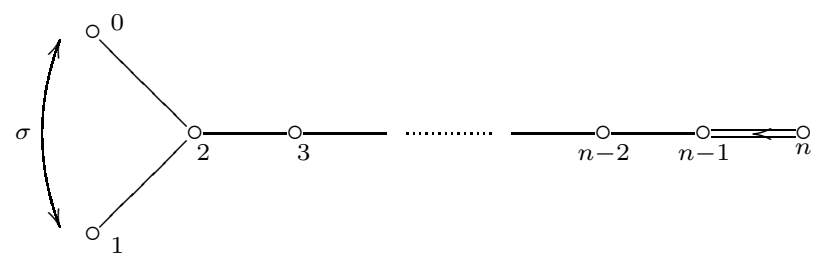

where $\sigma$ is the Dynkin diagram automorphism $\sigma: \alpha_{0} \leftrightarrow \alpha_{1}$. We have

$$
\mathbf{c}=\alpha_{0}^{\vee}+\alpha_{1}^{\vee}+2 \sum_{i=2}^{n} \alpha_{i}^{\vee}, \quad \delta=\alpha_{0}+\alpha_{1}+2 \sum_{i=2}^{n-1} \alpha_{i}+\alpha_{n} .
$$

A limit of perfect crystals is given as follows:

$$
B_{\infty}\left(A_{2 n-1}^{(2)}\right)=\left\{\left(b_{1}, \cdots, b_{n}, \bar{b}_{n}, \cdots, \bar{b}_{1}\right) \in \mathbb{Z}^{2 n} \mid \sum_{i=1}^{n}\left(b_{i}+\bar{b}_{i}\right)=0\right\},
$$


for $b=\left(b_{1}, \cdots, b_{n}, \bar{b}_{n}, \cdots, \bar{b}_{1}\right) \in B_{\infty}\left(A_{2 n-1}^{(2)}\right)$. We have

$$
\begin{aligned}
& \tilde{e}_{0} b= \begin{cases}\left(b_{1}, b_{2}-1, \cdots, \bar{b}_{2}, \bar{b}_{1}+1\right) & \text { if } b_{2}>\bar{b}_{2}, \\
\left(b_{1}-1, b_{2}, \cdots, \bar{b}_{2}+1, \bar{b}_{1}\right) & \text { if } b_{2} \leq \bar{b}_{2},\end{cases} \\
& \tilde{e}_{i} b=\left\{\begin{array}{ll}
\left(b_{1} \cdots, b_{i}+1, b_{i+1}-1, \cdots, \bar{b}_{1}\right) & \text { if } b_{i+1}>\bar{b}_{i+1}, \\
\left(b_{1} \cdots, \bar{b}_{i+1}+1, \bar{b}_{i}-1, \cdots, \bar{b}_{1}\right) & \text { if } b_{i+1} \leq \bar{b}_{i+1},
\end{array},(i=1, \cdots, n-1),\right. \\
& \tilde{e}_{n} b=\left(b_{1}, \cdots, b_{n-1}, b_{n}+1, \bar{b}_{n}-1, \cdots, \bar{b}_{1}\right), \\
& \tilde{f}_{i}=\tilde{e}_{i}^{-1},
\end{aligned}
$$

and

$$
\left\{\begin{array}{l}
\operatorname{wt}(b)=\left(\bar{b}_{1}-b_{1}+\bar{b}_{2}-b_{2}\right) \Lambda_{0}+\sum_{i=1}^{n-1}\left(b_{i}-\bar{b}_{i}+\bar{b}_{i+1}-b_{i+1}\right) \Lambda_{i}+\left(b_{n}-\bar{b}_{n}\right) \Lambda_{n} \\
\varepsilon_{0}(b)=b_{1}+\left(b_{2}-\bar{b}_{2}\right)_{+}, \varepsilon_{i}(b)=\bar{b}_{i}+\left(b_{i+1}-\bar{b}_{i+1}\right)_{+}(i=1, \cdots, n-1) \\
\varepsilon_{n}(b)=\bar{b}_{n}, \varphi_{0}(b)=\bar{b}_{1}+\left(\bar{b}_{2}-b_{2}\right)_{+}, \varphi_{i}(b)=b_{i}+\left(\bar{b}_{i+1}-b_{i+1}\right)_{+}(i=1, \cdots, n-1) \\
\varphi_{n}(b)=b_{n}
\end{array}\right.
$$

3.9. $D_{n+1}^{(2)}(n \geq 2)$. The Cartan matrix $\left(a_{i j}\right)_{i, j \in I}(I:=\{0,1, \cdots, n\})$ of type $D_{n+1}^{(2)}$ is

$$
a_{i j}= \begin{cases}2 & i=j \\ -1 & |i-j|=1 \text { and }(i, j) \neq(0,1),(n, n-1), \\ -2 & (i, j)=(0,1),(n, n-1), \\ 0 & \text { otherwise. }\end{cases}
$$

The Dynkin diagram is

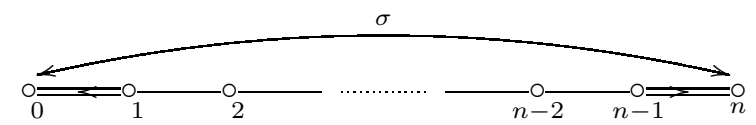

where $\sigma$ is the Dynkin diagram automorphism $\sigma: \alpha_{i} \leftrightarrow \alpha_{n-i}(i=0,1 \cdots, n)$. We have

$$
\mathbf{c}=\alpha_{0}^{\vee}+\alpha_{1}^{\vee}+2 \sum_{i=2}^{n-1} \alpha_{i}^{\vee}+\alpha_{n}^{\vee}, \quad \delta=\sum_{i=0}^{n} \alpha_{i} .
$$

A limit of perfect crystals is given as follows:

$$
B_{\infty}\left(D_{n+1}^{(2)}\right)=\left\{\left(b_{1}, \cdots, b_{n}, \bar{b}_{n}, \cdots, b_{1}\right) \in \mathbb{Z}^{n-1} \times\left(\frac{1}{2} \mathbb{Z}\right)^{2} \times \mathbb{Z}^{n-1} \mid b_{n}+\bar{b}_{n} \in \mathbb{Z}\right\},
$$

for $b=\left(b_{1}, \cdots, b_{n}, \bar{b}_{n}, \cdots, b_{1}\right) \in B_{\infty}\left(D_{n+1}^{(2)}\right)$. We have

$$
\begin{aligned}
& \tilde{e}_{0} b=\left\{\begin{array}{l}
\left(b_{1}-1, b_{2}, \cdots, \bar{b}_{2}, \bar{b}_{1}\right) \quad \text { if } b_{1}>\bar{b}_{1}, \\
\left(b_{1}, b_{2}, \cdots, \bar{b}_{2}, \bar{b}_{1}+1\right) \quad \text { if } b_{1} \leq \bar{b}_{1},
\end{array}\right. \\
& \tilde{e}_{i} b=\left\{\begin{array}{l}
\left(b_{1} \cdots, b_{i}+1, b_{i+1}-1, \cdots, \bar{b}_{1}\right) \quad \text { if } b_{i+1}>\bar{b}_{i+1}, \\
\left(b_{1} \cdots, \bar{b}_{i+1}+1, \bar{b}_{i}-1, \cdots, \bar{b}_{1}\right) \quad \text { if } b_{i+1} \leq \bar{b}_{i+1},
\end{array}(1 \leq i<n),\right. \\
& \tilde{e}_{n} b=\left(b_{1}, \cdots, b_{n}+\frac{1}{2}, \bar{b}_{n}-\frac{1}{2}, \cdots, \bar{b}_{1}\right), \\
& \tilde{f}_{i}=\tilde{e}_{i}^{-1},
\end{aligned}
$$


and

$$
\left\{\begin{array}{l}
\operatorname{wt}(b)=2\left(\bar{b}_{1}-b_{1}\right) \Lambda_{0}+\sum_{i=1}^{n-1}\left(b_{i}-\bar{b}_{i}+\bar{b}_{i+1}-b_{i+1}\right) \Lambda_{i}+2\left(b_{n}-\bar{b}_{n}\right) \Lambda_{n} \\
\varepsilon_{0}(b)=-l(b)+2\left(b_{1}-\bar{b}_{1}\right)_{+}, \varepsilon_{i}(b)=\bar{b}_{i}+\left(b_{i+1}-\bar{b}_{i+1}\right)_{+}(1 \leq i<n), \varepsilon_{n}(b)=2 \bar{b}_{n} \\
\varphi_{0}(b)=-l(b)+2\left(\bar{b}_{1}-b_{1}\right)_{+}, \varphi_{i}(b)=b_{i}+\left(\bar{b}_{i+1}-b_{i+1}\right)_{+}(1 \leq i<n), \varphi_{n}(b)=2 b_{n}
\end{array}\right.
$$

where $l(b):=\sum_{i=1}^{n}\left(b_{i}+\bar{b}_{i}\right)$. Note that the presentation above is slightly different from the one in [6]. As in 93.5 , they are equivalent by the correspondence $\nu_{n}+\frac{1}{2} \nu_{0} \leftrightarrow b_{n}$ and $\bar{\nu}_{n}+\frac{1}{2} \nu_{0} \leftrightarrow \bar{b}_{n}$.

3.10. $A_{2 n}^{(2)}(n \geq 2)$. The Cartan matrix $\left(a_{i j}\right)_{i, j \in I}(I:=\{0,1, \cdots, n\})$ of type $A_{2 n}^{(2)}$ is

$$
a_{i j}= \begin{cases}2 & i=j, \\ -1 & |i-j|=1 \text { and }(i, j) \neq(0,1),(n-1, n), \\ -2 & (i, j)=(0,1),(n-1, n), \\ 0 & \text { otherwise }\end{cases}
$$

The Dynkin diagram is

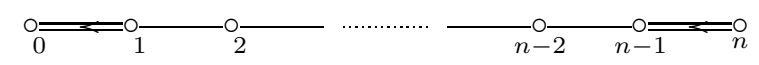

Note that there exists no Dynkin diagram automorphism in this case. We have

$$
\mathbf{c}=\alpha_{0}^{\vee}+2 \sum_{i=1}^{n} \alpha_{i}^{\vee}, \quad \delta=2 \sum_{i=0}^{n-1} \alpha_{i}+\alpha_{n} .
$$

A limit of perfect crystals is given as follows:

$$
B_{\infty}\left(A_{2 n}^{(2)}\right)=\left\{\left(b_{1}, \cdots, b_{n}, \bar{b}_{n}, \cdots, \bar{b}_{1}\right) \in \mathbb{Z}^{2 n}\right\}
$$

for $\left(b_{1}, \cdots, b_{n}, \bar{b}_{n}, \cdots, \bar{b}_{1}\right) \in B_{\infty}\left(A_{2 n}^{(2)}\right)$. We have

$$
\begin{aligned}
& \tilde{e}_{0} b= \begin{cases}\left(b_{1}-1, b_{2}, \cdots, \bar{b}_{2}, \bar{b}_{1}\right) & \text { if } b_{1}>\bar{b}_{1}, \\
\left(b_{1}, b_{2}, \cdots, \bar{b}_{2}, \bar{b}_{1}+1\right) & \text { if } b_{1} \leq \bar{b}_{1},\end{cases} \\
& \tilde{e}_{i} b=\left\{\begin{array}{ll}
\left(b_{1} \cdots, b_{i}+1, b_{i+1}-1, \cdots, \bar{b}_{1}\right) & \text { if } b_{i+1}>\bar{b}_{i+1}, \\
\left(b_{1} \cdots, \bar{b}_{i+1}+1, \bar{b}_{i}-1, \cdots, \bar{b}_{1}\right) & \text { if } b_{i+1} \leq \bar{b}_{i+1},
\end{array} \quad(1 \leq i<n),\right. \\
& \tilde{e}_{n} b=\left(b_{1}, \cdots, b_{n-1}, b_{n}+1, \bar{b}_{n}-1, \cdots, \bar{b}_{1}\right) \text {, } \\
& \tilde{f}_{i}=\tilde{e}_{i}^{-1} \text {, }
\end{aligned}
$$

and

$$
\left\{\begin{array}{l}
\mathrm{wt}(b)=2\left(\bar{b}_{1}-b_{1}\right) \Lambda_{0}+\sum_{i=1}^{n-1}\left(b_{i}-\bar{b}_{i}+\bar{b}_{i+1}-b_{i+1}\right) \Lambda_{i}+\left(b_{n}-\bar{b}_{n}\right) \Lambda_{n}, \\
\varepsilon_{0}(b)=-l(b)+2\left(b_{1}-\bar{b}_{1}\right)_{+}, \varepsilon_{i}(b)=\bar{b}_{i}+\left(b_{i+1}-\bar{b}_{i+1}\right)_{+}(1 \leq i<n), \varepsilon_{n}(b)=\bar{b}_{n}, \\
\varphi_{0}(b)=-l(b)+2\left(\bar{b}_{1}-b_{1}\right)_{+}, \varphi_{i}(b)=b_{i}+\left(\bar{b}_{i+1}-b_{i+1}\right)_{+}(1 \leq i<n), \varphi_{n}(b)=b_{n},
\end{array}\right.
$$

where $l(b):=\sum_{i=1}^{n}\left(b_{i}+\bar{b}_{i}\right)$. 


\section{Fundamental Representations}

4.1. Fundamental representation $W\left(\varpi_{1}\right)$. Let $\mathfrak{g}=A_{n}^{(1)}, B_{n}^{(1)}, C_{n}^{(1)}, D_{n}^{(1)}$, $A_{2 n-1}^{(2)}, D_{n+1}^{(2)}, A_{2 n}^{(2)}$, and let $\left\{\Lambda_{i} \mid i \in I\right\}$ be the set of fundamental weights as in \$3. Let $\varpi_{1}:=\Lambda_{1}-a_{1}^{\vee} \Lambda_{0}$ be the (level 0 ) fundamental weight, where $i=1$ is the node of the Dynkin diagram as in $\$ 3$ and $a_{i}^{\vee}$ is given in (3.1).

Let $V\left(\varpi_{1}\right)$ be the extremal weight module over $U_{q}(\mathfrak{g})$ associated with $\varpi_{1}([9])$ and let $W\left(\varpi_{1}\right) \cong V\left(\varpi_{1}\right) /\left(z_{1}-1\right) V\left(\varpi_{1}\right)$ be the fundamental representation of $U_{q}^{\prime}(\mathfrak{g})$ where $z_{1}$ is a $U_{q}^{\prime}(\mathfrak{g})$-linear automorphism on $V\left(\varpi_{1}\right)$ (see [9, Sect. 5]).

By [9, Theorem 5.17], $W\left(\varpi_{1}\right)$ is a finite-dimensional irreducible integrable $U_{q}^{\prime}(\mathfrak{g})$ module and has a global basis with a simple crystal. Thus, we can consider its specialization $q=1$ and obtain a finite-dimensional $\mathfrak{g}$-module, which will be denoted by the same notation $W\left(\varpi_{1}\right)$.

Now we present the explicit form of $W\left(\varpi_{1}\right)$ for $\mathfrak{g}=A_{n}^{(1)}, B_{n}^{(1)}, C_{n}^{(1)}, D_{n}^{(1)}, A_{2 n-1}^{(2)}$, $D_{n+1}^{(2)}, A_{2 n}^{(2)}$.

4.2. $A_{n}^{(1)}(n \geq 2)$. The global basis of $W\left(\varpi_{1}\right)$ is

$$
\left\{v_{1}, v_{2}, \cdots, v_{n+1}\right\},
$$

and we have

$$
\operatorname{wt}\left(v_{i}\right)=\Lambda_{i}-\Lambda_{i-1} \quad(i=1 \cdots, n+1),
$$

where we understand $\Lambda_{n+1}=\Lambda_{0}$. The explicit actions of the $f_{i}$ 's are

$$
\begin{array}{ll}
f_{i} v_{i}=v_{i+1} & (1 \leq i \leq n), \quad f_{0} v_{n+1}=v_{1} \\
f_{i} v_{j}=0 & \text { otherwise }
\end{array}
$$

Its crystal graph is:

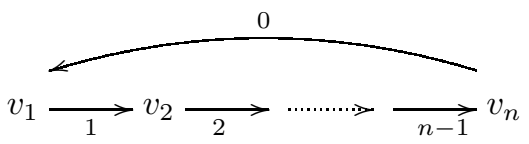

4.3. $B_{n}^{(1)}(n \geq 3)$. The global basis of $W\left(\varpi_{1}\right)$ is

$$
\left\{v_{1}, v_{2}, \cdots, v_{n}, v_{0}, v_{\bar{n}}, \cdots, v_{\overline{2}}, v_{\overline{1}}\right\},
$$

and we have

$$
\begin{aligned}
& \operatorname{wt}\left(v_{i}\right)=\Lambda_{i}-\Lambda_{i-1}, \quad \quad \operatorname{wt}\left(v_{\bar{i}}\right)=\Lambda_{i-1}-\Lambda_{i} \quad(i \neq 0,2, n), \\
& \operatorname{wt}\left(v_{2}\right)=-\Lambda_{0}-\Lambda_{1}+\Lambda_{2}, \quad \operatorname{wt}\left(v_{\overline{2}}\right)=\Lambda_{0}+\Lambda_{1}-\Lambda_{2}, \\
& \operatorname{wt}\left(v_{n}\right)=2 \Lambda_{n}-\Lambda_{n-1}, \quad \operatorname{wt}\left(v_{\bar{n}}\right)=\Lambda_{n-1}-2 \Lambda_{n}, \quad \operatorname{wt}\left(v_{0}\right)=0 .
\end{aligned}
$$

The explicit forms of the actions by the $f_{i}$ 's are

$$
\begin{aligned}
& f_{i} v_{i}=v_{i+1}, \quad f_{i} v_{\overline{i+1}}=v_{\bar{i}} \quad(i=1, \cdots, n-1), \\
& f_{n} v_{n}=v_{0}, \quad f_{n} v_{0}=2 v_{\bar{n}}, \\
& f_{0} v_{\overline{2}}=v_{1}, \quad f_{0} v_{\overline{1}}=v_{2}, \\
& f_{i} v_{j}=0 \quad \text { otherwise. }
\end{aligned}
$$


Its crystal graph is

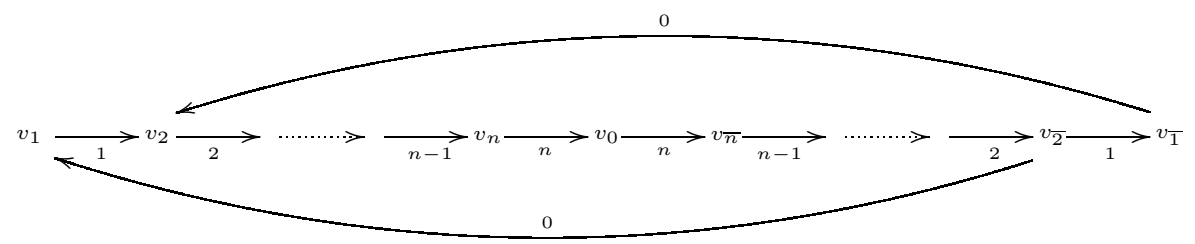

4.4. $C_{n}^{(1)}(n \geq 2)$. The global basis of $W\left(\varpi_{1}\right)$ is

$$
\left\{v_{1}, v_{2}, \cdots, v_{n}, v_{\bar{n}}, \cdots, v_{\overline{2}}, v_{\overline{1}}\right\},
$$

and we have

$$
\operatorname{wt}\left(v_{i}\right)=\Lambda_{i}-\Lambda_{i-1}, \quad \operatorname{wt}\left(v_{\bar{i}}\right)=\Lambda_{i-1}-\Lambda_{i} \quad(i=1 \cdots, n) .
$$

The explicit forms of the actions by the $f_{i}$ 's are

$$
\begin{aligned}
& f_{i} v_{i}=v_{i+1}, \quad f_{i} v_{\overline{i+1}}=v_{\bar{i}} \quad(i=1, \cdots, n-1), \\
& f_{n} v_{n}=v_{\bar{n}}, \quad f_{0} v_{\overline{1}}=v_{1}, \\
& f_{i} v_{j}=0 \quad \text { otherwise. }
\end{aligned}
$$

Its crystal graph is

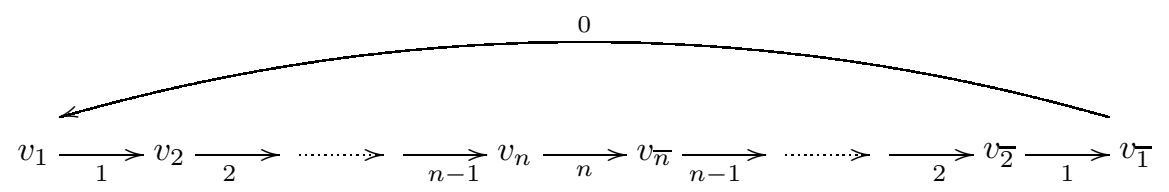

4.5. $D_{n}^{(1)}(n \geq 4)$. The global basis of $W\left(\varpi_{1}\right)$ is

$$
\left\{v_{1}, v_{2}, \cdots, v_{n}, v_{\bar{n}}, \cdots, v_{\overline{2}}, v_{\overline{1}}\right\},
$$

and we have

$$
\begin{aligned}
& \operatorname{wt}\left(v_{i}\right)=\Lambda_{i}-\Lambda_{i-1}, \quad \operatorname{wt}\left(v_{\bar{i}}\right)=\Lambda_{i-1}-\Lambda_{i} \quad(i \neq 2, n-1), \\
& \operatorname{wt}\left(v_{2}\right)=-\Lambda_{0}-\Lambda_{1}+\Lambda_{2}, \quad \operatorname{wt}\left(v_{\overline{2}}\right)=\Lambda_{0}+\Lambda_{1}-\Lambda_{2}, \\
& \operatorname{wt}\left(v_{n-1}\right)=\Lambda_{n-1}+\Lambda_{n}-\Lambda_{n-1}, \quad \operatorname{wt}\left(v_{\overline{n-1}}\right)=\Lambda_{n-2}-\Lambda_{n-1}-\Lambda_{n} .
\end{aligned}
$$

The explicit forms of the actions by the $f_{i}$ 's are

$$
\begin{aligned}
& f_{i} v_{i}=v_{i+1}, \quad f_{i} v_{\overline{i+1}}=v_{\bar{i}} \quad(i=1, \cdots, n-1), \\
& f_{n} v_{n}=v_{\overline{n-1}}, \quad f_{n} v_{n-1}=v_{\bar{n}}, \\
& f_{0} v_{\overline{2}}=v_{1}, \quad f_{0} v_{\overline{1}}=v_{2}, \\
& f_{i} v_{j}=0 \quad \text { otherwise. }
\end{aligned}
$$


Its crystal graph is

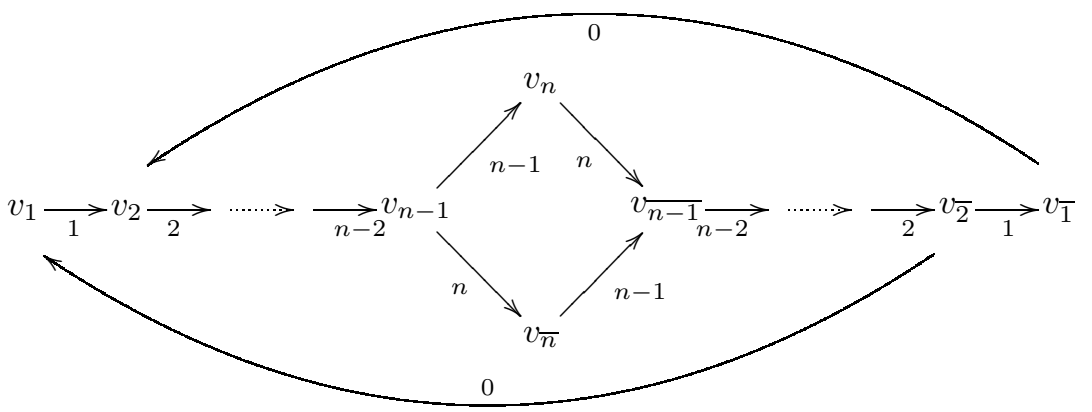

4.6. $A_{2 n-1}^{(2)}(n \geq 3)$. The global basis of $W\left(\varpi_{1}\right)$ is

$$
\left\{v_{1}, v_{2}, \cdots, v_{n}, v_{\bar{n}}, \cdots, v_{\overline{2}}, v_{\overline{1}}\right\} \text {, }
$$

and we have

$$
\begin{aligned}
& \operatorname{wt}\left(v_{i}\right)=\Lambda_{i}-\Lambda_{i-1}, \quad \operatorname{wt}\left(v_{\bar{i}}\right)=\Lambda_{i-1}-\Lambda_{i} \quad(i \neq 2), \\
& \operatorname{wt}\left(v_{2}\right)=-\Lambda_{0}-\Lambda_{1}+\Lambda_{2}, \quad \operatorname{wt}\left(v_{\overline{2}}\right)=\Lambda_{0}+\Lambda_{1}-\Lambda_{2} .
\end{aligned}
$$

The explicit forms of the actions by the $f_{i}$ 's are

$$
\begin{aligned}
& f_{i} v_{i}=v_{i+1}, \quad f_{i} v_{\overline{i+1}}=v_{\bar{i}} \quad(i=1, \cdots, n-1), \\
& f_{n} v_{n}=v_{\bar{n}}, \\
& f_{0} v_{\overline{2}}=v_{1}, \quad f_{0} v_{\overline{1}}=v_{2}, \\
& f_{i} v_{j}=0 \quad \text { otherwise. }
\end{aligned}
$$

Its crystal graph is

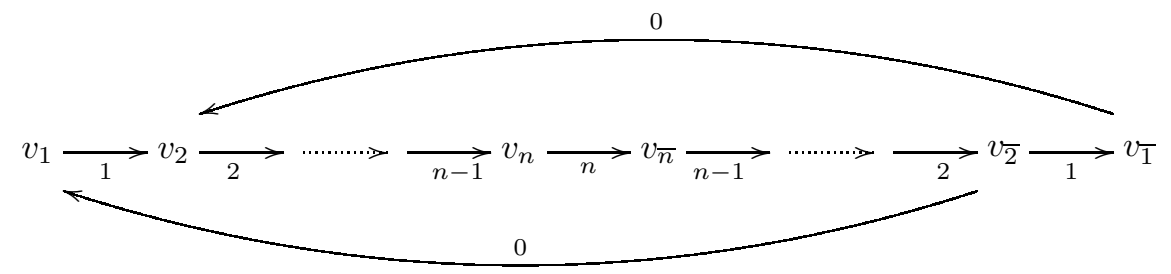

4.7. $D_{n+1}^{(2)}(n \geq 2)$. The global basis of $W\left(\varpi_{1}\right)$ is

$$
\left\{v_{1}, v_{2}, \cdots, v_{n}, v_{0}, v_{\bar{n}}, \cdots, v_{\overline{2}}, v_{\overline{1}}, \phi\right\} \text {, }
$$

and we have

$$
\begin{aligned}
& \operatorname{wt}\left(v_{i}\right)=\Lambda_{i}-\Lambda_{i-1}, \quad \quad \operatorname{wt}\left(v_{\bar{i}}\right)=\Lambda_{i-1}-\Lambda_{i} \quad(i \neq 0,1, n), \\
& \operatorname{wt}\left(v_{1}\right)=\Lambda_{1}-2 \Lambda_{0}, \quad \operatorname{wt}\left(v_{\overline{1}}\right)=2 \Lambda_{0}-\Lambda_{1}, \\
& \operatorname{wt}\left(v_{n}\right)=2 \Lambda_{n}-\Lambda_{n-1}, \quad \operatorname{wt}\left(v_{\bar{n}}\right)=\Lambda_{n-1}-2 \Lambda_{n}, \\
& \operatorname{wt}\left(v_{0}\right)=0, \quad \operatorname{wt}(\phi)=0 .
\end{aligned}
$$

The explicit forms of the actions by the $f_{i}$ 's are

$$
\begin{aligned}
& f_{i} v_{i}=v_{i+1}, \quad f_{i} v_{\overline{i+1}}=v_{\bar{i}} \quad(i=1, \cdots, n-1), \\
& f_{n} v_{n}=v_{0}, \quad f_{n} v_{0}=2 v_{\bar{n}}, \\
& f_{0} v_{\overline{1}}=\phi, \quad f_{0} \phi=2 v_{1}, \\
& f_{i} v_{j}=0, \quad f_{i} \phi=0 \quad \text { otherwise. }
\end{aligned}
$$


Its crystal graph is

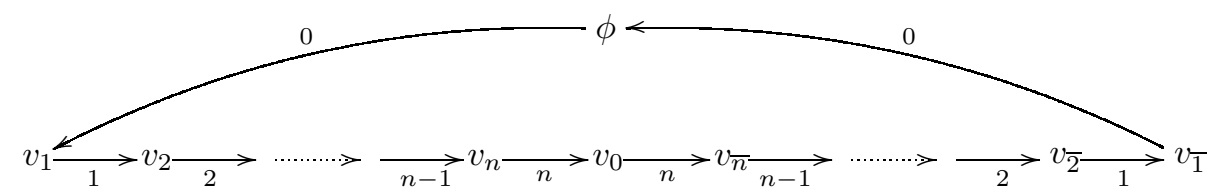

4.8. $A_{2 n}^{(2) \dagger}(n \geq 2)$. We take the Cartan data transposed from that in $₫ 3.10$. Then the Dynkin diagram is

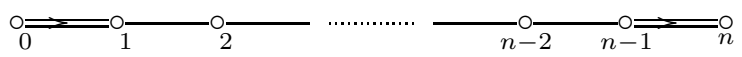

In this case, we denote this type by $A_{2 n}^{(2) \dagger}$ in order to distinguish it from the one in 3.10 . Then the global basis of $W\left(\varpi_{1}\right)$ is

$$
\left\{v_{1}, v_{2}, \cdots, v_{n}, v_{0}, v_{\bar{n}}, \cdots, v_{\overline{2}}, v_{\overline{1}}\right\} \text {, }
$$

and we have

$$
\begin{aligned}
& \operatorname{wt}\left(v_{i}\right)=\Lambda_{i}-\Lambda_{i-1}, \quad \operatorname{wt}\left(v_{\bar{i}}\right)=\Lambda_{i-1}-\Lambda_{i} \quad(i=1, \cdots, n-1), \\
& \operatorname{wt}\left(v_{n}\right)=2 \Lambda_{n}-\Lambda_{n-1}, \quad \operatorname{wt}\left(v_{0}\right)=0, \quad \operatorname{wt}\left(v_{\bar{n}}\right)=\Lambda_{n-1}-2 \Lambda_{n} .
\end{aligned}
$$

The explicit forms of the actions by the $f_{i}$ 's are

$$
\begin{aligned}
& f_{i} v_{i}=v_{i+1}, \quad f_{i} v_{\overline{i+1}}=v_{\bar{i}} \quad(i=1, \cdots, n-1), \\
& f_{n} v_{n}=v_{\overline{0}}, \quad f_{n} v_{0}=2 v_{\bar{n}}, \\
& f_{0} v_{\overline{1}}=v_{1}, \\
& f_{i} v_{j}=0, \quad \text { otherwise. }
\end{aligned}
$$

Its crystal graph is

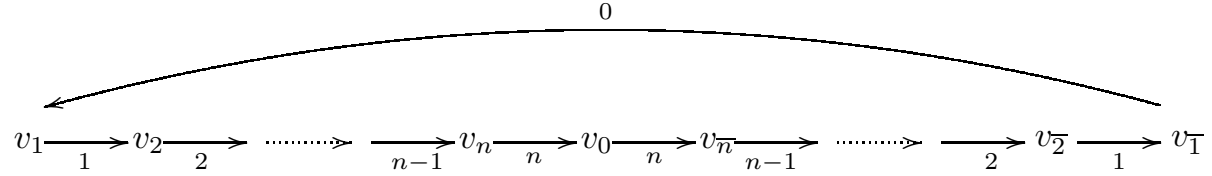

\section{Affine geometric CRystals}

In this section, we shall construct the affine geometric crystal $\mathcal{V}(\mathfrak{g})$, which is realized in the fundamental representation $W\left(\varpi_{1}\right)$.

5.1. Translation $t\left(\tilde{\varpi}_{1}\right)$. For $\xi_{0} \in\left(\mathfrak{t}_{\mathrm{cl}}^{*}\right)_{0}$, let $t\left(\xi_{0}\right)$ be as in [9, Sect.4]:

$$
t\left(\xi_{0}\right)(\lambda):=\lambda+(\delta, \lambda) \xi-(\xi, \lambda) \delta-\frac{(\xi, \xi)}{2}(\delta, \lambda) \delta
$$


for $\xi \in \mathfrak{t}^{*}$ such that $\operatorname{cl}(\xi)=\xi_{0}$. Then $t\left(\xi_{0}\right)$ does not depend on the choice of $\xi$, and it is well-defined.

Let $c_{i}^{\vee}$ be as in (1.1). Then $t\left(m \varpi_{i}\right)$ belongs to $\widetilde{W}$ if and only if $m \in c_{i}^{\vee} \mathbb{Z}$. Setting $\widetilde{\varpi}_{i}:=c_{i}^{\vee} \varpi_{i}(i \in I)([10]), t\left(\widetilde{\varpi}_{1}\right)$ is expressed as follows (see e.g. [12]):

$$
t\left(\widetilde{\varpi}_{1}\right)= \begin{cases}\iota\left(s_{n} s_{n-1} \cdots s_{2} s_{1}\right) & A_{n}^{(1)} \text { case, } \\ \iota\left(s_{1} \cdots s_{n}\right)\left(s_{n-1} \cdots s_{2} s_{1}\right) & B_{n}^{(1)}, A_{2 n-1}^{(2)} \text { cases, } \\ \left(s_{0} s_{1} \cdots s_{n}\right)\left(s_{n-1} \cdots s_{2} s_{1}\right) & C_{n}^{(1)}, D_{n+1}^{(2)} \text { cases, } \\ \iota\left(s_{1} \cdots s_{n}\right)\left(s_{n-2} \cdots s_{2} s_{1}\right) & D_{n}^{(1)} \text { case, } \\ \left(s_{0} s_{1} \cdots s_{n}\right)\left(s_{n-1} \cdots s_{2} s_{1}\right) & A_{2 n}^{(2) \dagger} \text { case, }\end{cases}
$$

where $\iota$ is the Dynkin diagram automorphism

$$
\iota= \begin{cases}\sigma & \mathfrak{g}=A_{n}^{(1)}, B_{n}^{(1)}, A_{2 n-1}^{(2)}, \\ \alpha_{0} \leftrightarrow \alpha_{1} \text { and } \alpha_{n-1} \leftrightarrow \alpha_{n} & \mathfrak{g}=D_{n}^{(1)} .\end{cases}
$$

Now, we know that each $t\left(\widetilde{\varpi}_{1}\right)$ is in the form $w_{1}$ or $\iota \cdot w_{1}$ for some $w_{1} \in W$, e.g., $w_{1}=s_{n} \cdots s_{1}$ for $A_{n}^{(1)}, w_{1}=\left(s_{1} \cdots s_{n}\right)\left(s_{n-1} \cdots s_{1}\right)$ for $B_{n}^{(1)}$, etc.,$\ldots$

In the case $\mathfrak{g}=A_{2 n}^{(2) \dagger}, \eta:=\operatorname{wt}\left(v_{\bar{n}}\right)=\Lambda_{n-1}-2 \Lambda_{n}$ is a unique weight of $W\left(\varpi_{1}\right)$ which satisfies $\left\langle\alpha_{i}^{\vee}, \eta\right\rangle \geq 0$ for $i \neq n$. For this $\eta$ we have

$$
t(\eta)=\left(s_{n} s_{n-1} \cdots s_{1}\right)\left(s_{0} s_{1} \cdots s_{n-1}\right)=: w_{2},
$$

which will be used later.

5.2. Affine geometric crystals. Let $\sigma$ be the Dynkin diagram automorphism as in 33.43 .9 and $w_{1}=s_{i_{1}} \cdots s_{i_{k}}$ be as in the previous subsection. Set

$$
\mathcal{V}(\mathfrak{g}):=\left\{v\left(x_{1}, \cdots, x_{k}\right):=Y_{i_{1}}\left(x_{1}\right) \cdots Y_{i_{k}}\left(x_{k}\right) v_{1} \mid x_{1}, \cdots, x_{k} \in \mathbb{C}^{\times}\right\} \subset W\left(\varpi_{1}\right) .
$$

Since the vector $v_{1}$ is a highest weight vector in $W\left(\varpi_{1}\right)$ as a $\mathfrak{g}_{0}$-module, $\mathcal{V}(\mathfrak{g})$ has a $G_{0}$-geometric crystal structure. Moreover $\left(\mathbb{C}^{\times}\right)^{k} \rightarrow \mathcal{V}(\mathfrak{g})$ is a birational morphism. We shall define a $G$-geometric crystal structure on $\mathcal{V}(\mathfrak{g})$ by using the Dynkin diagram automorphism $\sigma$ except for $A_{2 n}^{(2)}$. This $\sigma$ induces an automorphism of $W\left(\varpi_{1}\right)$, which is also denoted by $\sigma: W\left(\varpi_{1}\right) \longrightarrow W\left(\varpi_{1}\right)$. In the subsequent subsections, we shall show the following theorems by case-by-case arguments.

Theorem 5.1. (1) Case $\mathfrak{g} \neq A_{2 n}^{(2) \dagger}$. For $x=\left(x_{1}, \cdots, x_{k}\right) \in\left(\mathbb{C}^{\times}\right)^{k}$, there exist a unique $y=\left(y_{1}, \cdots, y_{k}\right) \in\left(\mathbb{C}^{\times}\right)^{k}$ and a positive rational function a $(x)$ such that

$$
v(y)=a(x) \sigma(v(x)), \quad \varepsilon_{\sigma(i)}(v(y))=\varepsilon_{i}(v(x)) \quad \text { if } i, \sigma(i) \neq 0 .
$$

(2) Case $\mathfrak{g}=A_{2 n}^{(2) \dagger}$. Associated with $w_{1}$ and $w_{2}$ as in the previous section, we define

$$
\begin{aligned}
\mathcal{V}(\mathfrak{g}) & :=\left\{v_{1}(x)=Y_{0}\left(x_{0}\right) Y_{1}\left(x_{1}\right) \cdots Y_{n}\left(x_{n}\right) Y_{n-1}\left(\bar{x}_{n-1}\right) \cdots Y_{1}\left(\bar{x}_{1}\right) v_{1} \mid x_{i}, \bar{x}_{i} \in \mathbb{C}^{\times}\right\}, \\
\mathcal{V}_{2}(\mathfrak{g}) & :=\left\{v_{2}(y)=Y_{n}\left(y_{n}\right) \cdots Y_{1}\left(y_{1}\right) Y_{0}\left(y_{0}\right) Y_{1}\left(\bar{y}_{1}\right) \cdots Y_{n-1}\left(\bar{y}_{n-1}\right) v_{\bar{n}} \mid y_{i}, \bar{y}_{i} \in \mathbb{C}^{\times}\right\} .
\end{aligned}
$$

For any $x \in\left(\mathbb{C}^{\times}\right)^{2 n}$ there exist a unique $y \in\left(\mathbb{C}^{\times}\right)^{2 n}$ and a rational function $a(x)$ such that $v_{2}(y)=a(x) v_{1}(x)$. 

$(5.4)$

Now, using this theorem, we define the rational mapping
$\bar{\sigma}: \mathcal{V}(\mathfrak{g}) \longrightarrow \mathcal{V}(\mathfrak{g})$
$\bar{\sigma}: \mathcal{V}(\mathfrak{g}) \longrightarrow \mathcal{V}_{2}(\mathfrak{g})$
$v(x) \quad \mapsto \quad v(y) \quad\left(\mathfrak{g} \neq A_{2 n}^{(2) \dagger}\right)$,
$v_{1}(x) \quad \mapsto \quad v_{2}(y) \quad\left(\mathfrak{g}=A_{2 n}^{(2) \dagger}\right)$.

Theorem 5.2. The rational mapping $\bar{\sigma}$ is birational. If we define

$$
\begin{cases}e_{0}^{c}:=\bar{\sigma}^{-1} \circ e_{\sigma(0)}^{c} \circ \bar{\sigma}, \quad \varepsilon_{0}:=\varepsilon_{\sigma(0)} \circ \bar{\sigma}, \quad \gamma_{0}:=\gamma_{\sigma(0)} \circ \bar{\sigma}, & \text { for } \mathfrak{g} \neq A_{2 n}^{(2) \dagger}, \\ e_{0}^{c}:=\bar{\sigma}^{-1} \circ e_{0}^{c} \circ \bar{\sigma}, \quad \varepsilon_{0}:=\varepsilon_{0} \circ \bar{\sigma}, \quad \gamma_{0}:=\gamma_{0} \circ \bar{\sigma}, & \text { for } \mathfrak{g}=A_{2 n}^{(2) \dagger},\end{cases}
$$

then $\left(\mathcal{V}(\mathfrak{g}),\left\{e_{i}\right\}_{i \in I},\left\{\gamma_{i}\right\}_{i \in I},\left\{\varepsilon_{i}\right\}_{i \in I}\right)$ is an affine $\mathfrak{g}$-geometric crystal.

Remark. In the case $\mathfrak{g}=A_{2 n}^{(2) \dagger}, \mathcal{V}_{2}(\mathfrak{g})$ has a $\mathfrak{g}_{I \backslash\{n\}}$-geometric crystal structure. Thus, $e_{0}, \gamma_{0}, \varepsilon_{0}$ are well defined on $\mathcal{V}_{2}(\mathfrak{g})$.

The following lemma is obvious and it shows Theorem 5.2 partially.

Lemma 5.3. Suppose that $\mathfrak{g} \neq A_{2 n}^{(2) \dagger}$. If there exists $\bar{\sigma}$ as above and

$$
e_{\sigma(i)}^{c}=\bar{\sigma} \circ e_{i}^{c} \circ \bar{\sigma}^{-1}, \quad \gamma_{i}=\gamma_{\sigma(i)} \circ \bar{\sigma}, \quad \varepsilon_{i}=\varepsilon_{\sigma(i)} \circ \bar{\sigma},
$$

for $i \neq \sigma^{-1}(0), 0$, then we obtain

(1)

$$
\begin{gathered}
e_{0}^{c_{1}} e_{i}^{c_{2}}=e_{i}^{c_{2}} e_{0}^{c_{1}} \quad \text { if } a_{0 i}=a_{i 0}=0, \\
e_{0}^{c_{1}} e_{i}^{c_{1} c_{2}} e_{0}^{c_{2}}=e_{i}^{c_{2}} e_{0}^{c_{1} c_{2}} e_{i}^{c_{2}} \quad \text { if } a_{0 i}=a_{i 0}=-1, \\
e_{0}^{c_{1}} e_{i}^{c_{1}^{2} c_{2}} e_{0}^{c_{1} c_{2}} e_{i}^{c_{2}}=e_{i}^{c_{2}} e_{0}^{c_{1} c_{2}} e_{i}^{c_{1}^{2} c_{2}} e_{0}^{c_{1}} \quad \text { if } a_{0 i}=-2, a_{i 0}=-1, \\
\text { (2) } \gamma_{0}\left(e_{i}^{c}(v(x))\right)=c^{a_{i 0}} \gamma_{0}(v(x)) \text { and } \gamma_{i}\left(e_{0}^{c}(v(x))\right)=c^{a_{0 i}} \gamma_{i}(v(x)), \\
(3) \varepsilon_{0}\left(e_{0}^{c}(v(x))\right)=c^{-1} \varepsilon_{0}(v(x)) .
\end{gathered}
$$

Proof. For example, we have

$$
\begin{aligned}
\gamma_{0}\left(e_{i}^{c}(v(x))\right) & =\gamma_{\sigma(0)}\left(\bar{\sigma} e_{i}^{c} \bar{\sigma}^{-1}(\bar{\sigma}(v(x)))\right) \\
& =\gamma_{\sigma(0)}\left(e_{\sigma(i)}^{c}(\bar{\sigma}(v(x)))\right)=c^{a_{\sigma(i), \sigma(0)}} \gamma_{\sigma(0)}(\bar{\sigma}(v(x))) \\
& =c^{a_{i, 0}} \gamma_{0}(v(x)),
\end{aligned}
$$

where we use $a_{\sigma(0), \sigma(i)}=a_{0 i}$ in the last equality. The other assertions are obtained similarly.

In the rest of this section, we shall prove Theorems 5.1 and 5.2 in each case.

5.3. $A_{n}^{(1)}$-case. We have $w_{1}:=s_{n} s_{n-1} \cdots s_{2} s_{1}$, and

$$
\mathcal{V}\left(A_{n}^{(1)}\right):=\left\{Y_{n}\left(x_{n}\right) \cdots Y_{2}\left(x_{2}\right) Y_{1}\left(x_{1}\right) v_{1} \mid x_{i} \in \mathbb{C}^{\times}\right\} \subset W\left(\varpi_{1}\right) .
$$

Since $\exp \left(c^{-1} f_{i}\right)=1+c^{-1} f_{i}$ on $W\left(\varpi_{1}\right), v(x)=Y_{n}\left(x_{n}\right) \cdots Y_{2}\left(x_{2}\right) Y_{1}\left(x_{1}\right) v_{1}$ is explicitly written as

$$
v(x)=v\left(x_{1}, \cdots, x_{n}\right)=\left(\sum_{i=1}^{n} x_{i} v_{i}\right)+v_{n+1} .
$$


Let $\sigma: \alpha_{k} \mapsto \alpha_{k+1}(k \in I)$ be the Dynkin diagram automorphism for $A_{n}^{(1)}$, which gives rise to the automorphism $\sigma: W\left(\varpi_{1}\right) \rightarrow W\left(\varpi_{1}\right)$. We have $\sigma v_{i}=v_{i+1}$. Then, we obtain

$$
\sigma(v(x))=v_{1}+\sum_{i=1}^{n} x_{i} v_{i+1} .
$$

Then the equation $v(y)=a(x) \sigma(v(x))$, i.e.

$$
\sum_{i=1}^{n} y_{i} v_{i}+v_{n+1}=a(x)\left(v_{1}+\sum_{i=1}^{n} x_{i} v_{i+1}\right)
$$

is solved by

$$
a(x)=\frac{1}{x_{n}}, \quad y_{1}=\frac{1}{x_{n}}, \quad y_{i}=\frac{x_{i-1}}{x_{n}}(i=2, \cdots, n)
$$

that is,

$$
\bar{\sigma}\left(v\left(x_{1} \cdots, x_{n}\right)\right)=v\left(\frac{1}{x_{n}}, \frac{x_{1}}{x_{n}}, \cdots, \frac{x_{n-1}}{x_{n}}\right) .
$$

The $A_{n}$-geometric crystal structure on $\mathcal{V}\left(A_{n}^{(1)}\right)$ induced from the one on $B_{w_{1}}^{-}$is given by:

$$
\begin{gathered}
\gamma_{1}(v(x))=\frac{x_{1}^{2}}{x_{2}}, \quad \gamma_{i}(v(x))=\frac{x_{i}^{2}}{x_{i-1} x_{i+1}} \quad(i=2, \cdots, n-1), \quad \gamma_{n}(v(x))=\frac{x_{n}^{2}}{x_{n-1}} \\
\varepsilon_{i}(v(x))=\frac{x_{i+1}}{x_{i}} \quad(i=1, \cdots, n-1), \quad \varepsilon_{n}(v(x))=\frac{1}{x_{n}} .
\end{gathered}
$$

Then we have

$$
\varepsilon_{i+1}(\bar{\sigma}(v(x)))= \begin{cases}\frac{x_{i+1}}{x_{i}} & \text { if } i=1, \cdots, n-2, \\ \frac{x_{n}}{x_{n-1}} & \text { if } i=n-1,\end{cases}
$$

which implies $\varepsilon_{\sigma(i)}(\bar{\sigma}(v(x)))=\varepsilon_{i}(v(x))$, and then we have completed the proof of Theorem 5.1 in this case.

Now, we define $e_{0}^{c}, \gamma_{0}$ and $\varepsilon_{0}$ by

$$
e_{0}^{c}:=\bar{\sigma}^{-1} \circ e_{1}^{c} \circ \bar{\sigma}, \quad \gamma_{0}:=\gamma_{1} \circ \bar{\sigma}, \quad \varepsilon_{0}:=\varepsilon_{1} \circ \bar{\sigma} .
$$

Their explicit forms are

$$
\begin{aligned}
& e_{0}^{c}(v(x))=v\left(\frac{x_{1}}{c}, \frac{x_{2}}{c}, \cdots, \frac{x_{n}}{c}\right), \\
& \gamma_{0}(v(x))=\frac{1}{x_{1} x_{n}}, \quad \varepsilon_{0}(v(x))=x_{1} .
\end{aligned}
$$

Thus, we can check (5.6) easily, and then Lemma 5.3 reduces the proof of Theorem 5.2 to the statements:

$$
\begin{aligned}
& e_{0}^{c_{1}} e_{n}^{c_{1} c_{2}} e_{0}^{c_{2}}=e_{n}^{c_{2}} e_{0}^{c_{1} c_{2}} e_{n}^{c_{1}}, \\
& \gamma_{0}\left(e_{n}^{c}(v(x))\right)=c^{-1} \gamma_{0}(v(x)), \quad \gamma_{n}\left(e_{0}^{c}(v(x))\right)=c^{-1} \gamma_{n}(v(x)) .
\end{aligned}
$$

These are immediate from (5.9)-(5.14). Thus, we obtain Theorem 5.2 , 
5.4. $B_{n}^{(1)}$-case. We have $w_{1}=s_{1} \cdots s_{n-1} s_{n} s_{n-1} \cdots s_{1}$, and

$$
\mathcal{V}\left(B_{n}^{(1)}\right):=\left\{v(x)=Y_{1}\left(x_{1}\right) \cdots Y_{n}\left(x_{n}\right) Y_{n-1}\left(\bar{x}_{n-1}\right) \cdots Y_{1}\left(\bar{x}_{1}\right) v_{1} \mid x_{i}, \bar{x}_{i} \in \mathbb{C}^{\times}\right\} .
$$

It follows from the explicit description of $W\left(\varpi_{1}\right)$ as in 4.3 that $y_{i}\left(c^{-1}\right)=\exp \left(c^{-1} f_{i}\right)$ on $W\left(\varpi_{1}\right)$ can be written as:

$$
\exp \left(c^{-1} f_{i}\right)= \begin{cases}1+c^{-1} f_{i} & i \neq n \\ 1+c^{-1} f_{n}+\frac{1}{2 c^{2}} f_{n}^{2} & i=n\end{cases}
$$

Therefore, we have

$$
\begin{aligned}
v\left(x_{1}, \cdots, x_{n}, \bar{x}_{n-1}, \cdots, \bar{x}_{1}\right) & =\left(\sum_{i=1}^{n} \xi_{i}(x) v_{i}\right)+x_{n} v_{0}+\left(\sum_{i=2}^{n} x_{i-1} v_{\bar{i}}\right)+v_{\overline{1}}, \\
\text { where } \xi_{i}(x): & = \begin{cases}\frac{x_{1} \bar{x}_{1} \bar{x}_{i-1}+x_{i} \bar{x}_{i}}{x_{i-1}} & i \neq 1, n, \\
\frac{x_{n-1} \bar{x}_{n-1}+x_{n}^{2}}{x_{n-1}} & i=n .\end{cases}
\end{aligned}
$$

Since $\sigma v_{1}=v_{\overline{1}}, \sigma v_{\overline{1}}=v_{1}$ and $\sigma v_{k}=v_{k}$ otherwise, we have

$$
\sigma(v(x))=v_{1}+\left(\sum_{i=2}^{n} \xi_{i}(x) v_{i}\right)+x_{n} v_{0}+\left(\sum_{i=2}^{n} x_{i-1} v_{\bar{i}}\right)+x_{1} \bar{x}_{1} v_{\overline{1}} .
$$

The equation $v(y)=a(x) \sigma(v(x))\left(x, y \in\left(\mathbb{C}^{\times}\right)^{2 n-1}\right)$ has a unique solution:

$$
\begin{aligned}
a(x)=\frac{1}{x_{1} \bar{x}_{1}}, \quad y_{i} & =a(x) x_{i}=\frac{x_{i}}{x_{1} \bar{x}_{1}} \quad(1 \leq i \leq n), \\
\bar{y}_{i} & =a(x) \bar{x}_{i}=\frac{\bar{x}_{i}}{x_{1} \bar{x}_{1}} \quad(1 \leq i<n) .
\end{aligned}
$$

Hence we have the rational mapping:

$$
\bar{\sigma}(v(x)):=v(y)=v\left(\frac{x_{1}}{x_{1} \bar{x}_{1}}, \frac{x_{2}}{x_{1} \bar{x}_{1}}, \cdots, \frac{x_{n}}{x_{1} \bar{x}_{1}}, \frac{\bar{x}_{n-1}}{x_{1} \bar{x}_{1}}, \cdots, \frac{\bar{x}_{1}}{x_{1} \bar{x}_{1}}\right)
$$

By the explicit form of $\bar{\sigma}$ in (5.18), we have $\bar{\sigma}^{2}=\mathrm{id}$, which means that the morphism $\bar{\sigma}$ is birational. In this case, the second condition in Theorem 5.1 is trivial since $\sigma(i)=i$ if $i, \sigma(i) \neq 0$. Thus, the proof of Theorem 5.1 in this case is completed.

Now, we set $e_{0}^{c}:=\bar{\sigma} \circ e_{1}^{c} \circ \bar{\sigma}, \gamma_{0}:=\gamma_{1} \circ \bar{\sigma}$ and $\varepsilon_{0}:=\varepsilon_{1} \circ \bar{\sigma}$. 
The explicit forms of the $e_{i}$ 's, $\varepsilon_{i}$ 's and $\gamma_{i}$ 's are:

$$
\begin{aligned}
& e_{0}^{c}: \quad x_{1} \mapsto \quad x_{1} \frac{c x_{1} \bar{x}_{1}+x_{2} \bar{x}_{2}}{c\left(x_{1} \bar{x}_{1}+x_{2} \bar{x}_{2}\right)}, \quad x_{i} \mapsto \frac{x_{i}}{c}(2 \leq i \leq n), \\
& \bar{x}_{1} \mapsto \quad \bar{x}_{1} \frac{x_{1} \bar{x}_{1}+x_{2} \bar{x}_{2}}{c x_{1} \bar{x}_{1}+x_{2} \bar{x}_{2}}, \quad \bar{x}_{i} \mapsto \frac{\bar{x}_{i}}{c}(2 \leq i \leq n-1), \\
& e_{i}^{c}: \quad x_{i} \mapsto \quad x_{i} \frac{c x_{i} \bar{x}_{i}+x_{i+1} \bar{x}_{i+1}}{x_{i} \bar{x}_{i}+x_{i+1} \bar{x}_{i+1}}, \quad \bar{x}_{i} \mapsto \bar{x}_{i} \frac{c\left(x_{i} \bar{x}_{i}+x_{i+1} \bar{x}_{i+1}\right)}{c x_{i} \bar{x}_{i}+x_{i+1} \bar{x}_{i+1}}, \\
& x_{j} \mapsto \quad x_{j}, \quad \bar{x}_{j} \mapsto \bar{x}_{j}(j \neq i) \quad(1 \leq i<n-1), \\
& e_{n-1}^{c}: \quad x_{n-1} \mapsto \quad x_{n-1} \frac{c x_{n-1} \bar{x}_{n-1}+x_{n}^{2}}{x_{n-1} \bar{x}_{n-1}+x_{n}^{2}}, \quad \bar{x}_{n-1} \mapsto \bar{x}_{n-1} \frac{c\left(x_{n-1} \bar{x}_{n-1}+x_{n}^{2}\right)}{c x_{n-1} \bar{x}_{n-1}+x_{n}^{2}} \\
& x_{j} \mapsto \quad x_{j}, \quad \bar{x}_{j} \mapsto \bar{x}_{j}(j \neq n-1), \\
& e_{n}^{c}: \quad x_{n} \mapsto \quad c x_{n}, \quad x_{j} \mapsto x_{j} \quad \bar{x}_{j} \mapsto \bar{x}_{j}(j \neq n), \\
& \varepsilon_{0}(v(x))=\frac{x_{1} \bar{x}_{1}+x_{2} \bar{x}_{2}}{x_{1}}, \quad \varepsilon_{1}(v(x))=\frac{1}{x_{1}}\left(1+\frac{x_{2} \bar{x}_{2}}{x_{1} \bar{x}_{1}}\right), \\
& \varepsilon_{i}(v(x))=\frac{x_{i-1}}{x_{i}}\left(1+\frac{x_{i+1} \bar{x}_{i+1}}{x_{i} \bar{x}_{i}}\right)(2 \leq i \leq n-2), \\
& \varepsilon_{n-1}(v(x))=\frac{x_{n-2}}{x_{n-1}}\left(1+\frac{x_{n}^{2}}{x_{n-1} \bar{x}_{n-1}}\right), \varepsilon_{n}(v(x))=\frac{x_{n-1}}{x_{n}}, \\
& \gamma_{0}(v(x))=\frac{1}{x_{2} \bar{x}_{2}}, \gamma_{1}(v(x))=\frac{\left(x_{1} \bar{x}_{1}\right)^{2}}{x_{2} \bar{x}_{2}} \\
& \gamma_{i}(v(x))=\frac{\left(x_{i} \bar{x}_{i}\right)^{2}}{x_{i-1} \bar{x}_{i-1} x_{i+1} \bar{x}_{i+1}}(2 \leq i \leq n-2), \\
& \gamma_{n-1}(v(x))=\frac{\left(x_{n-1} \bar{x}_{n-1}\right)^{2}}{x_{n-2} \bar{x}_{n-2} x_{n}^{2}}, \quad \gamma_{n}(v(x))=\frac{x_{n}^{2}}{x_{n-1} \bar{x}_{n-1}} .
\end{aligned}
$$

Since $\sigma(i)=i$ for $i \neq 0,1$, the condition (5.6) in Lemma 5.3 can be easily seen by (5.18) and by the explicit form of $e_{i}, \gamma_{i}$ and $\varepsilon_{i}(i \in I)$. Thus, in order to prove Theorem 5.2, it suffices to show that

$$
\begin{aligned}
& e_{0}^{c_{1}} e_{1}^{c_{2}}=e_{1}^{c_{2}} e_{0}^{c_{1}}, \\
& \gamma_{0}\left(e_{1}^{c}(v(x))\right)=\gamma_{0}(v(x)), \quad \gamma_{1}\left(e_{0}^{c}(v(x))\right)=\gamma_{1}(v(x)) .
\end{aligned}
$$

It follows from the explicit formula above that

$$
\begin{aligned}
e_{0}^{c_{1}} e_{1}^{c_{2}}(v(x)) & =e_{1}^{c_{2}} e_{0}^{c_{1}}(v(x)) \\
& =v\left(x_{1} \frac{c_{1} c_{2} x_{1} \bar{x}_{1}+x_{2} \bar{x}_{2}}{c_{1}\left(x_{1} \bar{x}_{1}+x_{2} \bar{x}_{2}\right)}, \frac{x_{2}}{c_{1}}, \cdots, \frac{\bar{x}_{2}}{c_{1}}, \bar{x}_{1} \frac{c_{2}\left(x_{1} \bar{x}_{1}+x_{2} \bar{x}_{2}\right)}{c_{1} c_{2} x_{1} \bar{x}_{1}+x_{2} \bar{x}_{2}}\right),
\end{aligned}
$$

which implies (5.19). We get (5.20) immediately from the formula above and we complete the proof of Theorem 5.2 for $B_{n}^{(1)}$. 
5.5. $C_{n}^{(1)}$-case. We have $w_{1}=s_{0} s_{1} \cdots s_{n} s_{n-1} \cdots s_{1}$ and

$\mathcal{V}\left(C_{n}^{(1)}\right):=\left\{v(x)=Y_{0}\left(x_{0}\right) Y_{1}\left(x_{1}\right) \cdots Y_{n}\left(x_{n}\right) Y_{n-1}\left(\bar{x}_{n-1}\right) \cdots Y_{1}\left(\bar{x}_{1}\right) v_{1} \mid x_{i}, \bar{x}_{i} \in \mathbb{C}^{\times}\right\}$.

Due to the explicit description of $W\left(\varpi_{1}\right)$ in $₫ 4.4$, we obtain $y_{i}\left(c^{-1}\right)=\exp \left(c^{-1} f_{i}\right)=$ $1+c^{-1} f_{i}$ on $W\left(\varpi_{1}\right)$. Hence we have

$$
\begin{aligned}
& v\left(x_{0}, x_{1}, \cdots, x_{n}, \bar{x}_{n-1}, \cdots, \bar{x}_{1}\right) \\
& \quad=\left(\sum_{i=1}^{n} \xi_{i} v_{i}\right)+\left(\sum_{i=1}^{n} x_{i-1} v_{\bar{i}}\right) \text { where } \xi_{i}:= \begin{cases}\frac{x_{0}+x_{1} \bar{x}_{1}}{x_{0}} & i=1, \\
\frac{x_{i-1} \bar{x}_{i-1}+x_{i} \bar{x}_{i}}{x_{i-1}} & i \neq 1, n, \\
\frac{x_{n-1} \bar{x}_{n-1}+x_{n}}{x_{n-1}} & i=n .\end{cases}
\end{aligned}
$$

The explicit forms of $\varepsilon_{i}(x)(1 \leq i \leq n)$ are:

$$
\begin{aligned}
& \varepsilon_{i}(v(x))=\frac{x_{i-1}}{x_{i}}\left(1+\frac{x_{i+1} \bar{x}_{i+1}}{x_{i} \bar{x}_{i}}\right)(1 \leq i \leq n-2), \\
& \varepsilon_{n-1}(v(x))=\frac{x_{n-2}}{x_{n-1}}\left(1+\frac{x_{n}}{x_{n-1} \bar{x}_{n-1}}\right), \varepsilon_{n}(v(x))=\frac{x_{n-1}^{2}}{x_{n}} .
\end{aligned}
$$

Since $\sigma v_{i+1}=v_{\overline{n-i}}$ and $\sigma v_{\overline{i+1}}=v_{n-i}(0 \leq i<n)$, we have

$$
\sigma(v(x))=\left(\sum_{i=1}^{n} x_{n-i} v_{i}\right)+\left(\sum_{i=1}^{n} \xi_{n-i+1} v_{\bar{i}}\right) .
$$

We obtain a unique solution of the equations $v(y)=a(x) \sigma(v(x)), \varepsilon_{n-1}(v(y))=$ $\varepsilon_{1}(v(x))\left(x, y \in\left(\mathbb{C}^{\times}\right)^{2 n}\right)$ :

$$
\begin{aligned}
& a(x)=\frac{\bar{x}_{n-1}}{x_{n}}+\frac{1}{x_{n-1}}, \\
& y_{0}=x_{n}\left(\frac{\bar{x}_{n-1}}{x_{n}}+\frac{1}{x_{n-1}}\right)^{2}, \\
& y_{i}=\left(\bar{x}_{n-i-1}+\frac{x_{n-i} \bar{x}_{n-i}}{x_{n-i-1}}\right)\left(\frac{\bar{x}_{n-1}}{x_{n}}+\frac{1}{x_{n-1}}\right) \quad(1 \leq i<n), \\
& y_{n-1}=\left(1+\frac{x_{1} \bar{x}_{1}}{x_{0}}\right)\left(\frac{\bar{x}_{n-1}}{x_{n}}+\frac{1}{x_{n-1}}\right), \\
& y_{n}=x_{0}\left(\frac{\bar{x}_{n-1}}{x_{n}}+\frac{1}{x_{n-1}}\right)^{2}, \\
& \bar{y}_{n-1}=\frac{\left(x_{n-1} \bar{x}_{n-1}+x_{n}\right) x_{0} x_{1} \bar{x}_{1}}{\left(x_{0}+x_{1} \bar{x}_{1}\right) x_{n-1} x_{n}}, \\
& \bar{y}_{i}=\frac{\left(x_{n-1} \bar{x}_{n-1}+x_{n}\right) x_{n-i-1} x_{n-i} \bar{x}_{n-i}}{\left(x_{n-i-1} \bar{x}_{n-i-1}+x_{n-i} \bar{x}_{n-i}\right) x_{n-1} x_{n}} \quad(1 \leq i<n-1) .
\end{aligned}
$$

Now we have the rational mapping $\bar{\sigma}: \mathcal{V}\left(C_{n}^{(1)}\right) \longrightarrow \mathcal{V}\left(C_{n}^{(1)}\right)$ defined by $v(x) \mapsto v(y)$. By the above explicit from of $y$, we have $\bar{\sigma}^{2}=\mathrm{id}$, which means that $\bar{\sigma}$ is birational. 
By direct calculations, we have $\varepsilon_{n-i}(v(y))=\varepsilon_{i}(v(x))(1 \leq i \leq n-1)$, and we complete the proof of Theorem 5.1 for $C_{n}^{(1)}$.

Let us define $e_{0}^{c}:=\bar{\sigma} \circ e_{n}^{c} \circ \bar{\sigma}\left(\bar{\sigma}^{2}=\mathrm{id}\right), \gamma_{0}:=\gamma_{n} \circ \bar{\sigma}$ and $\varepsilon_{0}:=\varepsilon_{n} \circ \bar{\sigma}$. The explicit forms of $e_{i}, \gamma_{i}$ and $\varepsilon_{0}$ are

$$
\begin{aligned}
& e_{0}^{c}: \quad x_{0} \mapsto \quad x_{0} \frac{\left(c x_{0}+x_{1} \bar{x}_{1}\right)^{2}}{c\left(x_{0}+x_{1} \bar{x}_{1}\right)^{2}}, \quad x_{i} \mapsto x_{i} \frac{c x_{0}+x_{1} \bar{x}_{1}}{c\left(x_{0}+x_{1} \bar{x}_{1}\right)}(1 \leq i \leq n-1), \\
& x_{n} \mapsto \quad x_{n} \frac{\left(c x_{0}+x_{1} \bar{x}_{1}\right)^{2}}{c^{2}\left(x_{0}+x_{1} \bar{x}_{1}\right)^{2}}, \quad \bar{x}_{i} \mapsto \bar{x}_{i} \frac{c x_{0}+x_{1} \bar{x}_{1}}{c\left(x_{0}+x_{1} \bar{x}_{1}\right)}(1 \leq i \leq n-1), \\
& e_{i}^{c}: \quad x_{i} \mapsto \quad x_{i} \frac{c x_{i} \bar{x}_{i}+x_{i+1} \bar{x}_{i+1}}{x_{i} \bar{x}_{i}+x_{i+1} \bar{x}_{i+1}}, \quad \bar{x}_{i} \mapsto \bar{x}_{i} \frac{c\left(x_{i} \bar{x}_{i}+x_{i+1} \bar{x}_{i+1}\right)}{c x_{i} \bar{x}_{i}+x_{i+1} \bar{x}_{i+1}}, \\
& x_{j} \mapsto \quad x_{j}, \quad \bar{x}_{j} \mapsto \bar{x}_{j} \quad(j \neq i), \quad(1 \leq i<n-1), \\
& e_{n-1}^{c}: \quad x_{n-1} \mapsto \quad x_{n-1} \frac{c x_{n-1} \bar{x}_{n-1}+x_{n}}{x_{n-1} \bar{x}_{n-1}+x_{n}}, \quad \bar{x}_{n-1} \mapsto \bar{x}_{n-1} \frac{c\left(x_{n-1} \bar{x}_{n-1}+x_{n}\right)}{c x_{n-1} \bar{x}_{n-1}+x_{n}}, \\
& x_{j} \mapsto \quad x_{j}, \quad \bar{x}_{j} \mapsto \bar{x}_{j} \quad(j \neq n-1), \\
& e_{n}^{c}: \quad x_{n} \mapsto \quad c x_{n}, \quad x_{j} \mapsto x_{j}, \quad \bar{x}_{j} \mapsto \bar{x}_{j} \quad(j \neq n), \\
& \gamma_{0}(v(x))=\frac{x_{0}^{2}}{\left(x_{1} \bar{x}_{1}\right)^{2}}, \quad \gamma_{1}(v(x))=\frac{\left(x_{1} \bar{x}_{1}\right)^{2}}{x_{0} x_{2} \bar{x}_{2}} \\
& \gamma_{i}=\frac{\left(x_{i} \bar{x}_{i}\right)^{2}}{x_{i-1} \bar{x}_{i-1} x_{i+1} \bar{x}_{i+1}} \quad(2 \leq i \leq n-2), \\
& \gamma_{n-1}(v(x))=\frac{\left(x_{n-1} \bar{x}_{n-1}\right)^{2}}{x_{n-2} \bar{x}_{n-2} x_{n}}, \quad \gamma_{n}(v(x))=\frac{x_{n}^{2}}{\left(x_{n-1} \bar{x}_{n-1}\right)^{2}}, \\
& \varepsilon_{0}(v(x))=\frac{1}{x_{0}}\left(1+\frac{x_{1} \bar{x}_{1}}{x_{0}}\right)^{2} .
\end{aligned}
$$

Let us check the condition (5.6) in Lemma 5.3 The following are useful for this purpose:

$$
\begin{aligned}
& y_{i} \bar{y}_{i}=a(x)^{2} x_{n-i} \bar{x}_{n-i}, \quad y_{0}=a(x)^{2} x_{n}, \\
& a(v(y))=a(\bar{\sigma}(v(x)))=\frac{1}{a(v(x))} .
\end{aligned}
$$

Using these we can easily check the two conditions $\gamma_{i}=\gamma_{\sigma(i)} \circ \bar{\sigma}$ and $\varepsilon_{i}=\varepsilon_{\sigma(i)} \circ \bar{\sigma}$. The condition $e_{\sigma(i)}^{c}=\bar{\sigma} \circ e_{i}^{c} \circ \bar{\sigma}^{-1}$ for $i=2, \cdots, n-2$ is also immediate from (5.21) and (5.22). Next let us see the case $i=1, n-1$. We have

$$
a\left(e_{n-1}^{c}(v(y))\right)=\frac{y_{n}+c y_{n-1} \bar{y}_{n-1}}{y_{n-1} y_{n}} \cdot \frac{x_{1} \bar{x}_{1}+x_{0}}{c x_{1} \bar{x}_{1}+x_{0}}=\frac{1}{a(v(x))} .
$$

Using this, we can get $e_{n-i}^{c}=\bar{\sigma} \circ e_{1}^{c} \circ \bar{\sigma}^{-1}$ and then $e_{1}^{c}=\bar{\sigma} \circ e_{n-1}^{c} \circ \bar{\sigma}^{-1}$ since $\bar{\sigma}^{2}=$ id. Now, it remains to show that

$$
e_{0}^{c_{1}} e_{n}^{c_{2}}=e_{n}^{c_{2}} e_{0}^{c_{1}}, \quad \gamma_{0}\left(e_{n}^{c}(v(x))\right)=\gamma_{0}(v(x)), \quad \gamma_{n}\left(e_{0}^{c}(v(x))\right)=\gamma_{n}(v(x)) .
$$


They easily follow from the explicit form of $e_{0}^{c}$. Thus, the proof of Theorem 5.2 in this case is completed.

5.6. $D_{n}^{(1)}$-case. We have $w_{1}=s_{1} s_{2} \cdots s_{n-1} s_{n} s_{n-2} s_{n-3} \cdots s_{2} s_{1}$, and

$$
\begin{aligned}
& \mathcal{V}\left(D_{n}^{(1)}\right):=\left\{v(x)=Y_{1}\left(x_{1}\right) \cdots Y_{n-1}\left(x_{n-1}\right) Y_{n}\left(x_{n}\right) Y_{n-2}\left(\bar{x}_{n-2}\right) \cdots Y_{1}\left(\bar{x}_{1}\right) v_{1} \mid\right. \\
& \left.x_{i}, \bar{x}_{i} \in \mathbb{C}^{\times}\right\} \text {. }
\end{aligned}
$$

It follows from the explicit form of $W\left(\varpi_{1}\right)$ in 4.5 that $y_{i}\left(c^{-1}\right)=\exp \left(c^{-1} f_{i}\right)=$ $1+c^{-1} f_{i}$ on $W\left(\varpi_{1}\right)$. Thus, we have

$$
\begin{aligned}
& v(x)=\left(\sum_{i=1}^{n-1} \xi_{i}(x) v_{i}\right)+x_{n} v_{n}+\left(\sum_{i=2}^{n} x_{i-1} v_{\bar{i}}\right)+v_{\overline{1}}, \\
& \text { where } \quad \xi_{i}(x):= \begin{cases}\frac{x_{1} \bar{x}_{1}}{\frac{x_{i-1} \bar{x}_{i-1}+x_{i} \bar{x}_{i}}{x_{i-1}}} & i=1, n-1, \\
\frac{x_{n-2} \bar{x}_{n-2}+x_{n-1} x_{n}}{x_{n-2}} & i=n-1 .\end{cases}
\end{aligned}
$$

Since $\sigma v_{1}=v_{\overline{1}}, \sigma v_{\overline{1}}=v_{1}$ and $\sigma v_{k}=v_{k}$ otherwise, we have

$$
\sigma(v(x))=v_{1}+\left(\sum_{i=2}^{n} \xi_{i}(x) v_{i}\right)+x_{n} v_{n}+\left(\sum_{i=1}^{n-1} x_{i-1} v_{\bar{i}}\right)+\xi_{1} v_{\overline{1}}
$$

Then the equation $v(y)=a(x) \sigma(v(x))\left(x, y \in\left(\mathbb{C}^{\times}\right)^{2 n-2}\right)$ has the following unique solution:

$$
\begin{aligned}
a(x)=\frac{1}{x_{1} \bar{x}_{1}}, \quad y_{i} & =a(x) x_{i}=\frac{x_{i}}{x_{1} \bar{x}_{1}} \quad(1 \leq i \leq n), \\
\bar{y}_{i} & =a(x) \bar{x}_{i}=\frac{\bar{x}_{i}}{x_{1} \bar{x}_{1}} \quad(1 \leq i \leq n-2) .
\end{aligned}
$$

We define the rational mapping $\bar{\sigma}: \mathcal{V}\left(D_{n}^{(1)}\right) \longrightarrow \mathcal{V}\left(D_{n}^{(1)}\right)$ by

$$
\bar{\sigma}(v(x))=v\left(\frac{x_{1}}{x_{1} \bar{x}_{1}}, \frac{x_{2}}{x_{1} \bar{x}_{1}}, \cdots, \frac{x_{n}}{x_{1} \bar{x}_{1}}, \frac{\bar{x}_{n-2}}{x_{1} \bar{x}_{1}}, \cdots, \frac{\bar{x}_{1}}{x_{1} \bar{x}_{1}}\right) .
$$

It is immediate from (5.24) that $\bar{\sigma}^{2}=\mathrm{id}$, which implies the birationality of the morphism $\bar{\sigma}$. In this case, the second condition in Theorem 5.1 is trivial since $\sigma(i)=i$ if $i, \sigma(i) \neq 0$. Thus, the proof of Theorem 5.1 for $D_{n}^{(1)}$ is completed. 
Now, we set $e_{0}^{c}:=\bar{\sigma} \circ e_{1}^{c} \circ \bar{\sigma}, \gamma_{0}:=\gamma_{1} \circ \bar{\sigma}$ and $\varepsilon_{0}:=\varepsilon_{1} \circ \bar{\sigma}$. The explicit forms of $e_{i}, \varepsilon_{i}$ and $\gamma_{i}$ are:

$$
\begin{aligned}
& e_{0}^{c}: \quad x_{1} \mapsto \quad x_{1} \frac{c x_{1} \bar{x}_{1}+x_{2} \bar{x}_{2}}{c\left(x_{1} \bar{x}_{1}+x_{2} \bar{x}_{2}\right)}, \quad x_{i} \mapsto \frac{x_{i}}{c}(2 \leq i \leq n), \\
& \bar{x}_{1} \mapsto \quad \bar{x}_{1} \frac{x_{1} \bar{x}_{1}+x_{2} \bar{x}_{2}}{c x_{1} \bar{x}_{1}+x_{2} \bar{x}_{2}}, \quad \bar{x}_{i} \mapsto \frac{\bar{x}_{i}}{c}(2 \leq i \leq n-2), \\
& e_{i}^{c}: \quad x_{i} \mapsto \quad x_{i} \frac{c x_{i} \bar{x}_{i}+x_{i+1} \bar{x}_{i+1}}{x_{i} \bar{x}_{i}+x_{i+1} \bar{x}_{i+1}}, \quad \bar{x}_{i} \mapsto \bar{x}_{i} \frac{c\left(x_{i} \bar{x}_{i}+x_{i+1} \bar{x}_{i+1}\right)}{c x_{i} \bar{x}_{i}+x_{i+1} \bar{x}_{i+1}}, \\
& x_{j} \mapsto \quad x_{j}, \quad \bar{x}_{j} \mapsto \bar{x}_{j} \quad(j \neq i), \quad(1 \leq i \leq n-3), \\
& e_{n-2}^{c}: \quad x_{n-2} \mapsto \quad x_{n-2} \frac{c x_{n-2} \bar{x}_{n-2}+x_{n-1} x_{n}}{x_{n-2} \bar{x}_{n-2}+x_{n-1} x_{n}}, \\
& \bar{x}_{n-2} \mapsto \quad \bar{x}_{n-2} \frac{c\left(x_{n-2} \bar{x}_{n-2}+x_{n-1} x_{n}\right)}{c x_{n-2} \bar{x}_{n-2}+x_{n-1} x_{n}}, \\
& x_{j} \mapsto \quad x_{j}, \quad \bar{x}_{j} \mapsto \bar{x}_{j} \quad(j \neq n-2), \\
& e_{n-1}^{c}: \quad x_{n-1} \mapsto c x_{n-1}, \quad x_{j} \mapsto x_{j}, \quad \bar{x}_{j} \mapsto \bar{x}_{j} \quad(j \neq n-1), \\
& e_{n}^{c}: \quad x_{n} \mapsto \quad c x_{n}, \quad x_{j} \mapsto x_{j}, \quad \bar{x}_{j} \mapsto \bar{x}_{j} \quad(j \neq n), \\
& \varepsilon_{0}(v(x))=\frac{x_{1} \bar{x}_{1}+x_{2} \bar{x}_{2}}{x_{1}}, \quad \varepsilon_{1}(v(x))=\frac{1}{x_{1}}\left(1+\frac{x_{2} \bar{x}_{2}}{x_{1} \bar{x}_{1}}\right), \\
& \varepsilon_{i}(v(x))=\frac{x_{i-1}}{x_{i}}\left(1+\frac{x_{i+1} \bar{x}_{i+1}}{x_{i} \bar{x}_{i}}\right) \quad(2 \leq i \leq n-3), \\
& \varepsilon_{n-2}(v(x))=\frac{x_{n-3}}{x_{n-2}}\left(1+\frac{x_{n-1} x_{n}}{x_{n-2} \bar{x}_{n-2}}\right), \quad \varepsilon_{n-1}(v(x))=\frac{x_{n-2}}{x_{n-1}}, \\
& \varepsilon_{n}(v(x))=\frac{x_{n-2}}{x_{n}}, \\
& \gamma_{0}(v(x))=\frac{1}{x_{2} \bar{x}_{2}}, \gamma_{1}(v(x))=\frac{\left(x_{1} \bar{x}_{1}\right)^{2}}{x_{2} \bar{x}_{2}} \\
& \gamma_{i}(v(x))=\frac{\left(x_{i} \bar{x}_{i}\right)^{2}}{x_{i-1} \bar{x}_{i-1} x_{i+1} \bar{x}_{i+1}}(2 \leq i \leq n-3), \\
& \gamma_{n-2}(v(x))=\frac{\left(x_{n-2} \bar{x}_{n-2}\right)^{2}}{x_{n-3} \bar{x}_{n-3} x_{n-1} x_{n}}, \quad \gamma_{n-1}(v(x))=\frac{x_{n-1}^{2}}{x_{n-2} \bar{x}_{n-2}}, \\
& \gamma_{n}(v(x))=\frac{x_{n}^{2}}{x_{n-2} \bar{x}_{n-2}} .
\end{aligned}
$$

By these formulas, we can show Theorem 5.2 for $D_{n}^{(1)}$ similarly to the one for $B_{n}^{(1)}$.

5.7. $A_{2 n-1}^{(2)}$-case. We have $w_{1}=s_{1} s_{2} \cdots s_{n} s_{n-1} \cdots s_{2} s_{1}$, and

$$
\mathcal{V}\left(A_{2 n-1}^{(2)}\right):=\left\{v(x):=Y_{1}\left(x_{1}\right) \cdots Y_{n}\left(x_{n}\right) Y_{n-1}\left(\bar{x}_{n-1}\right) \cdots Y_{1}\left(\bar{x}_{1}\right) v_{1} \mid x_{i}, \bar{x}_{i} \in \mathbb{C}^{\times}\right\} .
$$


In this case, $y_{i}\left(c^{-1}\right)=\exp \left(c^{-1} f_{i}\right)=1+c^{-1} f_{i}$ on $W\left(\varpi_{1}\right)$, and we have

$$
\begin{aligned}
& v\left(x_{1}, \cdots, x_{n}, \bar{x}_{n-1}, \cdots, \bar{x}_{1}\right)=\left(\sum_{i=1}^{n} \xi_{i} v_{i}\right)+\left(\sum_{i=2}^{n} x_{i-1} v_{\bar{i}}\right)+v_{\overline{1}}, \\
& \text { where } \quad \xi_{i}:= \begin{cases}x_{1} \bar{x}_{1} & i=1, \\
\frac{x_{i-1} \bar{x}_{i-1}+x_{i} \bar{x}_{i}}{x_{i-1}} & i \neq 1, n, \\
\frac{x_{n-1} \bar{x}_{n-1}+x_{n}}{x_{n-1}} & i=n .\end{cases}
\end{aligned}
$$

Since $\sigma v_{1}=v_{\overline{1}}, \sigma v_{\overline{1}}=v_{1}$ and $\sigma v_{k}=v_{k}$ otherwise, we have

$$
\sigma(v(x))=v_{1}+\left(\sum_{i=2}^{n} \xi_{i} v_{i}\right)+\left(\sum_{i=2}^{n} x_{i-1} v_{\bar{i}}\right)+x_{1} \bar{x}_{1} v_{\bar{\top}} .
$$

Solving $v(y)=a(x) \sigma(v(x))\left(x, y \in\left(\mathbb{C}^{\times}\right)^{2 n-1}\right)$, we obtain a unique solution:

$$
\begin{gathered}
a(x)=\frac{1}{x_{1} \bar{x}_{1}}, \quad y_{i}=a(x) x_{i}=\frac{x_{i}}{x_{1} \bar{x}_{1}}, \quad \bar{y}_{i}=a(x) \bar{x}_{i}=\frac{\bar{x}_{i}}{x_{1} \bar{x}_{1}} \quad(1 \leq i \leq n-1), \\
y_{n}=\frac{x_{n}}{\left(x_{1} \bar{x}_{1}\right)^{2}} .
\end{gathered}
$$

Then we have

$$
\bar{\sigma}(v(x))=v\left(\frac{x_{1}}{x_{1} \bar{x}_{1}}, \frac{x_{2}}{x_{1} \bar{x}_{1}}, \cdots, \frac{x_{n}}{\left(x_{1} \bar{x}_{1}\right)^{2}}, \frac{\bar{x}_{n-1}}{x_{1} \bar{x}_{1}}, \cdots, \frac{\bar{x}_{1}}{x_{1} \bar{x}_{1}}\right) .
$$

By the explicit form of $\bar{\sigma}$ in (5.26) , we have $\bar{\sigma}^{2}=$ id, which means that the morphism $\bar{\sigma}$ is birational. In this case, the second condition in Theorem 5.1 is trivial since $\sigma(i)=i$ if $i, \sigma(i) \neq 0$. Thus, the proof of Theorem 5.1 for $A_{2 n-1}^{(2)}$ is completed.

Now, we set $e_{0}^{c}:=\bar{\sigma} \circ e_{1}^{c} \circ \bar{\sigma}, \gamma_{0}:=\gamma_{1} \circ \bar{\sigma}$ and $\varepsilon_{0}:=\varepsilon_{1} \circ \bar{\sigma}$. The explicit forms of $e_{i}, \varepsilon_{i}$ and $\gamma_{i}$ are:

$$
\begin{aligned}
& e_{0}^{c}: \quad x_{1} \mapsto \quad x_{1} \frac{c x_{1} \bar{x}_{1}+x_{2} \bar{x}_{2}}{c\left(x_{1} \bar{x}_{1}+x_{2} \bar{x}_{2}\right)}, \quad x_{i} \mapsto \frac{x_{i}}{c}(2 \leq i \leq n-1), \quad x_{n} \mapsto \frac{x_{n}}{c^{2}}, \\
& \bar{x}_{1} \mapsto \quad \bar{x}_{1} \frac{x_{1} \bar{x}_{1}+x_{2} \bar{x}_{2}}{c x_{1} \bar{x}_{1}+x_{2} \bar{x}_{2}}, \quad \bar{x}_{i} \mapsto \frac{\bar{x}_{i}}{c}(2 \leq i \leq n-1), \\
& e_{i}^{c}: \quad x_{i} \mapsto \quad x_{i} \frac{c x_{i} \bar{x}_{i}+x_{i+1} \bar{x}_{i+1}}{x_{i} \bar{x}_{i}+x_{i+1} \bar{x}_{i+1}}, \quad \bar{x}_{i} \mapsto \bar{x}_{i} \frac{c\left(x_{i} \bar{x}_{i}+x_{i+1} \bar{x}_{i+1}\right)}{c x_{i} \bar{x}_{i}+x_{i+1} \bar{x}_{i+1}}, \\
& x_{j} \mapsto \quad x_{j}, \quad \bar{x}_{j} \mapsto \bar{x}_{j} \quad(j \neq i) \quad(1 \leq i<n-1), \\
& e_{n-1}^{c}: \quad x_{n-1} \mapsto \quad x_{n-1} \frac{c x_{n-1} \bar{x}_{n-1}+x_{n}}{x_{n-1} \bar{x}_{n-1}+x_{n}}, \quad \bar{x}_{n-1} \mapsto \bar{x}_{n-1} \frac{c\left(x_{n-1} \bar{x}_{n-1}+x_{n}\right)}{c x_{n-1} \bar{x}_{n-1}+x_{n}}, \\
& e_{n}^{c}: \quad x_{n} \mapsto \quad x_{j}, \quad \bar{x}_{j} \mapsto \bar{x}_{j} \quad(j \neq n-1), \quad(j \neq n) . \\
& \quad x_{n}, \quad x_{j} \mapsto x_{j}, \quad \bar{x}_{j} \mapsto \bar{x}_{j} \quad(j \neq n) \\
& \varepsilon_{0}(v(x))=\frac{x_{1} \bar{x}_{1}+x_{2} \bar{x}_{2}}{x_{1}}, \quad \varepsilon_{1}(v(x))=\frac{1}{x_{1}}\left(1+\frac{x_{2} \bar{x}_{2}}{x_{1} \bar{x}_{1}}\right), \\
& \varepsilon_{i}(v(x))=\frac{x_{i-1}}{x_{i}}\left(1+\frac{x_{i+1} \bar{x}_{i+1}}{x_{i} \bar{x}_{i}}\right) \quad(2 \leq i \leq n-2), \\
& \varepsilon_{n-1}(v(x))=\frac{x_{n-2}}{x_{n-1}}\left(1+\frac{x_{n}}{x_{n-1} \bar{x}_{n-1}}\right), \quad \varepsilon_{n}(v(x))=\frac{x_{n-1}^{2}}{x_{n}},
\end{aligned}
$$




$$
\begin{aligned}
& \gamma_{0}(v(x))=\frac{1}{x_{2} \bar{x}_{2}}, \quad \gamma_{1}(v(x))=\frac{\left(x_{1} \bar{x}_{1}\right)^{2}}{x_{2} \bar{x}_{2}}, \\
& \gamma_{i}=\frac{\left(x_{i} \bar{x}_{i}\right)^{2}}{x_{i-1} \bar{x}_{i-1} x_{i+1} \bar{x}_{i+1}} \quad(2 \leq i \leq n-2), \\
& \gamma_{n-1}(v(x))=\frac{\left(x_{n-1} \bar{x}_{n-1}\right)^{2}}{x_{n-2} \bar{x}_{n-2} x_{n}}, \quad \gamma_{n}(v(x))=\frac{x_{n}^{2}}{\left(x_{n-1} \bar{x}_{n-1}\right)^{2}} .
\end{aligned}
$$

We can show Theorem 5.2 for $A_{2 n-1}^{(2)}$ similarly to the one for $B_{n}^{(1)}$.

5.8. $D_{n+1}^{(2)}$-case. We have $w_{1}=s_{0} s_{1} \cdots s_{n} s_{n-1} \cdots s_{2} s_{1}$ and

$\mathcal{V}\left(D_{n+1}^{(2)}\right):=\left\{v(x):=Y_{0}\left(x_{0}\right) Y_{1}\left(x_{1}\right) \cdots Y_{n}\left(x_{n}\right) Y_{n-1}\left(\bar{x}_{n-1}\right) \cdots Y_{1}\left(\bar{x}_{1}\right) v_{1} \mid x_{i}, \bar{x}_{i} \in \mathbb{C}^{\times}\right\}$.

It follows from the explicit description of $W\left(\varpi_{1}\right)$ as in 4.7 that on $W\left(\varpi_{1}\right)$ :

$$
y_{i}\left(c^{-1}\right)=\exp \left(c^{-1} f_{i}\right)= \begin{cases}1+c^{-1} f_{i} & i \neq 0, n, \\ 1+c^{-1} f_{i}+\frac{1}{2 c^{2}} f_{i}^{2} & i=0, n .\end{cases}
$$

Then we have

$$
v(x)=\left(\sum_{i=1}^{n} \xi_{i}(x) v_{i}\right)+x_{n} v_{0}+x_{0} \phi+\left(\sum_{i=2}^{n} x_{i-1} v_{\bar{i}}\right)+x_{0}^{2} v_{\overline{1}}
$$

where

$$
\xi_{i}(x):= \begin{cases}\frac{x_{0}^{2}+x_{1} \bar{x}_{1}}{x_{0}^{2}} & i=1, \\ \frac{x_{i-1} x_{i-1}+x_{i} \bar{x}_{i}}{x_{i-1}} & i \neq 1, n, \\ \frac{x_{n-1} \bar{x}_{n-1}+x_{n}^{2}}{x_{n-1}} & i=n .\end{cases}
$$

Since $\sigma v_{i+1}=v \overline{n-i}$ and $\sigma v_{\overline{i+1}}=v_{n-i}(0 \leq i<n)$ and $\sigma: v_{0} \leftrightarrow \phi$, we also have

$$
\sigma(v(x))=\left(\sum_{i=1}^{n-1} x_{n-i} v_{i}\right)+x_{0}^{2} v_{n}+\left(\sum_{i=1}^{n} \xi_{n-i+1} v_{\bar{i}}\right)+x_{n} \phi+x_{0} v_{0} .
$$

Solving $v(y)=a(x) \sigma(v(x))\left(x, y \in\left(\mathbb{C}^{\times}\right)^{2 n}\right)$, we get a unique solution:

$$
\begin{aligned}
& a(x)=\frac{x_{n-1} \bar{x}_{n-1}+x_{n}^{2}}{x_{n-1} x_{n}^{2}}, \\
& y_{0}=\frac{x_{n-1} \bar{x}_{n-1}+x_{n}^{2}}{x_{n-1} x_{n}}, \\
& y_{i}=\frac{\left(x_{n-i-1} \bar{x}_{n-i-1}+x_{n-i} \bar{x}_{n-i}\right)\left(x_{n-1} \bar{x}_{n-1}+x_{n}^{2}\right)}{x_{n-i-1} x_{n-1} x_{n}^{2}} \quad(1 \leq i<n), \\
& y_{n-1}=\frac{\left(x_{0}^{2}+x_{1} \bar{x}_{1}\right)\left(x_{n-1} \bar{x}_{n-1}+x_{n}^{2}\right)}{x_{0}^{2} x_{n-1} x_{n}^{2}}, \\
& y_{n}=\frac{x_{0}\left(x_{n-1} \bar{x}_{n-1}+x_{n}^{2}\right)}{x_{n-1} x_{n}^{2}}, \\
& \bar{y}_{i}=\frac{\left(x_{n-1} \bar{x}_{n-1}+x_{n}^{2}\right) x_{n-i-1} x_{n-i} \bar{x}_{n-i}}{\left(x_{n-i-1} \bar{x}_{n-i-1}+x_{n-i} \bar{x}_{n-i}\right) x_{n-1} x_{n}^{2}} \quad(1 \leq i \leq n-2), \\
& \bar{y}_{n-1}=\frac{\left(x_{n-1} \bar{x}_{n-1}+x_{n}^{2}\right) x_{0}^{2} x_{1} \bar{x}_{1}}{\left(x_{0}^{2}+x_{1} \bar{x}_{1}\right) x_{n-1} x_{n}^{2}} .
\end{aligned}
$$


Then we have the rational mapping $\bar{\sigma}: \mathcal{V}\left(D_{n+1}^{(2)}\right) \longrightarrow \mathcal{V}\left(D_{n+1}^{(2)}\right)$ defined by $v(x) \mapsto$ $v(y)$. The explicit forms of $\varepsilon_{i}(1 \leq i \leq n)$ are as follows:

$$
\begin{gathered}
\varepsilon_{1}(v(x))=\frac{x_{0}^{2}}{x_{1}}\left(1+\frac{x_{2} \bar{x}_{2}}{x_{1} \bar{x}_{1}}\right), \quad \varepsilon_{n}(v(x))=\frac{x_{n-1}}{x_{n}}, \\
\varepsilon_{i}(v(x))=\frac{x_{i-1}}{x_{i}}\left(1+\frac{x_{i+1} \bar{x}_{i+1}}{x_{i} \bar{x}_{i}}\right)(2 \leq i \leq n-2), \\
\varepsilon_{n-1}(v(x))=\frac{x_{n-2}}{x_{n-1}}\left(1+\frac{x_{n}^{2}}{x_{n-1} \bar{x}_{n-1}}\right) .
\end{gathered}
$$

Then we get easily that $\varepsilon_{n-i}(v(y))=\varepsilon_{i}(v(x))(1 \leq i \leq n-1)$, which finishes the proof of Theorem 5.1 for $D_{n+1}^{(2)}$.

Let us define $e_{0}^{c}:=\bar{\sigma} \circ e_{n}^{c} \circ \bar{\sigma}\left(\bar{\sigma}^{2}=\mathrm{id}\right), \gamma_{0}:=\gamma_{n} \circ \bar{\sigma}$ and $\varepsilon_{0}:=\varepsilon_{n} \circ \bar{\sigma}$. The explicit forms of $e_{i}, \gamma_{i}$ and $\varepsilon_{0}$ are

$$
\begin{aligned}
& e_{0}^{c}: \quad x_{0} \mapsto \quad x_{0} \frac{c^{2} x_{0}^{2}+x_{1} \bar{x}_{1}}{c\left(x_{0}^{2}+x_{1} \bar{x}_{1}\right)}, \quad x_{i} \mapsto x_{i} \frac{c^{2} x_{0}^{2}+x_{1} \bar{x}_{1}}{c^{2}\left(x_{0}^{2}+x_{1} \bar{x}_{1}\right)}(1 \leq i \leq n), \\
& \bar{x}_{i} \mapsto \quad \bar{x}_{i} \frac{c^{2} x_{0}^{2}+x_{1} \bar{x}_{1}}{c^{2}\left(x_{0}^{2}+x_{1} \bar{x}_{1}\right)}(1 \leq i \leq n-1), \\
& e_{i}^{c}: \quad x_{i} \mapsto \quad x_{i} \frac{c x_{i} \bar{x}_{i}+x_{i+1} \bar{x}_{i+1}}{x_{i} \bar{x}_{i}+x_{i+1} \bar{x}_{i+1}}, \quad \bar{x}_{i} \mapsto \bar{x}_{i} \frac{c\left(x_{i} \bar{x}_{i}+x_{i+1} \bar{x}_{i+1}\right)}{c x_{i} \bar{x}_{i}+x_{i+1} \bar{x}_{i+1}}, \quad(1 \leq i<n-1), \\
& x_{j}\left.\mapsto \quad x_{j}, \quad \bar{x}_{j} \mapsto \bar{x}_{j} \quad(j \neq i), \quad x_{n-1} \bar{x}_{n-1}+x_{n}^{2}\right) \\
& e_{n}^{c}: \quad x_{n-1} \frac{c x_{n-1} \bar{x}_{n-1}+x_{n}^{2}}{x_{n-1} \bar{x}_{n-1}+x_{n}^{2}}, \quad \bar{x}_{n-1} \mapsto \bar{x}_{n-1} \frac{c\left(x_{n-1} \bar{x}_{n-1}+x_{n}^{2}\right.}{x_{j} \mapsto \bar{x}_{j} \quad(j \neq n-1),} \\
& x_{j} \mapsto \quad x_{j}, \quad \bar{x}_{n}, \quad x_{j} \mapsto x_{j}, \quad \bar{x}_{j} \mapsto \bar{x}_{j} \quad(j \neq n), \\
& \gamma_{0}(v(x))=\frac{x_{0}^{2}}{x_{1} \bar{x}_{1}}, \quad \gamma_{1}(v(x))=\frac{\left(x_{1} \bar{x}_{1}\right)^{2}}{x_{0}^{2} x_{2} \bar{x}_{2}}, \\
& \gamma_{i}(v(x))=\frac{\left(x_{i} \bar{x}_{i}\right)^{2}}{x_{i-1} \bar{x}_{i-1} x_{i+1} \bar{x}_{i+1}}(2 \leq i \leq n-2), \\
& \gamma_{n-1}(v(x))=\frac{\left(x_{n-1} \bar{x}_{n-1}\right)^{2}}{x_{n-2} \bar{x}_{n-2} x_{n}^{2}}, \quad \gamma_{n}(v(x))=\frac{x_{n}^{2}}{x_{n-1} \bar{x}_{n-1}}, \\
& \varepsilon_{0}(v(x))=\frac{x_{0}^{2}+x_{1} \bar{x}_{1}}{x_{0}^{3}} .
\end{aligned}
$$

Then similarly to the case $C_{n}^{(1)}$, we can show Theorem 5.2 for $D_{n+1}^{(2)}$.

5.9. $A_{2 n}^{(2) \dagger}$-case. As in the beginning of this section, we have $w_{1}=s_{0} s_{1} \cdots s_{n} s_{n-1}$ $\cdots s_{2} s_{1}$ and

$\mathcal{V}\left(A_{2 n}^{(2) \dagger}\right):=\left\{v_{1}(x)=Y_{0}\left(x_{0}\right) Y_{1}\left(x_{1}\right) \cdots Y_{n}\left(x_{n}\right) Y_{n-1}\left(\bar{x}_{n-1}\right) \cdots Y_{1}\left(\bar{x}_{1}\right) v_{1} \mid x_{i}, \bar{x}_{i} \in \mathbb{C}^{\times}\right\}$.

By the explicit description of $W\left(\varpi_{1}\right)$ as in 4.8 on $W\left(\varpi_{1}\right)$ we have:

$$
y_{i}\left(c^{-1}\right)=\exp \left(c^{-1} f_{i}\right)= \begin{cases}1+c^{-1} f_{i} & i \neq n \\ 1+c^{-1} f_{n}+\frac{1}{2 c^{2}} f_{n}^{2} & i=n\end{cases}
$$


Then we have

$$
\begin{aligned}
v_{1}(x)=\left(\sum_{i=1}^{n} \xi_{i}(x) v_{i}\right)+x_{n} v_{0}+\left(\sum_{i=1}^{n} x_{i-1} v_{\bar{i}}\right) \\
\text { where } \xi_{i}(x):= \begin{cases}\frac{\frac{x_{0}+x_{1} \bar{x}_{1}}{x_{0}}}{\frac{x_{i-1} \bar{x}_{i-1}+x_{i} \bar{x}_{i}}{x_{i-1}}} & i \neq 1, n, \\
\frac{x_{n-1} \bar{x}_{n-1}+x_{n}^{2}}{x_{n-1}} & i=n .\end{cases}
\end{aligned}
$$

Next, for $w_{2}=s_{n} s_{n-1} \cdots s_{1} s_{0} s_{1} \cdots s_{n-1}$ we set

$\mathcal{V}_{2}\left(A_{2 n}^{(2) \dagger}\right):=\left\{v_{2}(y)=Y_{n}\left(y_{n}\right) \cdots Y_{1}\left(y_{1}\right) Y_{0}\left(y_{0}\right) Y_{1}\left(\bar{y}_{1}\right) \cdots Y_{n-1}\left(\bar{y}_{n-1}\right) v_{\bar{n}} \mid y_{i}, \bar{y}_{i} \in \mathbb{C}^{\times}\right\}$.

Then we have

$$
\begin{array}{r}
v_{2}(y)=\left(\sum_{i=1}^{n-1} y_{i} v_{i}\right)+y_{n}^{2} v_{n}+y_{n} v_{0}+\left(\sum_{i=1}^{n} \eta_{i}(y) v_{\bar{i}}\right) \\
\text { where } \eta_{i}(y):= \begin{cases}\frac{y_{0}+y_{1} \bar{y}_{1}}{y_{1} \bar{y}_{i-1}+y_{i} \bar{y}_{i}} & i=1, n, \\
\frac{y_{i-1} y_{i}}{\frac{y_{n-1} \bar{y}_{n-1}+y_{n}^{2}}{y_{n}^{2}}} & i=n .\end{cases}
\end{array}
$$

For $x \in\left(\mathbb{C}^{\times}\right)^{2 n}$ there exist a unique $y=\left(y_{0}, \cdots, \bar{y}_{1}\right) \in\left(\mathbb{C}^{\times}\right)^{2 n}$ and $a(x)$ such that $v_{2}(y)=a(x) v_{1}(x)$. They are given by

$$
\begin{aligned}
& a(x)=\frac{x_{n-1} \bar{x}_{n-1}+x_{n}^{2}}{x_{n-1} x_{n}^{2}}, \\
& y_{0}=a(x)^{2} x_{0}=\frac{x_{0}\left(x_{n-1} \bar{x}_{n-1}+x_{n}^{2}\right)^{2}}{\left(x_{n-1} x_{n}^{2}\right)^{2}}, \\
& y_{1}=a(x) \xi_{1}(x)=\frac{\left(x_{n-1} \bar{x}_{n-1}+x_{n}^{2}\right)\left(x_{0}+x_{1} \bar{x}_{1}\right)}{x_{0} x_{n-1} x_{n}^{2}}, \quad(1<i<n), \\
& y_{i}=a(x) \xi_{i}(x)=\frac{\left(x_{i-1} \bar{x}_{i-1}+x_{i} \bar{x}_{i}\right)\left(x_{n-1} \bar{x}_{n-1}+x_{n}^{2}\right)}{x_{i-1} x_{n-1} x_{n}^{2}} \quad \\
& y_{n}=a(x) x_{n}=\frac{x_{n-1} \bar{x}_{n-1}+x_{n}^{2}}{x_{n-1} x_{n}}, \\
& \bar{y}_{1}=a(x) \frac{x_{0} x_{1} \bar{x}_{1}}{x_{0}+x_{1} \bar{x}_{1}}=\frac{\left(x_{n-1} \bar{x}_{n-1}+x_{n}^{2}\right) x_{0} x_{1} \bar{x}_{1}}{\left(x_{0}+x_{1} \bar{x}_{1}\right) x_{n-1} x_{n}^{2}}, \\
& \bar{y}_{i}=\frac{\left(x_{n-1} \bar{x}_{n-1}+x_{n}^{2}\right) x_{i-1} x_{i} \bar{x}_{i}}{\left(x_{i-1} \bar{x}_{i-1}+x_{i} \bar{x}_{i}\right) x_{n-1} x_{n}^{2}} \quad(1<i \leq n-1) .
\end{aligned}
$$


They define a rational mapping $\bar{\sigma}: \mathcal{V}\left(A_{2 n}^{(2) \dagger}\right) \longrightarrow \mathcal{V}_{2}\left(A_{2 n}^{(2) \dagger}\right)\left(v_{1}(x) \mapsto v_{2}(y)\right)$.

The inverse $\bar{\sigma}^{-1}: \mathcal{V}_{2}\left(A_{2 n}^{(2) \dagger}\right) \longrightarrow \mathcal{V}\left(A_{2 n}^{(2) \dagger}\right)\left(v_{2}(y) \mapsto v_{1}(x)\right)$ is given by

$$
\begin{aligned}
& a(y):=\frac{y_{0} y_{1}}{y_{0}+y_{1} \bar{y}_{1}}(=a(x)), \\
& x_{0}=a(y)^{-1} \frac{y_{0}+y_{1} \bar{y}_{1}}{y_{1}} \\
& x_{i}=a(y)^{-1} \frac{y_{i} \bar{y}_{i}+y_{i+1} \bar{y}_{i+1}}{y_{i+1}} \quad(1 \leq i \leq n-2), \\
& x_{n-1}=a(y)^{-1} \frac{y_{n-1} \bar{y}_{n-1}+y_{n}^{2}}{y_{n}^{2}} \\
& x_{n}=a(y)^{-1} y_{n}, \\
& \bar{x}_{i}=a(y)^{-1} \frac{y_{i} \bar{y}_{i} y_{i+1}}{y_{i} \bar{y}_{i}+y_{i+1} \bar{y}_{i+1}} \quad(1 \leq i \leq n-2), \\
& \bar{x}_{n-1}=a(y)^{-1} \frac{y_{n-1} \bar{y}_{n-1} y_{n}^{2}}{y_{n-1} \bar{y}_{n-1}+y_{n}^{2}}
\end{aligned}
$$

which means that the morphism $\bar{\sigma}: \mathcal{V}\left(A_{2 n}^{(2) \dagger}\right) \longrightarrow \mathcal{V}_{2}\left(A_{2 n}^{(2) \dagger}\right)$ is birational. Thus, we obtain Theorem $5.1(2)$.

The actions of $e_{i}(i=0,1, \cdots, n-1)$ on $v_{2}(y)$ are induced from the ones on $Y_{\mathbf{i}_{2}}(y)\left(\mathbf{i}_{\mathbf{2}}=(n, \cdots, 1,0,1, \cdots, n-1)\right)$ since $e_{i} v_{\bar{n}}=0$ for $i=0,1, \cdots, n-1$. We also get $\gamma_{i}\left(v_{2}(y)\right)$ and $\varepsilon_{i}\left(v_{2}(y)\right)$ from the ones for $Y_{\mathbf{i}_{2}}(y)$ where $v_{2}(y)=\bar{\sigma}\left(v_{1}(x)\right)$ :

$$
\begin{aligned}
& e_{0}^{c}: y_{0} \mapsto c y_{0}, \\
& e_{1}^{c}: y_{1} \mapsto y_{1} \frac{c y_{1} \bar{y}_{1}+y_{0}}{y_{1} \bar{y}_{1}+y_{0}}, \quad \bar{y}_{1} \mapsto \bar{y}_{1} \frac{c\left(y_{1} \bar{y}_{1}+y_{0}\right)}{c y_{1} \bar{y}_{1}+y_{0}}, \\
& e_{i}^{c}: y_{i} \mapsto y_{i} \frac{c y_{i} \bar{y}_{i}+y_{i-1} \bar{y}_{i-1}}{y_{i} \bar{y}_{i}+y_{i-1} \bar{y}_{i-1}}, \quad \bar{y}_{i} \mapsto \bar{y}_{i} \frac{c\left(y_{i} \bar{y}_{i}+y_{i-1} \bar{y}_{i-1}\right)}{c y_{i} \bar{y}_{i}+y_{i-1} \bar{y}_{i-1}} \quad(i=2, \cdots, n-1), \\
& \gamma_{0}\left(v_{2}(y)\right)=\frac{y_{0}^{2}}{\left(y_{1} \bar{y}_{1}\right)^{2}}, \quad \gamma_{1}\left(v_{2}(y)\right)=\frac{\left(y_{1} \bar{y}_{1}\right)^{2}}{y_{0} y_{2} \bar{y}_{2}}, \\
& \gamma_{i}\left(v_{2}(y)\right)=\frac{\left(y_{i} \bar{y}_{i}\right)^{2}}{y_{i-1} \bar{y}_{i-1} y_{i+1} \bar{y}_{i+1}}(i=2, \cdots, n-2), \gamma_{n-1}\left(v_{2}(y)\right)=\frac{\left(y_{n-1} \bar{y}_{n-1}\right)^{2}}{y_{n-2} \bar{y}_{n-2} y_{n}^{2}}, \\
& \varepsilon_{0}\left(v_{2}(y)\right)=\frac{y_{1}^{2}}{y_{0}}, \varepsilon_{1}\left(v_{2}(y)\right)=\frac{y_{2}}{y_{1}}\left(1+\frac{y_{0}}{y_{1} \bar{y}_{1}}\right), \\
& \varepsilon_{n-1}\left(v_{2}(y)\right)=\frac{y_{n}^{2}}{y_{n-1}}\left(1+\frac{y_{n-2} \bar{y}_{n-2}}{y_{n-1} \bar{y}_{n-1}}\right), \\
& \varepsilon_{i}\left(v_{2}(y)\right)=\frac{y_{i+1}}{y_{i}}\left(1+\frac{y_{i-1} \bar{y}_{i-1}}{y_{i} \bar{y}_{i}}\right) \quad(i=2 \cdots, n-1) .
\end{aligned}
$$

The explicit forms of $\varepsilon_{i}\left(v_{1}(x)\right)$ and $\gamma_{i}\left(v_{1}(x)\right)(1 \leq i \leq n)$ are also induced from the ones for $Y_{\mathbf{i}_{1}}(x):=Y_{0}\left(x_{0}\right) \cdots Y_{1}\left(\bar{x}_{1}\right)$, and we define $\varepsilon_{0}\left(v_{1}(x)\right):=\varepsilon_{0}\left(v_{2}(y)\right)$ and 


$$
\begin{gathered}
\gamma_{0}\left(v_{1}(x)\right):=\gamma_{0}\left(v_{2}(y)\right)\left(v_{2}(y):=\bar{\sigma}\left(v_{1}(x)\right)\right): \\
\varepsilon_{0}\left(v_{1}(x)\right)=\frac{1}{x_{0}}\left(1+\frac{x_{1} \bar{x}_{1}}{x_{0}}\right)^{2}, \\
\varepsilon_{i}\left(v_{1}(x)\right)=\frac{x_{i-1}}{x_{i}}\left(1+\frac{x_{i+1} \bar{x}_{i+1}}{x_{i} \bar{x}_{i}}\right) \quad(1 \leq i \leq n-2), \\
\varepsilon_{n-1}\left(v_{1}(x)\right)=\frac{x_{n-2}}{x_{n-1}}\left(1+\frac{x_{n}^{2}}{x_{n-1} \bar{x}_{n-1}}\right), \quad \varepsilon_{n}\left(v_{1}(x)\right)=\frac{x_{n-1}}{x_{n}}, \\
\gamma_{0}\left(v_{1}(x)\right)=\frac{x_{0}^{2}}{\left(x_{1} \bar{x}_{1}\right)^{2}}, \gamma_{1}\left(v_{1}(x)\right)=\frac{\left(x_{1} \bar{x}_{1}\right)^{2}}{x_{0} x_{2} \bar{x}_{2}}, \\
\gamma_{i}\left(v_{1}(x)\right)=\frac{\left(x_{i} \bar{x}_{i}\right)^{2}}{x_{i-1} \bar{x}_{i-1} x_{i+1} \bar{x}_{i+1}}(2 \leq i \leq n-2), \\
\gamma_{n-1}\left(v_{1}(x)\right)=\frac{\left(x_{n-1} \bar{x}_{n-1}\right)^{2}}{x_{n-2} \bar{x}_{n-2} x_{n}^{2}}, \quad \gamma_{n}\left(v_{1}(x)\right)=\frac{x_{n}^{2}}{x_{n-1} \bar{x}_{n-1}} .
\end{gathered}
$$

For $i=0$, we define $e_{0}^{c}\left(v_{1}(x)\right)=\bar{\sigma}^{-1} \circ e_{0}^{c} \circ \bar{\sigma}\left(v_{1}(x)\right)=\bar{\sigma}^{-1} \circ e_{0}^{c}\left(v_{2}(y)\right)$. Then we get

$$
\begin{aligned}
e_{0}^{c}: x_{0} & \mapsto x_{0} \frac{\left(c x_{0}+x_{1} \bar{x}_{1}\right)^{2}}{c\left(x_{0}+x_{1} \bar{x}_{1}\right)^{2}}, \quad x_{i} \mapsto x_{i} \frac{c x_{0}+x_{1} \bar{x}_{1}}{c\left(x_{0}+x_{1} \bar{x}_{1}\right)}(1 \leq i \leq n), \\
\bar{x}_{i} & \mapsto \bar{x}_{i} \frac{c x_{0}+x_{1} \bar{x}_{1}}{c\left(x_{0}+x_{1} \bar{x}_{1}\right)}(1 \leq i \leq n-1), \\
e_{i}^{c}: x_{i} & \mapsto x_{i} \frac{c x_{i} \bar{x}_{i}+x_{i+1} \bar{x}_{i+1}}{x_{i} \bar{x}_{i}+x_{i+1} \bar{x}_{i+1}}, \quad \bar{x}_{i} \mapsto \bar{x}_{i} \frac{c\left(x_{i} \bar{x}_{i}+x_{i+1} \bar{x}_{i+1}\right)}{c x_{i} \bar{x}_{i}+x_{i+1} \bar{x}_{i+1}}(1 \leq i<n-1), \\
e_{n-1}^{c}: x_{n-1} & \mapsto x_{n-1} \frac{c x_{n-1} \bar{x}_{n-1}+x_{n}^{2}}{x_{n-1} \bar{x}_{n-1}+x_{n}^{2}}, \quad \bar{x}_{n-1} \mapsto \bar{x}_{n-1} \frac{c\left(x_{n-1} \bar{x}_{n-1}+x_{n}^{2}\right)}{c x_{n-1} \bar{x}_{n-1}+x_{n}^{2}}, \\
e_{n}^{c}: x_{n} & \mapsto c x_{n},
\end{aligned}
$$

where we omit the unchanged parts. In order to prove Theorem 5.2, it suffices to show the following:

$$
\begin{aligned}
& e_{i}^{c}=\bar{\sigma}^{-1} \circ e_{i}^{c} \circ \bar{\sigma}, \gamma_{i}=\gamma_{i} \circ \bar{\sigma}, \varepsilon_{i}=\varepsilon_{i} \circ \bar{\sigma}(i \neq 0, n), \\
& e_{0}^{c_{1}} e_{n}^{c_{2}}=e_{n}^{c_{2}} e_{0}^{c_{1}}, \\
& \gamma_{n}\left(e_{0}^{c}\left(v_{1}(x)\right)\right)=\gamma_{n}\left(v_{1}(x)\right), \quad \gamma_{0}\left(e_{n}^{c}\left(v_{1}(x)\right)\right)=\gamma_{0}\left(v_{1}(x)\right), \\
& \varepsilon_{0}\left(e_{0}^{c}\left(v_{1}(x)\right)\right)=c^{-1} \varepsilon_{0}\left(v_{1}(x)\right),
\end{aligned}
$$

which are immediate from the above formulae. Let us show (5.27). Set $v_{2}(y):=$ $\bar{\sigma}\left(v_{1}(x)\right)$ and $v_{1}\left(x^{\prime}\right):=\bar{\sigma}^{-1}\left(e_{i}^{c}\left(v_{2}(y)\right)\right)$ for $i=2, \cdots, n-2$. Then $x_{j}^{\prime}=x_{j}$ and $\bar{x}_{j}^{\prime}=\bar{x}_{j}$ for $j \neq i-1, i$, and we have

$$
\begin{aligned}
& a\left(v_{2}(y)\right)=a\left(v_{1}(x)\right), \\
& x_{i}^{\prime}=\frac{1}{a\left(v_{2}(y)\right)}\left(\bar{y}_{i+1}+\frac{c y_{i} \bar{y}_{i}}{y_{i+1}}\right)=x_{i} \frac{c x_{i} \bar{x}_{i}+x_{i+1} \bar{x}_{i+1}}{x_{i} \bar{x}_{i}+x_{i+1} \bar{x}_{i+1}}, \\
& \bar{x}_{i}^{\prime}=\frac{1}{a\left(v_{2}(y)\right)} \frac{c y_{i} \bar{y}_{i} y_{i+1}}{c y_{i} \bar{y}_{i}+y_{i+1} \bar{y}_{i+1}}=\bar{x}_{i} \frac{c\left(x_{i} \bar{x}_{i}+x_{i+1} \bar{x}_{i+1}\right)}{c x_{i} \bar{x}_{i}+x_{i+1} \bar{x}_{i+1}}, \\
& x_{i-1}^{\prime}=\frac{1}{a\left(v_{2}(y)\right)}\left(\bar{y}_{i}+\frac{c y_{i-1} \bar{y}_{i-1}}{y_{i}}\right)=x_{i-1}, \\
& \bar{x}_{i-1}^{\prime}=\frac{1}{a\left(v_{2}(y)\right)} \frac{c y_{i-1} \bar{y}_{i-1} y_{i}}{c y_{i} \bar{y}_{i}+y_{i-1} \bar{y}_{i-1}}=\bar{x}_{i-1},
\end{aligned}
$$


where the formula $y_{i} \bar{y}_{i}=a\left(v_{1}(x)\right)^{2} x_{i} \bar{x}_{i}$ is useful to obtain these results. Therefore we have $e_{i}^{c}=\bar{\sigma}^{-1} \circ e_{i}^{c} \circ \bar{\sigma}$ for $i=2, \cdots, n-2$. The others are obtained similarly.

\section{Ultra-DisCRETIZATION OF AFFINE GEOMETRIC CRYSTALS}

In this section, we shall see that the limit $B_{\infty}$ of perfect crystals as in 93 is isomorphic to the ultra-discretization of the geometric crystal obtained in the previous section.

Let $\mathcal{V}(\mathfrak{g})$ be the affine geometric crystal for $\mathfrak{g}$ as in the previous section. Let $m=\operatorname{dim} \mathcal{V}(\mathfrak{g})$. Then due to their explicit forms in the previous section, we have the following simple positive structure on $\mathcal{V}(\mathfrak{g})$ :

$$
\begin{aligned}
\Theta:\left(\mathbb{C}^{\times}\right)^{m} & \longrightarrow \mathcal{V}(\mathfrak{g}), \\
x & \mapsto v(x) .
\end{aligned}
$$

Therefore, we obtain an affine $\operatorname{crystal} \mathcal{U} D(\mathcal{V}(\mathfrak{g}))=\left(\mathcal{B}(\mathfrak{g}),\left\{\tilde{e}_{i}\right\}_{i \in I},\left\{\mathrm{wt}_{i}\right\}_{i \in I},\left\{\varepsilon_{i}\right\}_{i \in I}\right)$.

Theorem 6.1. For $\mathfrak{g}=A_{n}^{(1)}, B_{n}^{(1)}, C_{n}^{(1)}, D_{n}^{(1)}, A_{2 n-1}^{(2)}, D_{n+1}^{(2)}$ and $A_{2 n}^{(2) \dagger}$, let us denote its Langlands dual by $\mathfrak{g}^{L}$. Then we have the following isomorphism of crystals:

$$
\mathcal{U} D(\mathcal{V}(\mathfrak{g})) \cong B_{\infty}\left(\mathfrak{g}^{L}\right)
$$

Here $L: A_{n}^{(1)} \leftrightarrow A_{n}^{(1)}, L: B_{n}^{(1)} \leftrightarrow A_{2 n-1}^{(2)}, L: C_{n}^{(1)} \leftrightarrow D_{n+1}^{(2)}, L: D_{n}^{(1)} \leftrightarrow D_{n}^{(1)}$ and $L: A_{2 n}^{(2)} \leftrightarrow A_{2 n}^{(2) \dagger}$.

In the following subsections, we shall show Theorem 6.1 in each case.

The following formula is useful for this purpose:

$$
\mathcal{U} D\left(\frac{c x+y}{x+y}\right)=\max (c+x, y)-\max (x, y) .
$$

Note that, if $c=1$,

$$
\max (c+x, y)-\max (x, y)= \begin{cases}1 & x \geq y \\ 0 & x<y\end{cases}
$$

6.1. $A_{n}^{(1)}(n \geq 2)$. The crystal structure of $\mathcal{U} D\left(\mathcal{V}\left(A_{n}^{(1)}\right)\right)$ is given as follows: $\mathcal{B}\left(A_{n}^{(1)}\right)$ $=\mathbb{Z}^{n}$ and for $x=\left(x_{1}, \cdots, x_{n}\right) \in \mathbb{Z}^{n}$, we have

$$
\begin{aligned}
& \tilde{e}_{0}(x)=\left(x_{1}-1, x_{2}-1, \cdots, x_{n}-1\right), \\
& \tilde{e}_{i}(x)=\left(\cdots, x_{i}+1, \cdots\right) \quad(1 \leq i \leq n), \\
& \operatorname{wt}_{0}(x)=-x_{1}-x_{n}, \quad \operatorname{wt}_{1}(x)=2 x_{1}-x_{2}, \\
& \operatorname{wt}_{i}(x)=-x_{i-1}+2 x_{i}-x_{i+1} \quad(i=2, \cdots, n-1), \quad \operatorname{wt}_{n}(x)=2 x_{n}-x_{n-1}, \\
& \varepsilon_{0}(x)=x_{1}, \quad \varepsilon_{i}(x)=x_{i+1}-x_{i} \quad(i=1, \cdots, n-1), \quad \varepsilon_{n}(x)=-x_{n} .
\end{aligned}
$$

Comparing with the result in 3.4 and the above formulae, we easily see that the following map gives an isomorphism of $A_{n}^{(1)}$-crystals:

$$
\begin{aligned}
\mu: \mathcal{B}\left(A_{n}^{(1)}\right)=\mathbb{Z}^{n} & \longrightarrow B_{\infty}\left(A_{n}^{(1)}\right), \\
\left(x_{1}, \cdots, x_{n}\right) & \mapsto\left(b_{1}, \cdots, b_{n}, b_{n+1}\right)=\left(x_{1}, x_{2}-x_{1}, \cdots, x_{n}-x_{n-1},-x_{n}\right) .
\end{aligned}
$$

Thus we have proved Theorem 6.1 for $A_{n}^{(1)}$. 
6.2. $B_{n}^{(1)}(n \geq 3)$. Let us see the crystal structure of $\mathcal{U} D\left(\mathcal{V}\left(B_{n}^{(1)}\right)\right)=\left(\mathcal{B}\left(B_{n}^{(1)}\right),\left\{\tilde{e}_{i}\right\}\right.$, $\left.\left\{\mathrm{wt}_{i}\right\},\left\{\varepsilon_{i}\right\}\right)$. Due to the formula in 5.4 , we have

$$
\mathcal{B}\left(B_{n}^{(1)}\right)=\mathbb{Z}^{2 n-1}
$$

and for

$$
x=\left(x_{1}, \cdots, x_{n}, \bar{x}_{n-1}, \cdots, \bar{x}_{1}\right) \in \mathcal{B}\left(B_{n}^{(1)}\right),
$$

we have

$$
\begin{aligned}
& \tilde{e}_{0}(x)= \begin{cases}\left(x_{1}, x_{2}-1, \cdots, x_{n}-1, \cdots, \bar{x}_{2}-1, \bar{x}_{1}-1\right) & \text { if } x_{1}+\bar{x}_{1} \geq x_{2}+\bar{x}_{2}, \\
\left(x_{1}-1, x_{2}-1, \cdots, x_{n}-1, \cdots, \bar{x}_{2}-1, \bar{x}_{1}\right) & \text { if } x_{1}+\bar{x}_{1}<x_{2}+\bar{x}_{2},\end{cases} \\
& \tilde{e}_{i}(x)= \begin{cases}\left(x_{1}, \cdots, x_{i}+1, \cdots, \bar{x}_{i}, \cdots, \bar{x}_{1}\right) & \text { if } x_{i}+\bar{x}_{i} \geq x_{i+1}+\bar{x}_{i+1}, \\
\left(x_{1}, \cdots, x_{i}, \cdots, \bar{x}_{i}+1, \cdots, \bar{x}_{1}\right) & \text { if } x_{i}+\bar{x}_{i}<x_{i+1}+\bar{x}_{i+1},\end{cases} \\
& (i=1, \cdots, n-2) \text {, } \\
& \tilde{e}_{n-1}(x)= \begin{cases}\left(x_{1}, \cdots, x_{n-1}+1, x_{n}, \bar{x}_{n-1}, \cdots, \bar{x}_{1}\right) & \text { if } x_{n-1}+\bar{x}_{n-1} \geq 2 x_{n}, \\
\left(x_{1}, \cdots, x_{n-1}, x_{n}, \bar{x}_{n-1}+1, \cdots, \bar{x}_{1}\right) & \text { if } x_{n-1}+\bar{x}_{n-1}<2 x_{n},\end{cases} \\
& \tilde{e}_{n}(x)=\left(x_{1}, \cdots, x_{n-1}, x_{n}+1, \bar{x}_{n-1}, \cdots, \bar{x}_{1}\right) \text {, } \\
& \mathrm{wt}_{0}(x)=-\left(x_{2}+\bar{x}_{2}\right), \quad \mathrm{wt}_{1}(x)=2\left(x_{1}+\bar{x}_{1}\right)-\left(x_{2}+\bar{x}_{2}\right), \\
& \mathrm{wt}_{i}(x)=-\left(x_{i-1}+\bar{x}_{i-1}\right)+2\left(x_{i}+\bar{x}_{i}\right)-\left(x_{i+1}+\bar{x}_{i+1}\right) \quad(i=2, \cdots, n-2) \text {, } \\
& \mathrm{wt}_{n-1}(x)=-\left(x_{n-2}+\bar{x}_{n-2}\right)+2\left(x_{n-1}+\bar{x}_{n-1}\right)-2 x_{n} \text {, } \\
& \mathrm{wt}_{n}(x)=2 x_{n}-\left(x_{n-1}+\bar{x}_{n-1}\right) \text {, } \\
& \varepsilon_{0}(x)=\max \left(x_{1}+\bar{x}_{1}, x_{2}+\bar{x}_{2}\right)-x_{1}=\bar{x}_{1}+\left(x_{2}+\bar{x}_{2}-x_{1}-\bar{x}_{1}\right)_{+}, \\
& \varepsilon_{1}(x)=\left(x_{2}+\bar{x}_{2}-x_{1}-\bar{x}_{1}\right)_{+}-x_{1}, \\
& \varepsilon_{i}(x)=x_{i-1}-x_{i}+\left(x_{i+1}+\bar{x}_{i+1}-x_{i}-\bar{x}_{i}\right)_{+}(i=2, \cdots, n-2), \\
& \varepsilon_{n-1}(x)=x_{n-2}-x_{n-1}+\left(2 x_{n}-x_{n-1}-\bar{x}_{n-1}\right)_{+}, \quad \varepsilon_{n}(x)=x_{n-1}-x_{n} .
\end{aligned}
$$

Comparing the result in $\$ 3.8$ and the above formulae, it is easy to see that the following map gives an isomorphism of $A_{2 n-1}^{(2)}$-crystals:

$$
\begin{aligned}
\mu: \mathcal{B}\left(B_{n}^{(1)}\right) & \longrightarrow B_{\infty}\left(A_{2 n-1}^{(2)}\right) \\
\left(x_{1}, \cdots, x_{n}, \cdots, \bar{x}_{1}\right) & \mapsto \quad\left(b_{1}, \cdots, b_{n}, \bar{b}_{n}, \cdots, \bar{b}_{1}\right),
\end{aligned}
$$

where

$$
\begin{aligned}
& b_{1}=\bar{x}_{1}, \quad b_{i}=\bar{x}_{i}-\bar{x}_{i-1}(i=2, \cdots, n-1), \quad b_{n}=x_{n}-\bar{x}_{n-1}, \\
& \bar{b}_{i}=x_{i-1}-x_{i}(i=2, \cdots, n), \bar{b}_{1}=-x_{1} .
\end{aligned}
$$

We have proved Theorem 6.1 for $B_{n}^{(1)}$. 
6.3. $C_{n}^{(1)}(n \geq 2)$. Let us see the crystal structure of $\mathcal{U} D\left(\mathcal{V}\left(C_{n}^{(1)}\right)\right)=\left(\mathcal{B}\left(C_{n}^{(1)}\right),\left\{\tilde{e}_{i}\right\}\right.$, $\left.\left\{\mathrm{wt}_{i}\right\},\left\{\varepsilon_{i}\right\}\right)$. We have $\mathcal{B}\left(C_{n}^{(1)}\right)=\mathbb{Z}^{2 n}$ and for $x=\left(x_{0}, x_{1}, \cdots, x_{n}, \bar{x}_{n-1}, \cdots, \bar{x}_{1}\right) \in$ $\mathcal{B}\left(C_{n}^{(1)}\right)$, we have

$$
\begin{aligned}
& \tilde{e}_{0}(x)=\left\{\begin{array}{cc}
\left(x_{0}+1, x_{1}, \cdots, x_{n}, \bar{x}_{n-1}, \cdots, \bar{x}_{2}, \bar{x}_{1}\right) & \text { if } x_{0} \geq x_{1}+\bar{x}_{1}, \\
\left(x_{0}-1, \cdots, x_{n-1}-1, x_{n}-2, \bar{x}_{n-1}-1, \cdots, \bar{x}_{2}-1, \bar{x}_{1}-1\right) & \text { if } x_{0}<x_{1}+\bar{x}_{1},
\end{array}\right. \\
& \tilde{e}_{i}(x)=\left\{\begin{array}{rr}
\left(x_{0}, \cdots, x_{i}+1, \cdots, \bar{x}_{i}, \cdots, \bar{x}_{1}\right) & \text { if } x_{i}+\bar{x}_{i} \geq x_{i+1}+\bar{x}_{i+1}, \\
\left(x_{0}, \cdots, x_{i}, \cdots, \bar{x}_{i}+1, \cdots, \bar{x}_{1}\right) & \text { if } x_{i}+\bar{x}_{i}<x_{i+1}+\bar{x}_{i+1},
\end{array}\right. \\
& \tilde{e}_{n-1}(x)=\left\{\begin{array}{rr}
(i=1, \cdots, n-2), \\
\left(x_{0}, \cdots, x_{n-1}+1, x_{n}, \bar{x}_{n-1}, \cdots, \bar{x}_{1}\right) & \text { if } x_{n-1}+\bar{x}_{n-1} \geq x_{n},
\end{array}\right.
\end{aligned}
$$

$$
\begin{aligned}
& \mathrm{wt}_{0}(x)=2 x_{0}-2\left(x_{1}+\bar{x}_{1}\right), \quad \mathrm{wt}_{1}(x)=2\left(x_{1}+\bar{x}_{1}\right)-\left(x_{0}+x_{2}+\bar{x}_{2}\right), \\
& \mathrm{wt}_{i}(x)=-\left(x_{i-1}+\bar{x}_{i-1}\right)+2\left(x_{i}+\bar{x}_{i}\right)-\left(x_{i+1}+\bar{x}_{i+1}\right)(i=2, \cdots, n-2) \\
& \mathrm{wt}_{n-1}(x)=-\left(x_{n-2}+\bar{x}_{n-2}\right)+2\left(x_{n-1}+\bar{x}_{n-1}\right)-x_{n}, \\
& \mathrm{wt}_{n}(x)=2 x_{n}-2\left(x_{n-1}+\bar{x}_{n-1}\right), \\
& \varepsilon_{0}(x)=-x_{0}+2\left(x_{1}+\bar{x}_{1}-x_{0}\right)_{+}, \\
& \varepsilon_{i}(x)=x_{i-1}-x_{i}+\left(x_{i+1}+\bar{x}_{i+1}-x_{i}-\bar{x}_{i}\right)_{+}(i=1, \cdots, n-2), \\
& \varepsilon_{n-1}(x)=x_{n-2}-x_{n-1}+\left(x_{n}-x_{n-1}-\bar{x}_{n-1}\right)_{+}, \quad \varepsilon_{n}(x)=2 x_{n-1}-x_{n} .
\end{aligned}
$$

Comparing the result in 3.9 and the above formulae, we see that the following map gives an isomorphism of $D_{n+1}^{(2)}$-crystals:

$$
\begin{aligned}
\mu: \mathcal{B}\left(C_{n}^{(1)}\right) & \longrightarrow B_{\infty}\left(D_{n+1}^{(2)}\right) \\
\left(x_{0}, x_{1}, \cdots, x_{n}, \cdots, \bar{x}_{1}\right) & \mapsto\left(b_{1}, \cdots, b_{n}, \bar{b}_{n}, \cdots, \bar{b}_{1}\right),
\end{aligned}
$$

where

$$
\begin{aligned}
& b_{1}=\bar{x}_{1}, \quad b_{i}=\bar{x}_{i}-\bar{x}_{i-1}(i=2, \cdots, n-1), \quad b_{n}=\frac{1}{2} x_{n}-\bar{x}_{n-1}, \\
& \bar{b}_{n}=x_{n-1}-\frac{1}{2} x_{n}, \quad \bar{b}_{i}=x_{i-1}-x_{i}(i=1, \cdots, n-1) .
\end{aligned}
$$

We have proved Theorem 6.1 for $C_{n}^{(1)}$. 
6.4. $D_{n}^{(1)}(n \geq 4)$. Let us see the crystal structure of $\mathcal{U} D\left(\mathcal{V}\left(D_{n}^{(1)}\right)\right)=\left(\mathcal{B}\left(D_{n}^{(1)}\right),\left\{\tilde{e}_{i}\right\}\right.$, $\left.\left\{\mathrm{wt}_{i}\right\},\left\{\varepsilon_{i}\right\}\right)$. We have $\mathcal{B}\left(D_{n}^{(1)}\right)=\mathbb{Z}^{2 n-2}$ and for $x=\left(x_{1}, \cdots, x_{n}, \bar{x}_{n-2}, \cdots, \bar{x}_{1}\right) \in$ $\mathcal{B}\left(D_{n}^{(1)}\right)$, we have

$$
\begin{aligned}
& \tilde{e}_{0}(x)= \begin{cases}\left(x_{1}, x_{2}-1, \cdots, x_{n}-1, \cdots, \bar{x}_{2}-1, \bar{x}_{1}-1\right) & \text { if } x_{1}+\bar{x}_{1} \geq x_{2}+\bar{x}_{2}, \\
\left(x_{1}-1, x_{2}-1, \cdots, x_{n}-1, \cdots, \bar{x}_{2}-1, \bar{x}_{1}\right) & \text { if } x_{1}+\bar{x}_{1}<x_{2}+\bar{x}_{2},\end{cases} \\
& \tilde{e}_{i}(x)= \begin{cases}\left(x_{1}, \cdots, x_{i}+1, \cdots, \bar{x}_{i}, \cdots, \bar{x}_{1}\right) & \text { if } x_{i}+\bar{x}_{i} \geq x_{i+1}+\bar{x}_{i+1}, \\
\left(x_{1}, \cdots, x_{i}, \cdots, \bar{x}_{i}+1, \cdots, \bar{x}_{1}\right) & \text { if } x_{i}+\bar{x}_{i}<x_{i+1}+\bar{x}_{i+1},\end{cases} \\
& (i=1, \cdots, n-3), \\
& \tilde{e}_{n-2}(x)= \begin{cases}\left(x_{1}, \cdots, x_{n-2}+1, \cdots, \bar{x}_{n-2}, \cdots, \bar{x}_{1}\right) & \text { if } x_{n-2}+\bar{x}_{n-2} \geq x_{n-1}+x_{n}, \\
\left(x_{1}, \cdots, x_{n-2}, \cdots, \bar{x}_{n-2}+1, \cdots, \bar{x}_{1}\right) & \text { if } x_{n-2}+\bar{x}_{n-2}<x_{n-1}+x_{n},\end{cases} \\
& \tilde{e}_{n-1}(x)=\left(x_{1}, \cdots, x_{n-1}+1, x_{n}, \cdots, \bar{x}_{1}\right) \text {, } \\
& \tilde{e}_{n}(x)=\left(x_{1} \cdots, x_{n-1}, x_{n}+1, \cdots, \bar{x}_{1}\right) \text {, } \\
& \mathrm{wt}_{0}(x)=-\left(x_{2}+\bar{x}_{2}\right), \quad \mathrm{wt}_{1}(x)=2\left(x_{1}+\bar{x}_{1}\right)-\left(x_{2}+\bar{x}_{2}\right), \\
& \mathrm{wt}_{i}(x)=-\left(x_{i-1}+\bar{x}_{i-1}\right)+2\left(x_{i}+\bar{x}_{i}\right)-\left(x_{i+1}+\bar{x}_{i+1}\right) \quad(i=2, \cdots, n-3) \text {, } \\
& \mathrm{wt}_{n-2}(x)=-\left(x_{n-3}+\bar{x}_{n-3}\right)+2\left(x_{n-2}+\bar{x}_{n-2}\right)-\left(x_{n-1}+x_{n}\right) \text {, } \\
& \mathrm{wt}_{n-1}(x)=-\left(x_{n-2}+\bar{x}_{n-2}\right)+2\left(x_{n-1}+\bar{x}_{n-1}\right), \quad \mathrm{wt}_{n}(x)=2 x_{n}-\left(x_{n-2}+\bar{x}_{n-2}\right) \text {, } \\
& \varepsilon_{0}(x)=\max \left(x_{1}+\bar{x}_{1}, x_{2}+\bar{x}_{2}\right)-x_{1}=\bar{x}_{1}+\left(x_{2}+\bar{x}_{2}-x_{1}-\bar{x}_{1}\right)_{+}, \\
& \varepsilon_{1}(x)=\left(x_{2}+\bar{x}_{2}-x_{1}-\bar{x}_{1}\right)_{+}-x_{1}, \\
& \varepsilon_{i}(x)=x_{i-1}-x_{i}+\left(x_{i+1}+\bar{x}_{i+1}-x_{i}-\bar{x}_{i}\right)_{+}(i=2, \cdots, n-3), \\
& \varepsilon_{n-2}(x)=x_{n-3}-x_{n-2}+\left(x_{n-1}+x_{n}-x_{n-2}-\bar{x}_{n-2}\right)_{+} \text {, } \\
& \varepsilon_{n-1}(x)=x_{n-2}-x_{n-1}, \quad \varepsilon_{n}(x)=x_{n-2}-x_{n} .
\end{aligned}
$$

Then the following map gives an isomorphism of $D_{n}^{(1)}$-crystals:

$$
\begin{aligned}
\mu: \mathcal{B}\left(D_{n}^{(1)}\right) & \longrightarrow B_{\infty}\left(D_{n}^{(1)}\right) \\
\left(x_{1}, \cdots, x_{n-1}, x_{n}, \bar{x}_{n-2}, \cdots, \bar{x}_{1}\right) & \mapsto\left(b_{1}, \cdots, b_{n}, \bar{b}_{n-1}, \cdots, \bar{b}_{1}\right),
\end{aligned}
$$

where

$$
\begin{aligned}
& b_{1}=\bar{x}_{1}, \quad b_{i}=\bar{x}_{i}-\bar{x}_{i-1}(i=2, \cdots, n-2), \quad b_{n-1}=x_{n-1}-\bar{x}_{n-2}, \\
& b_{n}=x_{n}-x_{n-1}, \quad \bar{b}_{n-1}=x_{n-2}-x_{n}, \quad \bar{b}_{i}=x_{i-1}-x_{i}(i=2, \cdots, n-2), \\
& \bar{b}_{1}=-\sum_{i=1}^{n} b_{i}-\sum_{i=2}^{n-1} \bar{b}_{i}=-x_{1} .
\end{aligned}
$$

We have proved Theorem 6.1 for $D_{n}^{(1)}$.

6.5. $A_{2 n-1}^{(2)}(n \geq 3)$. The crystal structure of $\mathcal{U} D\left(\mathcal{V}\left(A_{2 n-1}^{(2)}\right)\right)=\left(\mathcal{B}\left(A_{2 n-1}^{(2)}\right),\left\{\tilde{e}_{i}\right\}\right.$, $\left.\left\{\mathrm{wt}_{i}\right\},\left\{\varepsilon_{i}\right\}\right)$ is given by: $\mathcal{B}\left(A_{2 n-1}^{(2)}\right)=\mathbb{Z}^{2 n-1}$ and for $x=\left(x_{1}, \cdots, x_{n}, \bar{x}_{n-1}, \cdots, \bar{x}_{1}\right) \in$ 
$\mathcal{B}\left(A_{2 n-1}^{(2)}\right)$, we have

$$
\begin{aligned}
& \tilde{e}_{0}(x)= \begin{cases}\left(x_{1}, x_{2}-1, \cdots, x_{n}-2, \cdots, \bar{x}_{2}-1, \bar{x}_{1}-1\right) & \text { if } x_{1}+\bar{x}_{1} \geq x_{2}+\bar{x}_{2}, \\
\left(x_{1}-1, x_{2}-1, \cdots, x_{n}-2, \cdots, \bar{x}_{2}-1, \bar{x}_{1}\right) & \text { if } x_{1}+\bar{x}_{1}<x_{2}+\bar{x}_{2},\end{cases} \\
& \tilde{e}_{i}(x)= \begin{cases}\left(x_{1}, \cdots, x_{i}+1, \cdots, \bar{x}_{i}, \cdots, \bar{x}_{1}\right) & \text { if } x_{i}+\bar{x}_{i} \geq x_{i+1}+\bar{x}_{i+1}, \\
\left(x_{1}, \cdots, x_{i}, \cdots, \bar{x}_{i}+1, \cdots, \bar{x}_{1}\right) & \text { if } x_{i}+\bar{x}_{i}<x_{i+1}+\bar{x}_{i+1},\end{cases} \\
& (i=1, \cdots, n-2) \text {, } \\
& \tilde{e}_{n-1}(x)= \begin{cases}\left(x_{1}, \cdots, x_{n-1}+1, x_{n}, \bar{x}_{n-1}, \cdots, \bar{x}_{1}\right) & \text { if } x_{n-1}+\bar{x}_{n-1} \geq x_{n}, \\
\left(x_{1}, \cdots, x_{n-1}, x_{n}, \bar{x}_{n-1}+1, \cdots, \bar{x}_{1}\right) & \text { if } x_{n-1}+\bar{x}_{n-1}<x_{n},\end{cases} \\
& \tilde{e}_{n}(x)=\left(x_{1}, \cdots, x_{n-1}, x_{n}+1, \bar{x}_{n-1}, \cdots, \bar{x}_{1}\right) \text {, } \\
& \mathrm{wt}_{0}(x)=-\left(x_{2}+\bar{x}_{2}\right), \quad \mathrm{wt}_{1}(x)=2\left(x_{1}+\bar{x}_{1}\right)-\left(x_{2}+\bar{x}_{2}\right), \\
& \mathrm{wt}_{i}(x)=-\left(x_{i-1}+\bar{x}_{i-1}\right)+2\left(x_{i}+\bar{x}_{i}\right)-\left(x_{i+1}+\bar{x}_{i+1}\right) \quad(i=2, \cdots, n-2) \text {, } \\
& \mathrm{wt}_{n-1}(x)=-\left(x_{n-2}+\bar{x}_{n-2}\right)+2\left(x_{n-1}+\bar{x}_{n-1}\right)-x_{n} \text {, } \\
& \mathrm{wt}_{n}(x)=2 x_{n}-2\left(x_{n-1}+\bar{x}_{n-1}\right) \text {, } \\
& \varepsilon_{0}(x)=\max \left(x_{1}+\bar{x}_{1}, x_{2}+\bar{x}_{2}\right)-x_{1}=\bar{x}_{1}+\left(x_{2}+\bar{x}_{2}-x_{1}-\bar{x}_{1}\right)_{+}, \\
& \varepsilon_{1}(x)=\left(x_{2}+\bar{x}_{2}-x_{1}-\bar{x}_{1}\right)_{+}-x_{1}, \\
& \varepsilon_{i}(x)=x_{i-1}-x_{i}+\left(x_{i+1}+\bar{x}_{i+1}-x_{i}-\bar{x}_{i}\right)_{+}(i=2, \cdots, n-2) \text {, } \\
& \varepsilon_{n-1}(x)=x_{n-2}-x_{n-1}+\left(x_{n}-x_{n-1}-\bar{x}_{n-1}\right)_{+}, \quad \varepsilon_{n}(x)=2 x_{n-1}-x_{n} .
\end{aligned}
$$

Then the following map gives an isomorphism of $B_{n}^{(1)}$-crystals:

$$
\begin{aligned}
\mu: \mathcal{B}\left(A_{2 n-1}^{(2)}\right) & \longrightarrow B_{\infty}\left(B_{n}^{(1)}\right) \\
\left(x_{1}, \cdots, x_{n}, \cdots, \bar{x}_{1}\right) & \mapsto \quad\left(b_{1}, \cdots, b_{n}, \bar{b}_{n}, \cdots, \bar{b}_{1}\right),
\end{aligned}
$$

where

$$
\begin{aligned}
& b_{1}=\bar{x}_{1}, \quad b_{i}=\bar{x}_{i}-\bar{x}_{i-1}(i=2, \cdots, n-1), b_{n}=\frac{1}{2} x_{n}-\bar{x}_{n-1}, \\
& \bar{b}_{n}=x_{n-1}-\frac{1}{2} x_{n}, \quad \bar{b}_{i}=x_{i-1}-x_{i}(i=2, \cdots, n-1), \bar{b}_{1}=-x_{1} .
\end{aligned}
$$

We have proved Theorem 6.1 for $A_{2 n-1}^{(2)}$.

6.6. $D_{n+1}^{(2)}(n \geq 2)$. Let us see the crystal structure of $\mathcal{U} D\left(\mathcal{V}\left(D_{n+1}^{(2)}\right)\right)=\left(\mathcal{B}\left(D_{n+1}^{(2)}\right)\right.$ $\left.\left\{\tilde{e}_{i}\right\},\left\{\mathrm{wt}_{i}\right\},\left\{\varepsilon_{i}\right\}\right)$. We have $\mathcal{B}\left(D_{n+1}^{(2)}\right)=\mathbb{Z}^{2 n}$ and for $x=\left(x_{0}, x_{1}, \cdots, x_{n}, \bar{x}_{n-1}, \cdots, \bar{x}_{1}\right)$ $\in \mathcal{B}\left(D_{n+1}^{(2)}\right)$, we have

$$
\tilde{e}_{0}(x)=\left\{\begin{array}{lr}
\left(x_{0}+1, x_{1}, \cdots, x_{n}, \bar{x}_{n-1}, \cdots, \bar{x}_{2}, \bar{x}_{1}\right) \quad \text { if } 2 x_{0} \geq x_{1}+\bar{x}_{1}, \\
\left(x_{0}, x_{1}-1, \cdots, x_{n}-1, \bar{x}_{n-1}-1, \cdots, \bar{x}_{1}-1\right) & \text { if } 2 x_{0}+1=x_{1}+\bar{x}_{1}, \\
\left(x_{0}-1, \cdots, x_{n-1}-2, x_{n}-2, \bar{x}_{n-1}-2, \cdots, \bar{x}_{2}-2, \bar{x}_{1}-2\right) & \text { if } 2 x_{0}+1<x_{1}+\bar{x}_{1},
\end{array}\right.
$$


where we use the formula

$$
\begin{aligned}
& \max (2+x, y)-\max (x, y)= \begin{cases}2 & x \geq y \\
1 & x+1=y \quad(x, y \in \mathbb{Z}), \\
0 & x+1<y\end{cases} \\
& \tilde{e}_{i}(x)= \begin{cases}\left(x_{1}, \cdots, x_{i}+1, \cdots, \bar{x}_{i}, \cdots, \bar{x}_{1}\right) & \text { if } x_{i}+\bar{x}_{i} \geq x_{i+1}+\bar{x}_{i+1}, \\
\left(x_{1}, \cdots, x_{i}, \cdots, \bar{x}_{i}+1, \cdots, \bar{x}_{1}\right) & \text { if } x_{i}+\bar{x}_{i}<x_{i+1}+\bar{x}_{i+1},\end{cases} \\
& (i=1, \cdots, n-2) \text {, } \\
& \tilde{e}_{n-1}(x)= \begin{cases}\left(x_{1}, \cdots, x_{n-1}+1, x_{n}, \bar{x}_{n-1}, \cdots, \bar{x}_{1}\right) & \text { if } x_{n-1}+\bar{x}_{n-1} \geq 2 x_{n}, \\
\left(x_{1}, \cdots, x_{n-1}, x_{n}, \bar{x}_{n-1}+1, \cdots, \bar{x}_{1}\right) & \text { if } x_{n-1}+\bar{x}_{n-1}<2 x_{n},\end{cases} \\
& \tilde{e}_{n}(x)=\left(x_{1}, \cdots, x_{n-1}, x_{n}+1, \bar{x}_{n-1}, \cdots, \bar{x}_{1}\right) \text {, } \\
& \mathrm{wt}_{0}(x)=2 x_{0}-\left(x_{1}+\bar{x}_{1}\right), \quad \mathrm{wt}_{1}(x)=2\left(x_{1}+\bar{x}_{1}\right)-\left(2 x_{0}+x_{2}+\bar{x}_{2}\right), \\
& \mathrm{wt}_{i}(x)=-\left(x_{i-1}+\bar{x}_{i-1}\right)+2\left(x_{i}+\bar{x}_{i}\right)-\left(x_{i+1}+\bar{x}_{i+1}\right) \quad(i=2, \cdots, n-2) \text {, } \\
& \mathrm{wt}_{n-1}(x)=-\left(x_{n-2}+\bar{x}_{n-2}\right)+2\left(x_{n-1}+\bar{x}_{n-1}\right)-2 x_{n} \text {, } \\
& \mathrm{wt}_{n}(x)=2 x_{n}-\left(x_{n-1}+\bar{x}_{n-1}\right) \text {, } \\
& \varepsilon_{0}(x)=-x_{0}+\left(x_{1}+\bar{x}_{1}-2 x_{0}\right)_{+}, \quad \varepsilon_{1}(x)=2 x_{0}-x_{1}+\left(x_{2}+\bar{x}_{2}-x_{1}-\bar{x}_{1}\right)_{+}, \\
& \varepsilon_{i}(x)=x_{i-1}-x_{i}+\left(x_{i+1}+\bar{x}_{i+1}-x_{i}-\bar{x}_{i}\right)_{+}(i=2, \cdots, n-2), \\
& \varepsilon_{n-1}(x)=x_{n-2}-x_{n-1}+\left(2 x_{n}-x_{n-1}-\bar{x}_{n-1}\right)_{+}, \quad \varepsilon_{n}(x)=x_{n-1}-x_{n} .
\end{aligned}
$$

Then the following map gives an isomorphism of $C_{n}^{(1)}$-crystals:

$$
\begin{aligned}
\mu: \mathcal{B}\left(D_{n+1}^{(2)}\right) & \longrightarrow B_{\infty}\left(C_{n}^{(1)}\right) \\
\left(x_{0}, x_{1}, \cdots, x_{n}, \cdots, \bar{x}_{1}\right) & \mapsto\left(b_{1}, \cdots, b_{n}, \bar{b}_{n}, \cdots, \bar{b}_{1}\right),
\end{aligned}
$$

where

$b_{1}=\bar{x}_{1}, b_{i}=\bar{x}_{i}-\bar{x}_{i-1}(i=2, \cdots, n), \bar{b}_{i}=x_{i-1}-x_{i}(i=2, \cdots, n), \bar{b}_{1}=2 x_{0}-x_{1}$.

We have proved Theorem 6.1 for $D_{n+1}^{(2)}$.

6.7. $A_{2 n}^{(2) \dagger}(n \geq 2)$. Let $\mathcal{V}\left(A_{2 n}^{(2) \dagger}\right)$ be the affine $A_{2 n}^{(2) \dagger}$-geometric crystal as in $\$ 5.9$, We shall see that the crystal structure of $\mathcal{U} D\left(\mathcal{V}\left(A_{2 n}^{(2) \dagger}\right)\right)=\left(\mathcal{B}\left(A_{2 n}^{(2) \dagger}\right),\left\{\tilde{e}_{i}\right\}\right.$, $\left.\left\{\mathrm{wt}_{i}\right\},\left\{\varepsilon_{i}\right\}\right)$ is given by: $\mathcal{B}\left(A_{2 n}^{(2) \dagger}\right)=\mathbb{Z}^{2 n}$ and for $x=\left(x_{0}, x_{1}, \cdots, x_{n}, \bar{x}_{n-1}, \cdots, \bar{x}_{1}\right) \in$ $\mathcal{B}\left(A_{2 n}^{(2) \dagger}\right)$, we have

$$
\begin{aligned}
& \tilde{e}_{0}(x)=\left\{\begin{array}{cc}
\left(x_{0}+1, x_{1}, \cdots, x_{n}, \bar{x}_{n-1}, \cdots, \bar{x}_{2}, \bar{x}_{1}\right) & \text { if } x_{0} \geq x_{1}+\bar{x}_{1}, \\
\left(x_{0}-1, x_{1}-1 \cdots, x_{n-1}-1, x_{n}-1, \bar{x}_{n-1}-1, \cdots, \bar{x}_{1}-1\right) & \text { if } x_{0}<x_{1}+\bar{x}_{1},
\end{array}\right. \\
& \tilde{e}_{i}(x)=\left\{\begin{array}{rr}
\left(x_{1}, \cdots, x_{i}+1, \cdots, \bar{x}_{i}, \cdots, \bar{x}_{1}\right) & \text { if } x_{i}+\bar{x}_{i} \geq x_{i+1}+\bar{x}_{i+1}, \\
\left(x_{1}, \cdots, x_{i}, \cdots, \bar{x}_{i}+1, \cdots, \bar{x}_{1}\right) & \text { if } x_{i}+\bar{x}_{i}<x_{i+1}+\bar{x}_{i+1}, \\
(i=1, \cdots, n-2),
\end{array}\right. \\
& \tilde{e}_{n-1}(x)= \begin{cases}\left(x_{1}, \cdots, x_{n-1}+1, x_{n}, \bar{x}_{n-1}, \cdots, \bar{x}_{1}\right) & \text { if } x_{n-1}+\bar{x}_{n-1} \geq 2 x_{n}, \\
\left(x_{1}, \cdots, x_{n-1}, x_{n}, \bar{x}_{n-1}+1, \cdots, \bar{x}_{1}\right) & \text { if } x_{n-1}+\bar{x}_{n-1}<2 x_{n},\end{cases} \\
& \tilde{e}_{n}(x)=\left(x_{1}, \cdots, x_{n-1}, x_{n}+1, \bar{x}_{n-1}, \cdots, \bar{x}_{1}\right),
\end{aligned}
$$




$$
\begin{aligned}
& \mathrm{wt}_{0}(x)=2 x_{0}-2\left(x_{1}+\bar{x}_{1}\right), \quad \mathrm{wt}_{1}(x)=2\left(x_{1}+\bar{x}_{1}\right)-\left(x_{0}+x_{2}+\bar{x}_{2}\right), \\
& \mathrm{wt}_{i}(x)=-\left(x_{i-1}+\bar{x}_{i-1}\right)+2\left(x_{i}+\bar{x}_{i}\right)-\left(x_{i+1}+\bar{x}_{i+1}\right) \quad(i=2, \cdots, n-2), \\
& \mathrm{wt}_{n-1}(x)=-\left(x_{n-2}+\bar{x}_{n-2}\right)+2\left(x_{n-1}+\bar{x}_{n-1}\right)-2 x_{n}, \\
& \mathrm{wt}_{n}(x)=2 x_{n}-\left(x_{n-1}+\bar{x}_{n-1}\right), \\
& \varepsilon_{0}(x)=-x_{0}+2\left(x_{1}+\bar{x}_{1}-x_{0}\right)_{+}, \\
& \varepsilon_{i}(x)=x_{i-1}-x_{i}+\left(x_{i+1}+\bar{x}_{i+1}-x_{i}-\bar{x}_{i}\right)_{+}(i=1, \cdots, n-2), \\
& \varepsilon_{n-1}(x)=x_{n-2}-x_{n-1}+\left(2 x_{n}-x_{n-1}-\bar{x}_{n-1}\right)_{+}, \quad \varepsilon_{n}(x)=x_{n-1}-x_{n} .
\end{aligned}
$$

Then the following map gives an isomorphism of $A_{2 n}^{(2)}$-crystals:

$$
\begin{aligned}
\mu: \mathcal{B}\left(A_{2 n}^{(2) \dagger}\right) & \longrightarrow B_{\infty}\left(A_{2 n}^{(2)}\right) \\
\left(x_{0}, x_{1}, \cdots, x_{n}, \cdots, \bar{x}_{1}\right) & \mapsto\left(b_{1}, \cdots, b_{n}, \bar{b}_{n}, \cdots, \bar{b}_{1}\right),
\end{aligned}
$$

where

$$
\begin{aligned}
b_{1}=\bar{x}_{1}, & b_{i}=\bar{x}_{i}-\bar{x}_{i-1}(i=2, \cdots, n-1), b_{n}=x_{n}-\bar{x}_{n-1}, \\
\bar{b}_{i} & =x_{i-1}-x_{i}(i=1, \cdots, n) .
\end{aligned}
$$

We have proved Theorem 6.1 for $A_{2 n}^{(2) \dagger}$.

\section{REFERENCES}

[1] Berenstein, A. and Kazhdan, D., Geometric and unipotent crystals, GAFA 2000 (Tel Aviv, 1999), Geom Funct. Anal. Special Volume, Part I, (2000) 188-236. MR 1826254|(2003b:17013)

[2] Hatayama, G., Kuniba, A., Okado, M., Takagi, T. and Yamada, Y., Remarks on fermionic formula, Contemp. Math. 248 (1999) 243-291. MR1745263 (2001m:81129)

[3] Hatayama, G., Kuniba, A., Okado, M., Takagi, T. and Tsuboi, Z., Paths, crystals and fermionic formulae, in "MathPhys Odyssey 2001-Integrable Models and Beyond In Honor of Barry M.McCoy", edited by M. Kashiwara and T. Miwa, Birkhäuser (2002) 205-272. MR1903978 (2003e:17020)

[4] Kac, V.G., Infinite Dimensional Lie Algebras, Cambridge Univ. Press, 3rd edition (1990). MR1104219 (92k:17038)

[5] Kac, V.G. and Peterson, D.H., Defining relations of certain infinite-dimensional groups, in "Arithmetic and Geometry" (Artin M., Tate J., eds), Birkhäuser, Boston-Basel-Stuttgart, (1983) 141-166. MR717610 (86b:17010)

[6] Kang, S.-J., Kashiwara, M. and Misra, K.C., Crystal bases of Verma modules for quantum affine Lie algebras, Compositio Mathematica 92 (1994) 299-325. MR1286129 (95h:17016)

[7] Kang, S.-J., Kashiwara, M., Misra, K.C., Miwa, T., Nakashima, T. and Nakayashiki, A., Affine crystals and vertex models, Int. J. Mod. Phys., A7 Suppl. 1A (1992) 449-484. MR1187560 (94a:17008)

[8] _ Perfect crystals of quantum affine Lie algebras, Duke Math. J., 68(3), (1992) 499607. MR.1194953 (94j:17013)

[9] Kashiwara, M., On level-zero representation of quantized affine algebras, Duke Math. J., 112 (2002) 499-525. MR 1890649 (2002m:17013)

[10] L Level zero fundamental representations over quantized affine algebras and Demazure modules, Publ. Res. Inst. Math. Sci. 41 (2005) no. 1, 223-250. MR2115972 (2005i:17021)

[11] Kumar, S., Kac-Moody Groups, their Flag Varieties and Representation Theory, Progress in Mathematics 204, Birkhäuser Boston, (2002). MR 1923198 (2003k:22022)

[12] Kuniba, A., Misra, K.C., Okado, M., Takagi T. and Uchiyama J., Crystals for Demazure modules of classical affine Lie algebras, J. of Alg. 208 (1998) 185-215. MR.1643999 (99h:17008)

[13] Kuniba, A., Okado, M., Takagi, T. and Yamada, Y., Geometric crystals and tropical $\mathcal{R}$ for $D_{n}^{(1)}$, IMRN, no.48, (2003) 2565-2620. MR2013509 (2004h:17016)

[14] Nakashima, T., Geometric crystals on Schubert varieties, Journal of Geometry and Physics, 53 (2), (2005) 197-225. MR2110832 (2006d:17016) 
[15] - Geometric crystals on unipotent groups and generalized Young tableaux, Journal of Algebra, 293 (2005) No.1, 65-88. MR.2173966 (2006j:20064)

[16] Peterson, D.H. and Kac, V.G., Infinite flag varieties and conjugacy theorems, Proc. Nat. Acad. Sci. USA, 80, (1983) 1778-1782. MR699439 (84g:17017)

Research Institute for Mathematical Sciences, Kyoto University, Kitashiwakawa, SAKYO-KU, КYOTO 606, JAPAN

E-mail address: masaki@kurims.kyoto-u.ac.jp

Department of Mathematics, Sophia University, Kioicho 7-1, Chiyoda-Ku, Tokyo $102-$ 8554, JAPAN

E-mail address: toshiki@mm.sophia.ac.jp

Department of Mathematical Science, Graduate School of Engineering Science, Osaka University, TOYONAKa, Osaka 560-8531, JAPAN

E-mail address: okado@sigmath.es.osaka-u.ac.jp 